\title{
On the Bennelongia barangaroo lineage (Crustacea, Ostracoda) in Western Australia, with the description of seven new species
}

\author{
Koen MARTENS ${ }^{1,2,5}$, Stuart HALSE ${ }^{3,6}$ \& Isa SCHÖN S $^{1,4,7}$ \\ ${ }^{1}$ Royal Belgian Institute of Natural Sciences, Freshwater Biology, Vautierstraat 29, \\ B-1000 Brussels, Belgium. Corresponding author: darwinula@gmail.com \\ ${ }^{2}$ University of Ghent, Department of Biology, K.L. Ledeganckstraat 35, B-9000 Gent, Belgium. \\ ${ }^{3}$ Bennelongia Environmental Consultants, 5 Bishop Street, Jolimont WA 6014, Australia. \\ ${ }^{4}$ University of Hasselt, Research Group Zoology, Agoralaan Building D, \\ B-3590 Diepenbeek, Belgium \\ ${ }^{5}$ urn:Isid:zoobank.org:author:9272757B-A9E5-4C94-B28D-F5EFF32AADC7 \\ ${ }^{6}$ urn:lsid:zoobank.org:author:676014BF-5979-49EC-BC8F-811214170111 \\ 7 urn:1sid:zoobank.org:author:94232F10-7092-4E90-9071-64C4FDABE691
}

\begin{abstract}
The ostracod genus Bennelongia De Deckker \& McKenzie, 1981 is endemic to Australia and New Zealand. Extensive sampling in Western Australia (WA) revealed a high specific and largely undescribed diversity. Here, we describe seven new species belonging to the $B$. barangaroo lineage: B. timmsi sp. nov., B. gnamma sp. nov., B. hirsuta sp. nov., B. ivanae sp. nov., B. mcraeae sp. nov., B. scanloni sp. nov. and B. calei sp. nov., and confirm the presence of an additional species, $B$. dedeckkeri, in WA. For five of these eight species, we could construct molecular phylogenies and parsimonious networks based on COI sequences. We also tested for cryptic diversity and specific status of clusters with a statistical method based on the evolutionary genetic species concept, namely Birky's 4 theta rule. The analyses support the existence of these five species and a further three cryptic species in the WA $B$. barangaroo lineage. The molecular evidence was particularly relevant because most species described herein have very similar morphologies and can be distinguished from each other only by the shape, size and position of the antero-ventral lapel on the right valve, and, in sexual populations, by the small differences in shape of the hemipenes and the prehensile palps in males. Four species of the WA B. barangaroo lineage occur in small temporary rock pools (gnammas) on rocky outcrops. The other four species are mainly found in soft bottomed seasonal water bodies. One of the latter species, B. scanloni sp. nov., occurs in both claypans and deeper rock pools (pit gnammas). All species, except for $B$. dedeckkeri, originally described from Queensland, have quite clearly delimited distributions in WA. With the seven new species described here, the genus Bennelongia now comprises 25 nominal species but several more await formal description.
\end{abstract}

Keywords. Taxonomy, evolution, cryptic species, biodiversity, Western Australia.

Martens K., Halse S. \& Schön I. 2013, On the Bennelongia barangaroo lineage (Crustacea, Ostracoda) in Western Australia, with the description of seven new species. European Journal of Taxonomy 66: 1-59. http://dx.doi. org/10.5852/ejt.2013.66 


\section{Introduction}

The ostracod genus Bennelongia is endemic to Australia and New Zealand. Extensive sampling in Western Australia (WA) revealed a high specific and largely undescribed diversity of Bennelongia (Halse 2002), leading to the taxonomic research reported here. The present paper is the fourth in a recent series of taxonomic contributions on Australian Bennelongia. Martens et al. (2012) described nine new species in three different lineages within the genus, all based on collections from WA. Shearn et al. (2012) redescribed several extant species and described three new species, all from Eastern Australia (mostly from Queensland). In addition, De Deckker \& Martens (2013) described the unusually strong morphological changes in valve morphology during the last 3-4 ontogenetic stages in several Bennelongia-species, and showed that these changes can be different between the various lineages within the genus. The first two papers, together with the earlier work by De Deckker (1981a,b, 1982) and De Deckker \& McKenzie (1981), brought the number of nominal species in the genus to 18 , but both recent papers also recognised that some additional cryptic species exist, as determined by molecular methods.

Here, we describe seven new species of Bennelongia from WA and report on the occurrence of an additional described species in WA, namely $B$. dedeckkeri Shearn et al., 2012. All of these eight species belong to the $B$. barangaroo lineage within the genus and for five of the species, their specific status is confirmed with molecular methods. Two of the new nominal species furthermore comprise several, sometimes sympatric, cryptic species that could not be recognised using either valve or soft part morphologies.

\section{Material and methods}

\section{Collections}

Ostracods were collected from pans, lakes and rock pools with a hand net with mesh size of $250 \mu \mathrm{m}$ during several field trips (see below). Material for morphological analyses originated from both these 'new' collections and from earlier collections from all over WA, mostly collected by SH and preserved in a collection housed at the Department of Environment and Conservation, now DPaW (Woodvale, Perth). The molecular analyses were successful only with newly collected material, using either living specimens or specimens sorted directly in the field and preserved in 100\% ethanol. Consequently, molecular analyses were limited to five of the eight species (four of the seven new ones). Locations of populations used for the present paper are indicated on the map in Fig. 1. Type material of the new species is deposited in the Western Australian Museum, Perth, WA (WAM numbers) and in the Ostracod Collection of the Royal Belgian Institute of Natural Sciences, Brussels, Belgium (OC numbers) (see Table 1).

\section{Morphological analyses}

Ostracods were dissected with valves stored dry in micropalaeontological slides and soft parts in glycerine in sealed slides. Drawings of soft parts were made with a camera lucida with a compound microscope (Leica, DM 2500 at Bennelongia Environmental Consultants, Perth). Valves were illustrated and measured using scanning electron microscopy (Philips XL30 SEM at RBINS, Brussels).

\section{Molecular analysis}

The Qiagen Blood and Tissue extraction kit was used following the manufacturer's protocol to extract DNA from 99 ostracods representing four nominal and 2 cryptic species of the Bennelongia barangaroo lineage. Universal PCR primers (Folmer et al. 1994) were applied to amplify part of the mitochondrial COI region in a $\mathrm{T}$ personal Thermoblock (Biometra) with the following conditions: $25 \mu \mathrm{l}$ volumes of the HotStar Master Mix (Qiagen; $1.5 \mathrm{mM} \mathrm{MgCl} 2,0.1 \mu \mathrm{M}$ primer, $200 \mu \mathrm{M}$ dNTP, Tris $\cdot \mathrm{Cl}, \mathrm{KCl},\left(\mathrm{NH}_{4}\right)_{2} \mathrm{SO}_{4}$, $1.25 \mathrm{U} \mathrm{Taq}$ ) and $15 \mathrm{~min}$ at $95^{\circ} \mathrm{C}, 40$ cycles of $1 \mathrm{~min}$ at $95^{\circ} \mathrm{C}, 1 \mathrm{~min}$ at $44^{\circ} \mathrm{C}, 1 \mathrm{~min}$ at $72^{\circ} \mathrm{C}$, followed 
by a final extension step for $10 \mathrm{~min}$ at $72^{\circ} \mathrm{C}$. Agarose gel electrophoresis and staining of gels with Gelred $^{\mathrm{TM}}$ was carried out to check for successful PCR amplifications. PCR products were cleaned with the GFX ${ }^{\mathrm{TM}}$ PCR DNA and gel band purification kit (GE Healthcare) according to the manufacturer's protocol and sequenced in both directions with the universal COI primers and the Big Dye kit (ABI) on an $\mathrm{ABI}$ 3130X following the manufacturer's protocol.

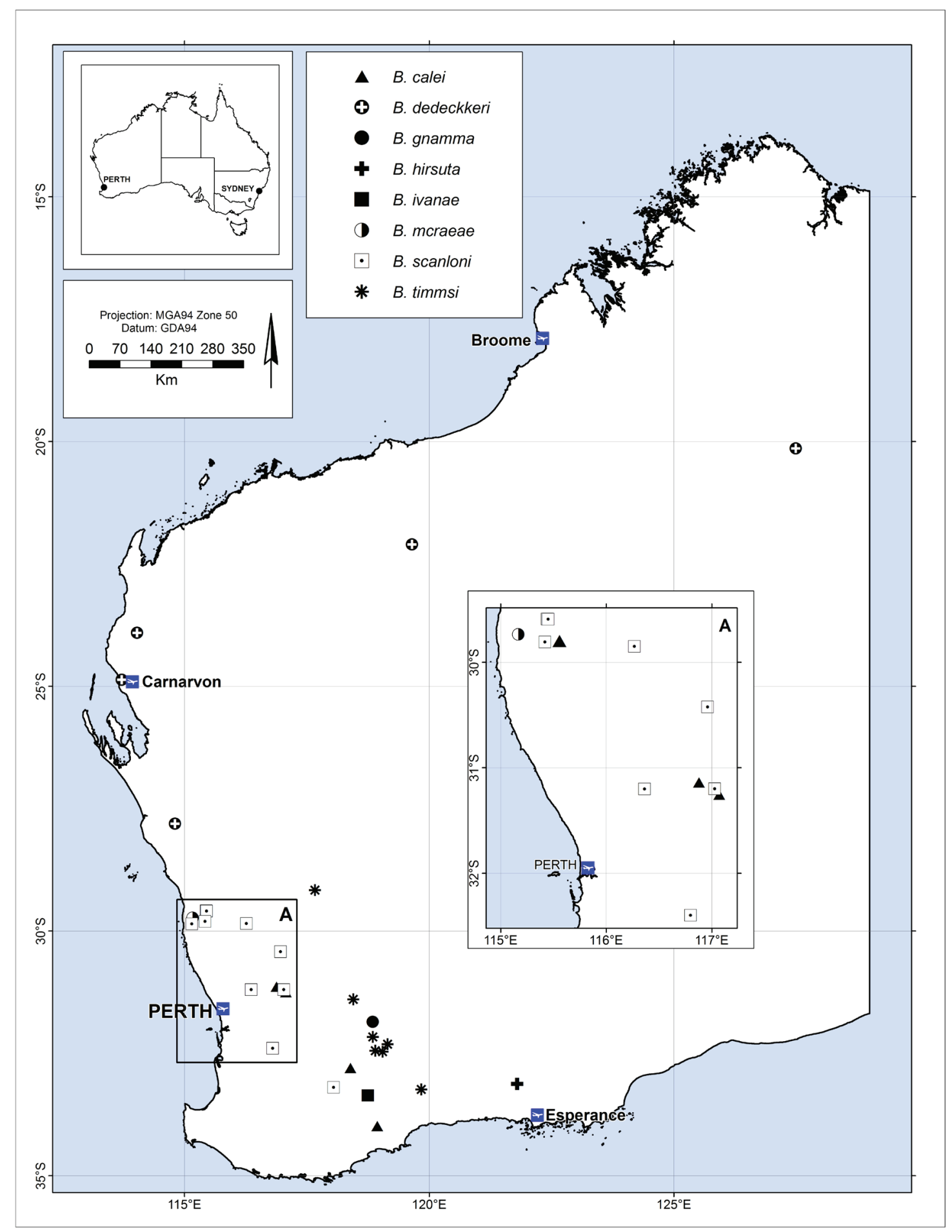

Fig. 1. Map of Western Australia with localities of Bennelongia - species described in the present paper. 
No fresh (living) material of three of the seven new nominal species in the barangaroo lineage could be obtained (namely B. gnamma sp. nov., B. hirsuta sp. nov. and B. mcraeae sp. nov.); these species are not represented in the molecular phylogenetic tree and network.

\section{Analyses of sequence data}

Sequence chromatograms were visualised with BioEdit (Hall 2007). For each individual, the forward and reverse strand were aligned with ClustalX (Larkin et al. 2007), subsequently checked by eye for ambiguities, corrected and finally trimmed to obtain sequences of equal lengths. BLAST searches (Altschul et al. 1990) were used to confirm the identity of the obtained sequences in Genbank. We applied jModeltest 2.1.1 (Darriba et al. 2012) to identify the optimal model of molecular COI evolution using 88 or 24 models and the AICc criterion. Two different methods were used for phylogenetic reconstructions, Bayesian Inference (BI) in Mr Bayes 3.2 (Ronquist et al. 2011; with 4 million generations, sampling every $100^{\text {th }}$ generation, a burn-in of $25 \%$ and the parameters identified by jModeltest for 24 different models) and the Maximum-Likelihood method in PhyML (Guindon \& Gascuel 2003; with 1000 bootstrap replicates and the parameters of jModeltest for all 88 models). We also constructed a parsimonious network at the 95\% probability limit with TCS 1.21 (Clement et al. 2000) to connect different sequences (or haplotypes) and to illustrate genetic diversities and genetic relationships within and between populations. Selected sequences of all species have been submitted to Genbank (accession numbers KF724982-KF725015; see Table 1).

\section{Testing for cryptic diversity}

We applied the 4 theta rule (Birky et al. 2010; Birky 2011) based on the evolutionary genetic species concept (Birky \& Barraclough 2009) to identify species boundaries and unravel cryptic diversities. This technique has been successfully used in bdelloid rotifers (Fontaneo et al. 2007, 2009; Birky \& Barraclough 2009; Birky et al. 2011), asexual ostracods (Schön et al. 2012) including other Bennelongia ostracod species (Martens et al. 2012; Shearn et al. 2012), and a wide range of asexual prokaryotes (Birky et al. 2010).

We used the COI phylogenetic tree to identify statistically supported clades, which could potentially be different species according to the evolutionary genetic species concept. We then estimated sequence diversities within and between these phylogenetic clades with MEGA 5.0 (Tamura et al. 2011) using the number of differences (p) and the Tamura-3 parameter model with gamma distribution (allowing for multiple hits, different transition and transversion rates and GC bias) and 1000 bootstrap replicates. Following the procedure by Birky et al. (2010), sequence diversities were subsequently corrected for sample size and sequence lengths. In order to fulfill the criteria of the 4 theta rule, the sequence diversity between two sister clades must be at least 4 to 4.3 times larger than within the two clades, depending on the number of samples per clade (Birky et al. 2010).

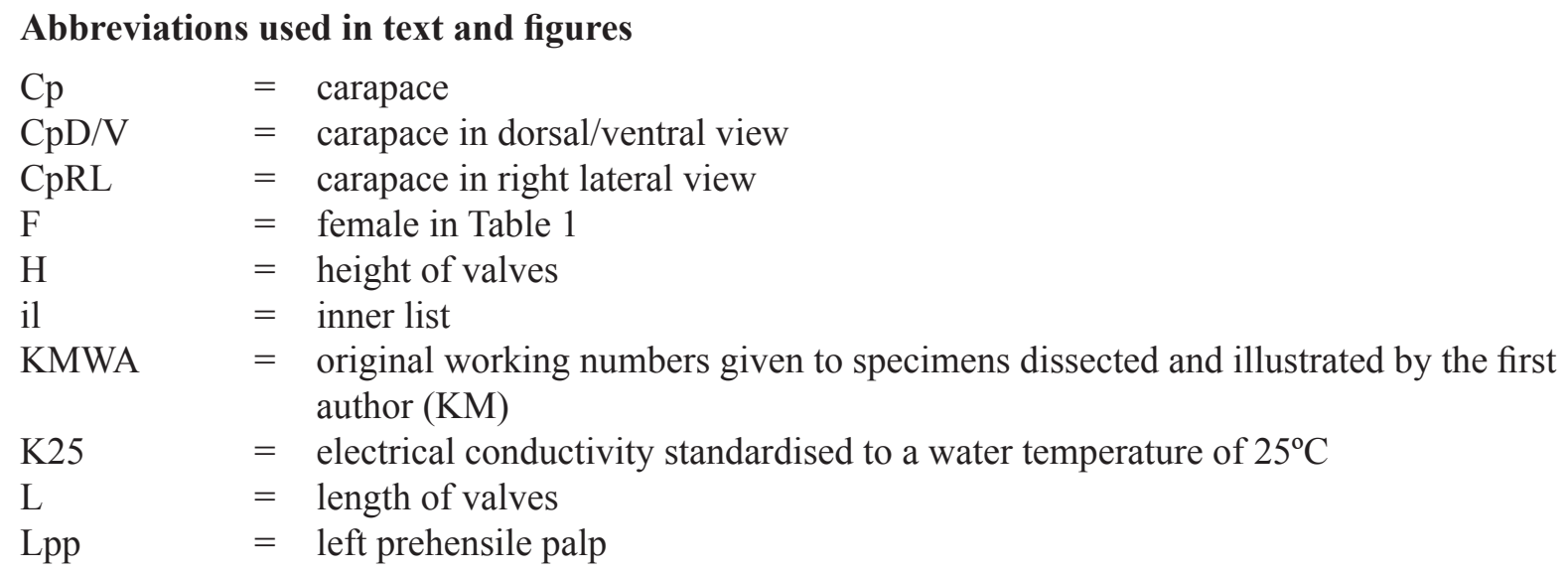


MARTENS K., HALSE S. \& SCHÖN I., The Bennelongia barangaroo lineage in Western Australia

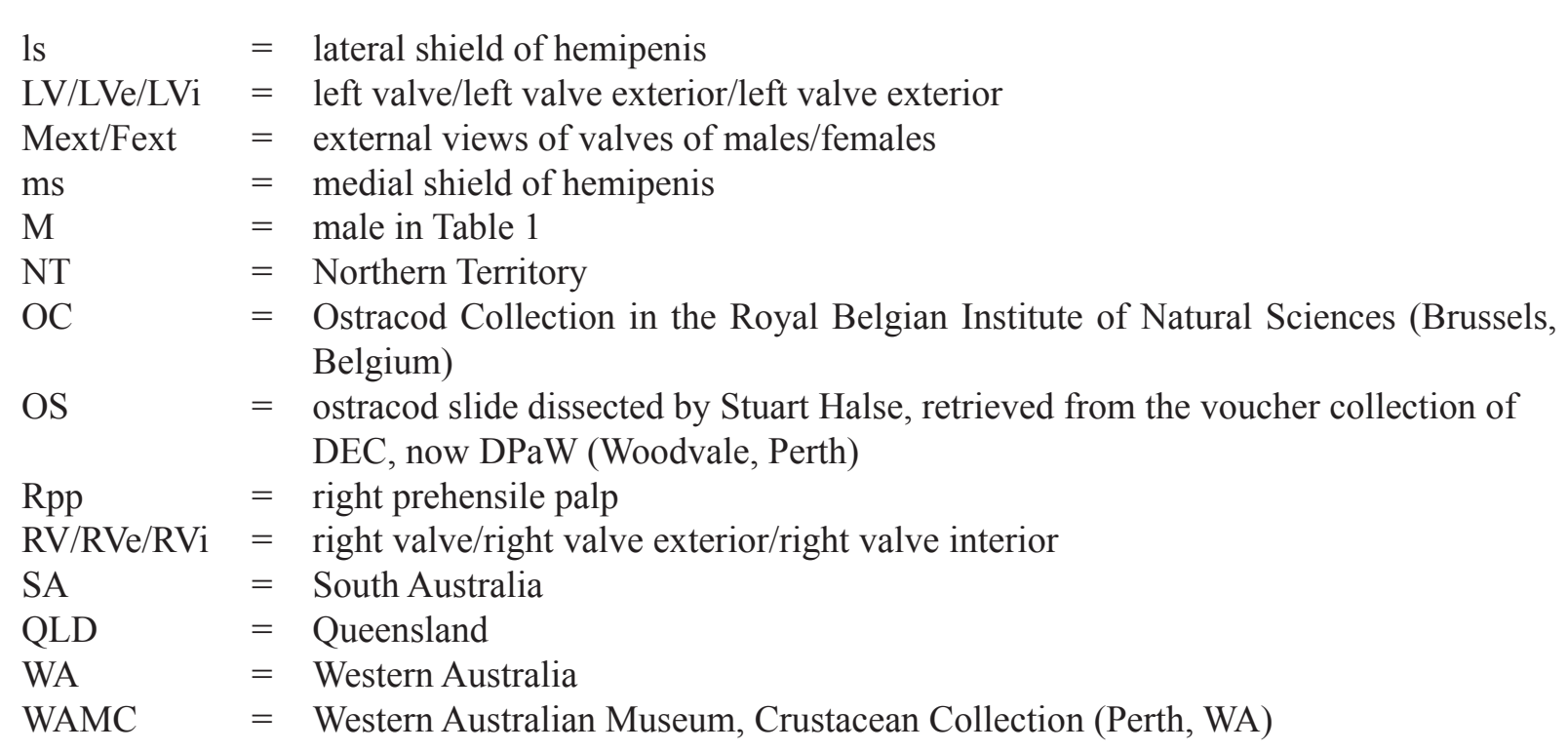

Specimens in bold in Table 1 are the holotypes of the species.

Chaetotaxy of the limbs follows the model proposed by Broodbakker \& Danielopol (1982), revised for A2 by Martens (1987). The higher taxonomy of the Ostracoda follows the synopsis by Horne et al. (2002).

\section{Results}

\section{Results of molecular screening}

We obtained 680 nucleotide-long sequences for part of the mitochondrial COI region for all 99 ostracods subjected to DNA extraction. $\mathrm{j}$ Modeltest selected the TPM1 uf $+\mathrm{I}+\mathrm{G}$ model with the following parameters among 88 models: freq $\mathrm{A}=0.33$; freqC $=0.19$; freqG $=0.13$; freq $=0.35 ;[\mathrm{AC}]=1.00 ;[\mathrm{AG}]=24.84$, $[\mathrm{AT}]=2.91 ;[\mathrm{CG}]=2.91 ;[\mathrm{CT}]=24.84 ;[\mathrm{GT}]=1.00 ; \mathrm{p}-\mathrm{inv}=0.56$; gamma shape $=1.26$. For Bayesian Inference, the $\mathrm{HKY}+\mathrm{I}+\mathrm{G}$ model was selected among 24 models.

The phylogenetic tree (Fig. 2) had a similar topology with ML and BI methods for tree construction. The two clades F1 and F2 (belonging to $B$. ivanae sp. nov. and $B$. sp. nov. F2 respectively) group together with high statistical support. They form the most basal branch and are separated from all other Bennelongia specimens. Within the phylogenetic cluster containing the other species, $B$. dedeckkeri forms the most basal branch, followed by clade B1 morphologically forming the new species $B$. calei sp. nov. The remaining specimens can be divided into three subgroups with strong statistical support for the tips but less support for the basal nodes of the subgroups themselves. Subgroup one consists of clade B2 as well as E1 and TST, which form sister clades and all belong morphologically to the new species $B$. scanloni sp. nov. The second subgroup is composed of clade $\mathrm{E} 2$, which is morphospecies $B$. sp. nov. E2 and sister clades A3 and A1, while the third subgroup contains clades A4, A2 and A5. According to their morphology, all A clades belong to the new species B. timmsi sp. nov.

We then used the topology of the phylogenetic tree (Fig. 2) to test whether clades phylogenetically closest to each other are in fact different genetic species by applying the 4 theta rule (see Table 2). Most sister clades represent different genetic species according to the criteria of the 4 theta rule (Birky et al. 2010), with the majority of these genetic species matching the morphologically identified species. This is the case for sister clades F1 and F2 representing B. ivanae sp. nov. and $B$. sp. nov. F2, respectively, and for $\mathrm{B} 1$ corresponding to $B$. calei sp. nov.. Likewise, morphology and genetics are concordant for the described species $B$. dedeckkeri and clade E2 (B. spec nov.). However, within B. timmsi sp. nov. and 
B. scanloni sp. nov., there are phylogenetic clades representing different genetic species that cannot be distinguished morphologically. Of the five clades within $B$. timmsi sp.nov, only 3 are genetically distinct species (clades $\mathrm{A} 1$ and $\mathrm{A} 3$ on the one hand and $\mathrm{A} 2+\mathrm{A} 4+\mathrm{A} 5$ on the other). Within $B$. scanloni sp. nov., the cryptic clades E1/TST and B2 are good genetic species (see below for further remarks on species $B$. timmsi sp. nov. and B. scanloni sp. nov.).

If the topology of the tree in Fig. 2 is correct, then $B$. timmsi sp. nov. may be a polyphyletic species, as clades $\mathrm{A} 1$ and $\mathrm{A} 3$ on the one hand, and clades $\mathrm{A} 2+\mathrm{A} 4+\mathrm{A} 5$ on the other belong to different clusters of the tree. However, the support of the bifurcation between the two groups of $B$. timmsi clades is doubtful (bootstrap value of 77), so the apparent polyphyly of the species may be an artifact. As also no clear morphological characters, distinctive of the clusters, could be found (see below), we decided not to describe these two groups as distinct nominal species. If at some stage in the future this decision is to be reverted, then B. timmsi sp. nov. is defined by clade A5.

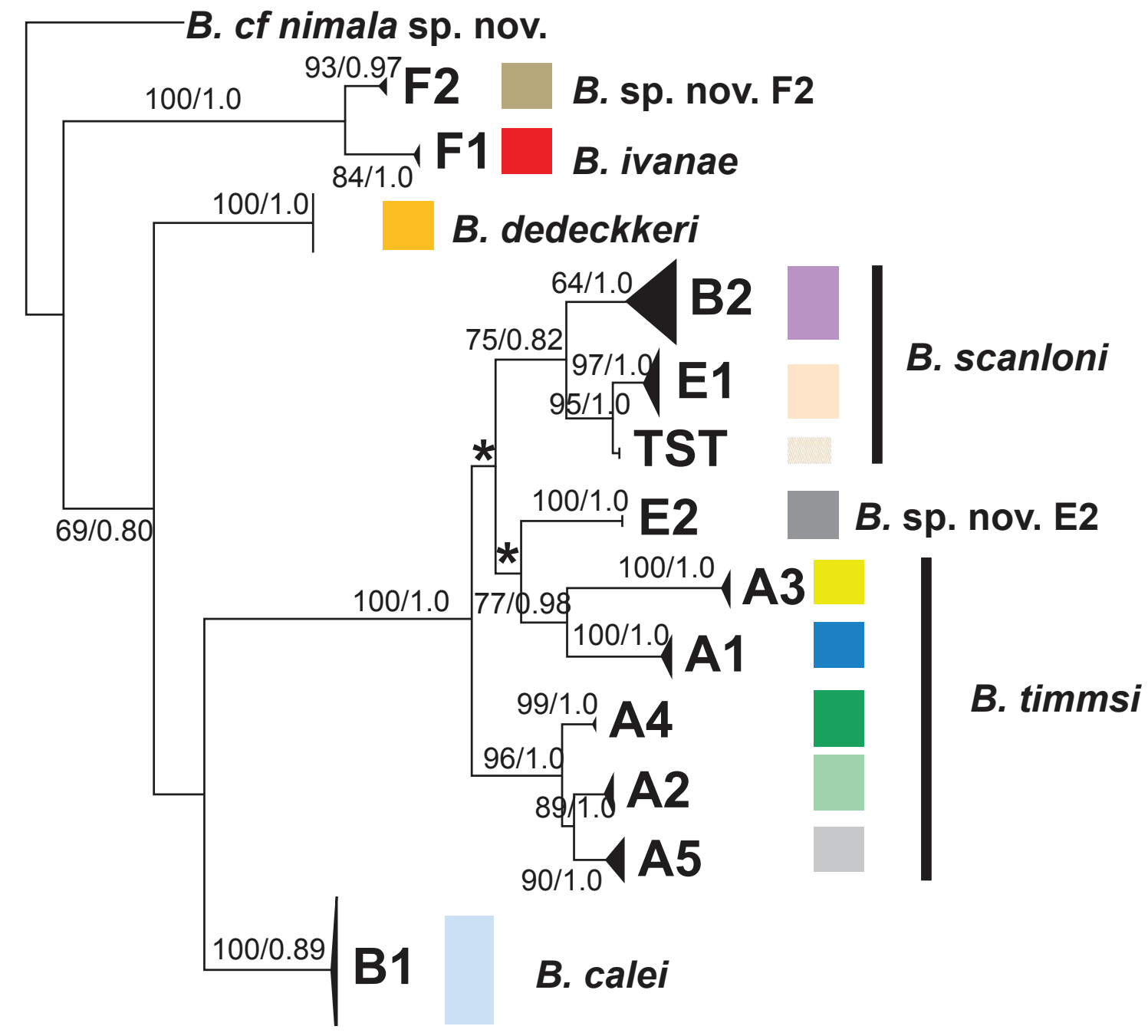

Fig. 2. Phylogenetic tree constructed with Bayesian Inference (BI) and Maximum Likelihood (ML) methods from COI sequences of 99 ostracods from the Bennelongia barangaroo lineage and with $B$. nimala as outgroup. Numbers above nodes illustrate statistical support for this particular node. Numbers before the hash (/) are \% bootstrap values of ML analyses with 1000 replicates, numbers after the hash (/) are Bayesian posterior probabilities (ranging from 0 to 1). Both methods, BI and ML, resulted in the same tree topology. Different phylogenetic clades are indicated by different colours (as in Fig. 3, page 15). The asterisks indicate two nodes ( $\alpha 1$ and $\alpha 2)$ that are weakly supported (see Discussion, p. 55). 
Table 1. Individual measurements of specimens used for the present descriptions. All measurements were done using SEM (see Material and methods). If a molecular sequence was available for the same specimen, the GenBank registration number is also given. However, some specimens were used as whole animals for DNA sequencing, and thus no measurements are available. The present table therefore does not list all 99 specimens for which sequences are available. Specimens in bold are holotypes.

\begin{tabular}{|c|c|c|c|c|c|c|c|c|c|c|c|c|c|}
\hline \multirow[t]{2}{*}{ Mus $\mathrm{Nr}$} & \multirow[t]{2}{*}{ KMWA } & \multirow[t]{2}{*}{$\begin{array}{c}\text { Genbank } \\
\text { accession } \\
\text { number }\end{array}$} & \multirow[t]{2}{*}{$\begin{array}{c}\text { Bennelongia } \\
\text { species }\end{array}$} & \multirow[t]{2}{*}{ locality } & \multirow[t]{2}{*}{$\mathbf{M} / \mathbf{F}$} & \multicolumn{2}{|c|}{ RV } & \multicolumn{2}{|c|}{ LV } & \multicolumn{2}{|c|}{ CpRL } & \multicolumn{2}{|c|}{$\mathrm{CpD} / \mathrm{V}$} \\
\hline & & & & & & $\mathrm{L}$ & $\mathrm{H}$ & $\mathrm{L}$ & $\mathrm{H}$ & $\mathrm{L}$ & $\mathrm{H}$ & $\mathrm{L}$ & $\mathrm{W}$ \\
\hline WAMC52239 & 324 & & timmsi & BVT/010/1 & $\mathrm{F}$ & 1440 & 882 & 1520 & 907 & & & & \\
\hline OC. 3317 & 366 & & timmsi & BVT/10/02 & $\mathrm{F}$ & 1400 & 843 & 1480 & 874 & & & & \\
\hline OC.3317 & 366 & & timmsi & BVT/10/02 & Fext & 1400 & 841 & 1460 & 883 & & & & \\
\hline WAMC52240 & 434 & & timmsi & BVT/10/02 & $\mathrm{M}$ & 1190 & 730 & & & & & & \\
\hline WAMC52240 & 434 & & timmsi & BVT/10/02 & Mext & 1190 & 727 & 1240 & 752 & & & & \\
\hline WAMC52241 & 982 & KF725001 & timmsi & BVT/10/02 & $\mathrm{F}$ & 1420 & 849 & 1490 & 887 & & & & \\
\hline WAMC52242 & 983 & KF725002 & timmsi & BVT/10/02 & $\mathrm{F}$ & 1480 & 903 & 1530 & 931 & & & & \\
\hline WAMC52243 & 984 & KF725003 & timmsi & BVT/10/02 & $\mathrm{F}$ & 1510 & 905 & 1580 & 942 & & & & \\
\hline WAMC52244 & 985 & & timmsi & BVT/10/02 & $\mathrm{M}$ & 1300 & 772 & 1340 & 823 & & & & \\
\hline WAMC52245 & 998 & KF725004 & timmsi & $\mathrm{BVT} / 10 / 03$ & $\mathrm{~F}$ & 1370 & 843 & 1440 & 841 & & & & \\
\hline OC. 3318 & 373 & & timmsi & BVT/10/04 & $\mathrm{F}$ & 1560 & 936 & 1630 & 989 & & & & \\
\hline WAMC52246 & 435 & & timmsi & BVT/10/04 & M & 1340 & 818 & 1410 & 839 & & & & \\
\hline WAMC52246 & 435 & & timmsi & BVT/10/04 & Mext & 1320 & 801 & 1380 & 829 & & & & \\
\hline WAMC52247 & 436 & & timmsi & BVT/10/04 & M & 1380 & 812 & 1440 & 841 & & & & \\
\hline WAMC52248 & 980 & & timmsi & BVT/10/04 & $\mathrm{F}$ & 1540 & 936 & 1650 & 974 & & & & \\
\hline WAMC52249 & 981 & & timmsi & BVT/10/04 & $\mathrm{F}$ & 1580 & 960 & 1660 & 982 & & & & \\
\hline OC.3319 & 986 & & timmsi & BVT/10/04 & M & 1370 & 821 & 1430 & 854 & & & & \\
\hline WAMC52229 & 987 & KF725005 & timmsi & BVT/10/05 & $\mathrm{F}$ & 1510 & 913 & 1600 & 965 & & & & \\
\hline OC.3314 & 988 & KF725006 & timmsi & BVT/10/05 & $\mathrm{F}$ & 1470 & 883 & 1530 & 929 & & & & \\
\hline WAMC52233 & 989 & KF725007 & timmsi & $\mathrm{BVT} / 10 / 05$ & $F$ & 1500 & 891 & 1550 & 925 & & & & \\
\hline WAMC52234 & 990 & & timmsi & BVT/10/05 & F & & & & & & & 1580 & 951 \\
\hline WAMC52235 & 991 & & timmsi & BVT/10/05 & $\mathrm{F}$ & & & & & 1530 & 891 & & \\
\hline OC. 3315 & 992 & & timmsi & BVT/10/05 & $\mathrm{F}$ & & & & & & & 1550 & 931 \\
\hline
\end{tabular}




\begin{tabular}{|c|c|c|c|c|c|c|c|c|c|c|c|c|c|}
\hline \multirow[t]{2}{*}{ Mus $\mathrm{Nr}$} & \multirow[t]{2}{*}{ KMWA } & \multirow[t]{2}{*}{$\begin{array}{c}\text { Genbank } \\
\text { accession } \\
\text { number } \\
\end{array}$} & \multirow[t]{2}{*}{$\begin{array}{l}\text { Bennelongia } \\
\text { species }\end{array}$} & \multirow[t]{2}{*}{ locality } & \multirow[t]{2}{*}{$\mathbf{M} / \mathbf{F}$} & \multicolumn{2}{|c|}{ RV } & \multicolumn{2}{|c|}{ LV } & \multicolumn{2}{|c|}{ CpRL } & \multicolumn{2}{|c|}{$\mathrm{CpD} / \mathrm{V}$} \\
\hline & & & & & & $\mathrm{L}$ & $\mathrm{H}$ & L & $\mathrm{H}$ & $\mathrm{L}$ & $\mathrm{H}$ & L & W \\
\hline OC.3316 & 993 & & timmsi & BVT/10/05 & M & & & & & & & 1370 & 834 \\
\hline WAMC52236 & 994 & & timmsi & BVT/10/05 & M & & & & & 1330 & 770 & & \\
\hline WAMC52237 & 995 & & timmsi & BVT/10/05 & M & & & & & & & 1380 & 840 \\
\hline WAMC52232 & 996 & & timmsi & BVT/10/05 & M & 1283 & 768 & & & & & & \\
\hline WAMC52231 & 997 & & timmsi & BVT/10/05 & M & 1300 & 790 & 1375 & 812 & & & & \\
\hline WAMC52228 & 1073 & & timmsi & BVT/10/05 & M & 1318 & 783 & 1378 & 817 & & & & \\
\hline OC.3312 & 1074 & & timmsi & BVT/10/05 & M & 1320 & 798 & 1375 & 823 & & & & \\
\hline WAMC52230 & 1075 & & timmsi & BVT/10/05 & M & 1285 & 768 & 1337 & 800 & & & & \\
\hline OC. 3313 & 379 & KF725008 & timmsi & BVT/10/05 & $\mathrm{F}$ & 1510 & 901 & 1570 & 950 & & & & \\
\hline OC. 3313 & 379 & & timmsi & BVT/10/05 & Fext & 1490 & 903 & 1550 & 943 & & & & \\
\hline WAMC52250 & 381 & & timmsi & BVT/10/06 & $\mathrm{F}$ & 1500 & 892 & 1560 & 925 & & & & \\
\hline WAMC52250 & 381 & & timmsi & BVT/10/06 & Fext & 1473 & 883 & 1535 & 919 & & & & \\
\hline WAMC52251 & 999 & KF725009 & timmsi & BVT/10/06 & $\mathrm{F}$ & 1510 & 898 & 1535 & 919 & & & & \\
\hline WAMC52252 & 1000 & & timmsi & BVT/10/06 & M & 1329 & 808 & 1404 & 848 & & & & \\
\hline WAMC52253 & 888 & KF725010 & timmsi & BVT/10/08 & M & 1 & 1 & 1404 & 873 & & & & \\
\hline OC. 3320 & 889 & KF725011 & timmsi & BVT/10/08 & $\mathrm{F}$ & 1525 & 940 & 1619 & 981 & & & & \\
\hline WAMC52254 & 890 & KF725012 & timmsi & BVT/10/08 & $\mathrm{F}$ & 1488 & 912 & 1548 & 948 & & & & \\
\hline WAMC52255 & 891 & KF725013 & timmsi & BVT/10/08 & M & 1344 & 814 & 1402 & 829 & & & & \\
\hline WAMC52256 & 892 & & timmsi & BVT/10/08 & M & & & & & & & 1387 & 848 \\
\hline WAMC52257 & 893 & & timmsi & BVT/10/08 & M & & & & & 1390 & 852 & & \\
\hline WAMC52258 & 894 & & timmsi & BVT/10/08 & M & & & & & & & 1390 & 868 \\
\hline WAMC52259 & 895 & & timmsi & BVT/10/08 & $\mathrm{F}$ & & & & & & & 1613 & 987 \\
\hline WAMC52260 & 896 & & timmsi & BVT/10/08 & $\mathrm{F}$ & & & & & & & 1581 & 1015 \\
\hline WAMC52261 & 897 & & timmsi & BVT/10/08 & $\mathrm{F}$ & & & & & 1588 & 939 & & \\
\hline WAMC52262 & 901 & & timmsi & BVT/10/08 & M & 1335 & 835 & 1396 & 840 & & & & \\
\hline OC. 3321 & 902 & & timmsi & BVT/10/08 & M & 1327 & 821 & 1398 & 842 & & & & \\
\hline OC. 3322 & 222 & & gnamma & OSTR012A & $\mathrm{F}$ & 1450 & 973 & 1520 & 1050 & & & & \\
\hline
\end{tabular}




\begin{tabular}{|c|c|c|c|c|c|c|c|c|c|c|c|c|c|}
\hline \multirow[t]{2}{*}{ Mus Nr } & \multirow[t]{2}{*}{ KMWA } & \multirow{2}{*}{$\begin{array}{c}\text { Genbank } \\
\text { accession } \\
\text { number }\end{array}$} & \multirow[t]{2}{*}{$\begin{array}{c}\text { Bennelongia } \\
\text { species }\end{array}$} & \multirow[t]{2}{*}{ locality } & \multirow[t]{2}{*}{$\mathbf{M} / \mathbf{F}$} & \multicolumn{2}{|c|}{$\mathbf{R V}$} & \multicolumn{2}{|c|}{$\mathbf{L V}$} & \multicolumn{2}{|c|}{ CpRL } & \multicolumn{2}{|c|}{$\mathrm{CpD} / \mathrm{V}$} \\
\hline & & & & & & $\mathrm{L}$ & $\mathrm{H}$ & $\mathrm{L}$ & $\mathrm{H}$ & $\mathrm{L}$ & $\mathrm{H}$ & $\mathrm{L}$ & $\mathrm{W}$ \\
\hline WAMC52266 & 223 & & gnamma & OSTR012A & $\mathrm{F}$ & & & & & & & 1560 & 1000 \\
\hline WAMC52263 & OS178 & & gnamma & OSTR012A & $\mathbf{F}$ & 1550 & 915 & 1620 & 991 & & & & \\
\hline WAMC52277 & 226 & & hirsuta & OSTR012D & $\mathrm{F}$ & & & & & 1430 & 826 & & \\
\hline WAMC52272 & 227 & & hirsuta & OSTR012D & $\mathrm{M}$ & & & & & 1450 & 837 & 1447 & 855 \\
\hline WAMC52270 & 1101 & & hirsuta & OSTR012D & F & 1400 & 842 & 1470 & 845 & & & & \\
\hline WAMC52274 & 1104 & & hirsuta & OSTR012D & $\mathrm{F}$ & 1390 & 813 & 1450 & 824 & & & & \\
\hline OC.3324 & 1102 & & hirsuta & OSTR012D & $\mathrm{F}$ & & & & & 1410 & 803 & & \\
\hline WAMC52279 & 903 & & hirsuta & $\mathrm{BVT} / 10 / 09$ & $\mathrm{~F}$ & 1217 & 719 & 1302 & 719 & & & & \\
\hline WAMC52280 & 851 & KF725014 & ivanae & DJC/02 & $\mathbf{F}$ & 1475 & 915 & 1533 & 948 & & & & \\
\hline WAMC52280 & 851 & & ivanae & DJC/02 & Fext & 1452 & 904 & 1512 & 940 & & & & \\
\hline OC.3326 & 852 & KF725015 & ivanae & DJC/02 & $\mathrm{F}$ & 1460 & 910 & 1542 & 947 & & & & \\
\hline OC.3326 & 852 & & ivanae & $\mathrm{DJC} / 02$ & Fext & 1456 & 904 & 1513 & 935 & & & & \\
\hline OC. 3327 & 1001 & & ivanae & $\mathrm{DJC} / 02$ & $\mathrm{~F}$ & & & & & 1488 & 931 & & \\
\hline WAMC52281 & 1002 & & ivanae & $\mathrm{DJC} / 02$ & $F$ & & & & & & & 1498 & 1025 \\
\hline WAMC52282 & 1003 & & ivanae & $\mathrm{DJC} / 02$ & $\mathrm{~F}$ & & & & & & & 1452 & / \\
\hline
\end{tabular}




\begin{tabular}{|c|c|c|c|c|c|c|c|c|c|c|c|c|c|}
\hline \multirow[t]{2}{*}{ Mus Nr } & \multirow[t]{2}{*}{ KMWA } & \multirow[t]{2}{*}{$\begin{array}{c}\text { Genbank } \\
\text { accession } \\
\text { number }\end{array}$} & \multirow[t]{2}{*}{$\begin{array}{c}\text { Bennelongia } \\
\text { species }\end{array}$} & \multirow[t]{2}{*}{ locality } & \multirow[t]{2}{*}{$\mathbf{M} / \mathbf{F}$} & \multicolumn{2}{|c|}{$\mathbf{R V}$} & \multicolumn{2}{|c|}{ LV } & \multicolumn{2}{|c|}{ CpRL } & \multicolumn{2}{|c|}{$\mathrm{CpD} / \mathrm{V}$} \\
\hline & & & & & & L & $\mathrm{H}$ & L & $\mathrm{H}$ & L & $\mathrm{H}$ & $\mathrm{L}$ & W \\
\hline WAMC52289 & 1078 & & mcraeae & OSTR014B & F & & & & & 1650 & 1000 & & \\
\hline WAMC52324 & 295 & & scanloni & BRYDE7 & F & 1460 & 873 & 1560 & 895 & & & & \\
\hline WAMC52324 & 295 & & scanloni & BRYDE7 & Fext & 1448 & 863 & 1550 & 992 & & & & \\
\hline WAMC52325 & 297 & & scanloni & BRYDE7 & F & 1460 & 863 & 1550 & 887 & & & & \\
\hline WAMC52325 & 297 & & scanloni & BRYDE7 & Fext & 1438 & 860 & 1533 & 883 & & & & \\
\hline WAMC52326 & 299 & & scanloni & BRYDE7 & $\mathrm{F}$ & & & & & 1489 & 878 & 1455 & 858 \\
\hline OC.3339 & 194 & & scanloni & BRYDE7 & $\mathrm{F}$ & 1330 & 807 & 1044 & 839 & & & & \\
\hline OC.3339 & 194 & & scanloni & BRYDE7 & Fext & 1322 & 803 & 1412 & 822 & & & & \\
\hline OC.3340 & 437 & & scanloni & OSTR013C & $\mathrm{F}$ & 1310 & 775 & 1380 & 788 & & & & \\
\hline OC.3340 & 437 & & scanloni & OSTR013C & Fext & 1303 & 765 & 1350 & 782 & & & & \\
\hline WAMC52327 & 438 & & scanloni & OSTR013C & $\mathrm{M}$ & 1260 & 751 & 1310 & 761 & & & & \\
\hline WAMC52327 & 438 & & scanloni & OSTR013C & Mext & 1242 & 738 & 1303 & 749 & & & & \\
\hline WAMC52328 & 1107 & & scanloni & OSTR013C & $\mathrm{M}$ & 1230 & 688 & 1320 & 709 & & & & \\
\hline \multirow[t]{2}{*}{ OC.3338 } & 916 & & scanloni & TST & M & 1248 & 756 & 1331 & 769 & & & & \\
\hline & 917 & & scanloni & TST & $\mathrm{F}$ & 1394 & 810 & 1487 & 833 & & & & \\
\hline WAMC52322 & 918 & KF724982 & scanloni & TST & $\mathrm{F}$ & 1294 & 752 & 1396 & 711 & & & & \\
\hline WAMC52323 & 919 & & scanloni & TST & F & & & & & & & 1352 & 744 \\
\hline OC.3334 & 855 & & scanloni & DJC/09 & $\mathrm{F}$ & 1438 & 900 & 1485 & 930 & & & & \\
\hline OC.3334 & 855 & & scanloni & DJC/09 & Fext & 1446 & 890 & 1490 & 898 & & & & \\
\hline WAMC52310 & 856 & & scanloni & DJC/09 & $\mathrm{F}$ & 1465 & 923 & 1525 & 933 & & & & \\
\hline WAMC52310 & 856 & & scanloni & DJC/09 & Fext & 1421 & 898 & 1477 & 910 & & & & \\
\hline WAMC52304 & 831 & KF724983 & scanloni & DJC/11 & F & 1380 & 797 & 1463 & 807 & & & & \\
\hline WAMC52304 & 831 & & scanloni & DJC/11 & Fext & 1360 & 773 & 1433 & 796 & & & & \\
\hline \multirow[t]{2}{*}{ lost } & 832 & KF724984 & scanloni & $\mathrm{DJC} / 11$ & $\mathrm{~F}$ & 1367 & 803 & 1458 & 807 & & & & \\
\hline & 832 & & scanloni & DJC/11 & Fext & 1352 & 789 & 1438 & 808 & & & & \\
\hline WAMC52297 & 837 & & scanloni & $\mathrm{DJC} / 11$ & $\mathrm{~F}$ & & & & & & & 1497 & 910 \\
\hline WAMC52298 & 838 & & scanloni & $\mathrm{DJC} / 11$ & $\mathrm{~F}$ & & & & & & & 1448 & 832 \\
\hline
\end{tabular}




\begin{tabular}{|c|c|c|c|c|c|c|c|c|c|c|c|c|c|}
\hline \multirow[t]{2}{*}{ Mus Nr } & \multirow[t]{2}{*}{ KMWA } & \multirow[t]{2}{*}{$\begin{array}{c}\text { Genbank } \\
\text { accession } \\
\text { number }\end{array}$} & \multirow[t]{2}{*}{$\begin{array}{c}\text { Bennelongia } \\
\text { species }\end{array}$} & \multirow[t]{2}{*}{ locality } & \multirow[t]{2}{*}{$\mathbf{M} / \mathbf{F}$} & \multicolumn{2}{|c|}{ RV } & \multicolumn{2}{|c|}{$\mathbf{L V}$} & \multicolumn{2}{|c|}{ CpRL } & \multicolumn{2}{|c|}{$\mathrm{CpD} / \mathrm{V}$} \\
\hline & & & & & & $\mathrm{L}$ & $\mathrm{H}$ & $\mathrm{L}$ & $\mathrm{H}$ & L & $\mathrm{H}$ & $\mathrm{L}$ & $\mathrm{W}$ \\
\hline WAMC52295 & 841 & & scanloni & $\mathrm{DJC} / 11$ & M & & & & & 1290 & 730 & 1302 & 731 \\
\hline WAMC52296 & 842 & & scanloni & $\mathrm{DJC} / 11$ & M & & & & & & & 1222 & 745 \\
\hline WAMC52293 & 1006 & & scanloni & $\mathrm{DJC} / 11$ & M & 1244 & 723 & 1327 & 735 & & & & \\
\hline WAMC52294 & 1007 & & scanloni & $\mathrm{DJC} / 11$ & $\mathrm{M}$ & 1190 & 696 & 1263 & 708 & & & & \\
\hline WAMC52292 & 1008 & KF724985 & scanloni & $\mathrm{DJC} / 11$ & $\mathrm{~F}$ & 1263 & 752 & 1356 & 775 & & & & \\
\hline OC.3331 & 1009 & & scanloni & $\mathrm{DJC} / 11$ & $\mathrm{~F}$ & 1312 & 777 & 1398 & 792 & & & & \\
\hline WAMC52303 & 1013 & KF724987 & scanloni & $\mathrm{DJC} / 11$ & $\mathrm{~F}$ & 1485 & 883 & 1588 & 906 & & & & \\
\hline OC. 3335 & 1022 & KF724988 & scanloni & $\mathrm{DJC} / 19$ & $\mathrm{~F}$ & 1446 & 885 & 1519 & 906 & & & & \\
\hline WAMC52311 & 1023 & KF724989 & scanloni & $\mathrm{DJC} / 19$ & $\mathrm{~F}$ & 1 & 1 & 1660 & broken & & & & \\
\hline WAMC52312 & 1025 & & scanloni & $\mathrm{DJC} / 19$ & $\mathrm{~F}$ & & & & & 1515 & 887 & & \\
\hline WAMC52313 & 1026 & & scanloni & $\mathrm{DJC} / 19$ & $\mathrm{~F}$ & & & & & & & 1475 & 1 \\
\hline WAMC52314 & 1029 & & scanloni & $\mathrm{DJC} / 19$ & $\mathrm{~F}$ & & & & & & & 1713 & 1038 \\
\hline WAMC52315 & 1030 & & scanloni & $\mathrm{DJC} / 19$ & $\mathrm{~F}$ & & & & & & & 1721 & 1035 \\
\hline OC.3336 & 1031 & & scanloni & $\mathrm{DJC} / 19$ & $\mathrm{~F}$ & & & & & 1625 & 983 & & \\
\hline OC.3337 & 797 & & scanloni & $\mathrm{DJC} / 23$ & $\mathrm{~F}$ & 1573 & 973 & 1670 & 1015 & & & & \\
\hline OC.3337 & 797 & & scanloni & $\mathrm{DJC} / 23$ & Fext & 1560 & 979 & 1633 & 987 & & & & \\
\hline
\end{tabular}




\begin{tabular}{|c|c|c|c|c|c|c|c|c|c|c|c|c|c|}
\hline \multirow[t]{2}{*}{ Mus Nr } & \multirow[t]{2}{*}{ KMWA } & \multirow[t]{2}{*}{$\begin{array}{c}\text { Genbank } \\
\text { accession } \\
\text { number }\end{array}$} & \multirow[t]{2}{*}{$\begin{array}{l}\text { Bennelongia } \\
\text { species }\end{array}$} & \multirow[t]{2}{*}{ locality } & \multirow[t]{2}{*}{$\mathbf{M} / \mathbf{F}$} & \multicolumn{2}{|c|}{ RV } & \multicolumn{2}{|c|}{ LV } & \multicolumn{2}{|c|}{ CpRL } & \multicolumn{2}{|c|}{$\mathrm{CpD} / \mathrm{V}$} \\
\hline & & & & & & $\mathrm{L}$ & $\mathrm{H}$ & $\mathrm{L}$ & $\mathrm{H}$ & $\mathrm{L}$ & $\mathrm{H}$ & $\mathrm{L}$ & W \\
\hline WAMC52307 & 909 & KF724992 & scanloni & BVT/10/10 & F & 1415 & 885 & 1465 & 898 & & & & \\
\hline WAMC52308 & 910 & KF724993 & scanloni & $\mathrm{BVT} / 10 / 10$ & F & 1602 & 994 & 1656 & 1015 & & & & \\
\hline OC.3333 & 911 & & scanloni & BVT/10/10 & M & 1277 & 1 & 1350 & 831 & & & & \\
\hline WAMC52336 & 822 & KF724994 & calei & DJC/18 & $\mathrm{F}$ & 1340 & 802 & 1432 & 818 & & & & \\
\hline WAMC52336 & 822 & & calei & $\mathrm{DJC} / 18$ & Fext & broken & 792 & 1419 & 814 & & & & \\
\hline WAMC52335 & 823 & KF724995 & calei & DJC/18 & F & 1480 & 857 & 1555 & 865 & & & & \\
\hline WAMC52335 & 823 & & calei & DJC/18 & Fext & 1452 & 844 & 1552 & 862 & & & & \\
\hline OC.3344 & 1014 & & calei & $\mathrm{DJC} / 18$ & F & 1446 & 835 & 1531 & 848 & & & & \\
\hline WAMC52339 & 1015 & & calei & $\mathrm{DJC} / 18$ & $\mathrm{~F}$ & 1490 & 864 & 1588 & 873 & & & & \\
\hline WAMC52340 & 1016 & & calei & $\mathrm{DJC} / 18$ & F & 1335 & 775 & 1408 & 864 & & & & \\
\hline WAMC52341 & 1017 & KF724996 & calei & $\mathrm{DJC} / 18$ & F & 1244 & 729 & 1337 & 748 & & & & \\
\hline OC.3345 & 828 & & calei & DJC/18 & $\mathrm{F}$ & & & & & 1477 & 815 & & \\
\hline WAMC52337 & 829 & & calei & $\mathrm{DJC} / 18$ & F & & & & & & & 1477 & 827 \\
\hline WAMC52338 & 830 & & calei & $\mathrm{DJC} / 18$ & $\mathrm{~F}$ & & & & & & & 1518 & 802 \\
\hline WAMC52355 & 236 & & calei & SPM017B & F & 1150 & 686 & 1240 & 711 & & & & \\
\hline WAMC52355 & 236 & & calei & SPM017B & Fext & 1145 & 682 & 1232 & 703 & & & & \\
\hline WAMC52356 & 237 & & calei & SPM017B & $\mathrm{F}$ & & & & & 1290 & 735 & 1290 & 729 \\
\hline & 238 & & calei & SPM017B & $\mathrm{F}$ & & & & & & & 1180 & 695 \\
\hline WAMC52353 & 195 & & calei & SPM017B & $\mathrm{F}$ & & & & & 1330 & 756 & 1325 & 718 \\
\hline WAMC52354 & 196 & & calei & SPM017B & F & 1170 & 699 & 1260 & 720 & & & & \\
\hline WAMC52354 & 196 & & calei & SPM017B & Fext & 1167 & 692 & 1252 & 713 & & & & \\
\hline WAMC52349 & 870 & KF724997 & calei & $\mathrm{DJC} / 10$ & $\mathrm{~F}$ & 1422 & 833 & 1522 & 855 & & & & \\
\hline WAMC52349 & 870 & & calei & $\mathrm{DJC} / 10$ & Fext & 1400 & 837 & 1508 & 848 & & & & \\
\hline OC.3346 & 807 & & calei & BVT/11/04 & F & 1307 & 792 & 1382 & 797 & & & & \\
\hline OC.3347 & 808 & & calei & BVT/11/04 & F & & & & & & & 1427 & 782 \\
\hline WAMC52343 & 809 & & calei & BVT/11/04 & $\mathrm{F}$ & & & & & 1397 & 783 & & \\
\hline WAMC52344 & 810 & & calei & BVT/11/04 & $\mathrm{F}$ & & & & & & & 1425 & 772 \\
\hline
\end{tabular}




\begin{tabular}{|c|c|c|c|c|c|c|c|c|c|c|c|c|c|}
\hline \multirow[t]{2}{*}{ Mus Nr } & \multirow[t]{2}{*}{ KMWA } & \multirow[t]{2}{*}{$\begin{array}{c}\text { Genbank } \\
\text { accession } \\
\text { number }\end{array}$} & \multirow[t]{2}{*}{$\begin{array}{l}\text { Bennelongia } \\
\text { species }\end{array}$} & \multirow[t]{2}{*}{ locality } & \multirow[t]{2}{*}{$\mathbf{M} / \mathbf{F}$} & \multicolumn{2}{|c|}{ RV } & \multicolumn{2}{|c|}{ LV } & \multicolumn{2}{|c|}{ CpRL } & \multicolumn{2}{|c|}{ CpD/V } \\
\hline & & & & & & $\mathrm{L}$ & $\mathrm{H}$ & $\mathrm{L}$ & $\mathrm{H}$ & $\mathrm{L}$ & $\mathrm{H}$ & $\mathrm{L}$ & W \\
\hline WAMC52345 & 813 & KF724998 & calei & BVT/11/05 & $\mathrm{F}$ & 1383 & 812 & 1460 & 832 & & & & \\
\hline WAMC52345 & 813 & & calei & BVT/11/05 & Fext & 1371 & 812 & 1449 & 832 & & & & \\
\hline WAMC52346 & 814 & & calei & BVT/11/05 & F & & & & & & & 1473 & 828 \\
\hline WAMC52347 & 815 & & calei & BVT/11/05 & F & & & & & & & 1417 & 773 \\
\hline WAMC52348 & 816 & & calei & BVT/11/05 & $\mathrm{F}$ & & & & & 1443 & 808 & & \\
\hline WAMC52329 & 1080 & KF724999 & calei & $\mathrm{DJC} / 15$ & $\mathrm{~F}$ & 1 & 1 & 1587 & 906 & & & & \\
\hline OC.3343 & 1081 & & calei & DJC/15 & $\mathrm{F}$ & & & & & 1540 & 889 & & \\
\hline WAMC52330 & 1082 & & calei & $\mathrm{DJC} / 15$ & $\mathrm{~F}$ & & & & & & & 1546 & 842 \\
\hline WAMC52331 & 1083 & & calei & DJC/15 & $\mathrm{F}$ & & & & & & & 1506 & 842 \\
\hline OC.3348 & 874 & KF725000 & calei & $\mathrm{DJC} / 36$ & $\mathrm{~F}$ & 1345 & 817 & 1433 & 833 & & & & \\
\hline OC.3349 & 879 & & calei & DJC/36 & $\mathrm{F}$ & & & & & & & 1513 & 843 \\
\hline WAMC52350 & 880 & & calei & $\mathrm{DJC} / 36$ & F & & & & & 1497 & 850 & & \\
\hline WAMC52351 & 881 & & calei & DJC/36 & F & & & & & & & 1430 & 830 \\
\hline WAMC52352 & 882 & & calei & $\mathrm{DJC} / 36$ & F & & & & & 1410 & 780 & & \\
\hline OC.3350 & 190 & & dedeckkeri & KIES10 & F & & & & & 1110 & 650 & & \\
\hline WAMC52359 & 191 & & dedeckkeri & KIES10 & $\mathrm{F}$ & & & & & & & 1100 & 641 \\
\hline WAMC52360 & 192 & & dedeckkeri & KIES10 & $\mathrm{F}$ & & & & & & & 1080 & 651 \\
\hline WAMC52357 & 193 & & dedeckkeri & KIES10 & F & 1110 & 676 & 1190 & 701 & & & & \\
\hline OC.3351 & 678 & & dedeckkeri & SIKE9 & $\mathrm{F}$ & 1125 & 672 & 1188 & 715 & & & & \\
\hline OC. 3352 & 679 & & dedeckkeri & SIKE9 & F & & & & & 1210 & 717 & & \\
\hline WAMC52364 & 680 & & dedeckkeri & SIKE9 & F & & & & & & & 1187 & 652 \\
\hline WAMC52365 & 681 & & dedeckkeri & SIKE9 & $\mathrm{F}$ & & & & & & & 1145 & 660 \\
\hline
\end{tabular}


Table 2. Results of tests for genetic species boundaries using the 4 theta method for six species of the Bennelongia barangaroo lineage.

\begin{tabular}{|c|c|c|c|c|c|}
\hline $\begin{array}{l}\text { Phylogenetic sister } \\
\text { clades }\end{array}$ & Species & $\begin{array}{l}\text { max. } \theta \\
\text { (within clades) }\end{array}$ & $\begin{array}{l}\text { D (between } \\
\text { clades) }\end{array}$ & Ratio D/O & $\mathbf{n}^{1}, \mathbf{n}^{2}$ \\
\hline \multirow[t]{2}{*}{ A1-A3 } & \multirow{2}{*}{ B. timmsi } & 0.0076 & 0.114 & 15.00 & \multirow{2}{*}{8,7} \\
\hline & & 0.0078 & 0.139 & 17.82 & \\
\hline \multirow[t]{2}{*}{ A2-A4 } & \multirow{2}{*}{ B. timmsi } & 0.0138 & 0.052 & 3.77 & \multirow{2}{*}{9,3} \\
\hline & & 0.0141 & 0.058 & 4.11 & \\
\hline \multirow[t]{2}{*}{ A2-A5 } & \multirow{2}{*}{ B. timmsi } & 0.0138 & 0.048 & 3.48 & \multirow{2}{*}{8,8} \\
\hline & & 0.0141 & 0.052 & 3.69 & \\
\hline \multirow[t]{2}{*}{ A4-A5 } & \multirow{2}{*}{ B. timmsi } & 0.0138 & 0.049 & 3.55 & \multirow{2}{*}{3,5} \\
\hline & & 0.0141 & 0.054 & 3.83 & \\
\hline \multirow[t]{2}{*}{ A1-E1 } & B. timmsi- & 0.0133 & 0.131 & 9.85 & \multirow{2}{*}{8,13} \\
\hline & B. scanloni & 0.0138 & 0.169 & 12.25 & \\
\hline \multirow[t]{2}{*}{ A3-E1 } & B. timmsi- & 0.0133 & 0.140 & 10.53 & \multirow{2}{*}{7,13} \\
\hline & B. scanloni & 0.0138 & 0.182 & 13.19 & \\
\hline \multirow[t]{2}{*}{ B1-B. dedeckkeri } & B. calei - & 0.004 & 0.126 & 31.5 & 23 \\
\hline & B. dedeckkeri & 0.004 & 0.161 & 40.25 & 25 \\
\hline \multirow[t]{2}{*}{ B2-E1 } & \multirow{2}{*}{ B. scanloni } & 0.020 & 0.113 & 5.65 & 15 \\
\hline & & 0.021 & 0.141 & 6.71 & 13 \\
\hline \multirow[t]{2}{*}{ B2-DJC11 } & \multirow{2}{*}{ B. scanloni } & 0.050 & 0.040 & 0.80 & \multirow{2}{*}{15,2} \\
\hline & & 0.053 & 0.044 & 0.83 & \\
\hline \multirow[t]{2}{*}{ E1-TST } & \multirow{2}{*}{ B. scanloni } & 0.0133 & 0.032 & 2.41 & \multirow{2}{*}{13,2} \\
\hline & & 0.0138 & 0.034 & 2.46 & \\
\hline \multirow[t]{2}{*}{ B2-E2 } & B. scanloni- & 0.020 & 0.103 & 5.15 & \multirow{2}{*}{15,2} \\
\hline & B. sp. nov. E2 & 0.020 & 0.127 & 6.35 & \\
\hline \multirow{2}{*}{ E2-TST } & B. scanloni- & 0.0015 & 0.109 & 72.67 & \multirow{2}{*}{2,2} \\
\hline & B. sp. nov. E2 & 0.0015 & 0.135 & 90.00 & \\
\hline \multirow[t]{2}{*}{ F1-F2 } & B. ivanae- & 0.0058 & 0.061 & 10.52 & \multirow{2}{*}{3,4} \\
\hline & B. sp. nov. F2 & 0.0059 & 0.067 & 11.36 & \\
\hline
\end{tabular}

$\theta=$ population genetic parameter theta, indicating genetic variability within populations. $\mathrm{D}=$ genetic distance between sister clades. $\mathrm{n}^{1}, \mathrm{n}^{2}=$ number of sequences for each sister clade. $\theta$ and $\mathrm{D}$ were either calculated as $\mathrm{p}$ distance or with the Tamura-3 parameter model (in italics). Nearest neighbours or sister clades were defined from the COI tree constructed with Bayesian Inference and Maximum Likelihood methods (see Fig. 2). In order to fulfill the criteria of the 4 theta rule for species status, the ratio of the mean sequence diversity within as compared to between the two nearest neighbours of one sister clade needs to be 4 or more, depending on the number of specimens per clade (Birky et al. 2010). Comparisons, for which these criteria are fulfilled, are printed in bold. 


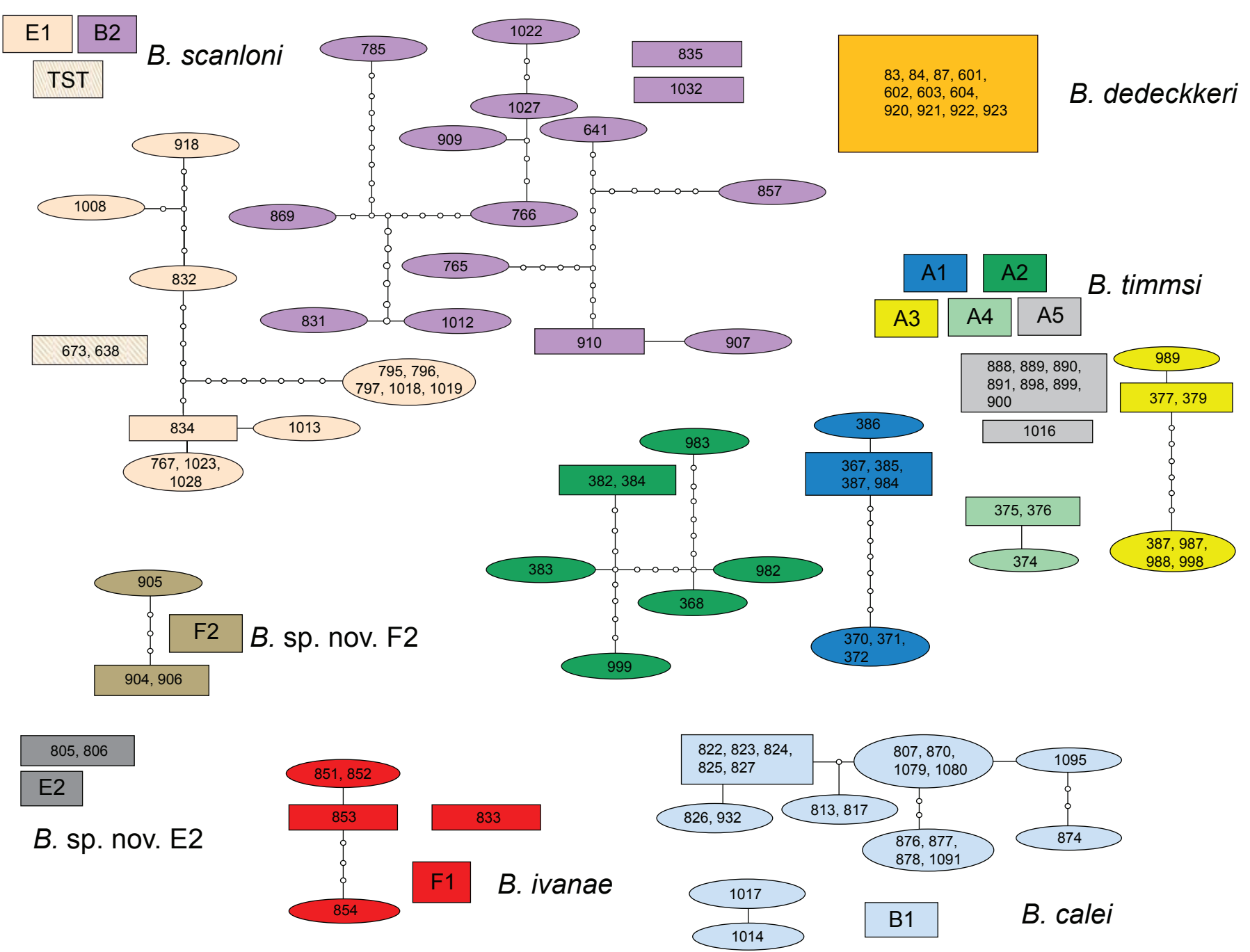

Fig. 3. Parsimonius network, based on COI sequences of the Bennelongia barangaroo lineage. Squares represent ancestral sequences (or haplotypes), small circles missing haplotypes. The size of squares and large ovals is proportional to the number of individuals with the same sequence in the analysed population. The network was constructed at the $95 \%$ probability limit, which includes up to 8 mutation steps for connecting different sequences or haplotypes. Different phylogenetic clades are indicated by different colours, which match those used in Fig. 2 (page 6). 
The structure of the most parsimonious networks in Fig. 3 also reflects the higher genetic than morphological diversity. However, there are two species with a single haplotype each, $B$. dedeckkeri and $B$. spec. E2 sp. nov. (clade E2). For the latter, we obtained COI sequences of two individuals only while the same (identical) haplotype is found in 11 specimens of $B$. dedeckkeri. The three individuals in clade F2 (and B. spec. F2 sp. nov.) share two haplotypes, while the five specimens of B. ivanae sp. nov. possess 4 haplotypes, of which one remains unconnected. The remaining three species display two (B. calei sp. nov.) to five (B. scanloni sp. nov.) and six (B. timmsi sp. nov.) unconnected haplotype networks, respectively. The different genetic species within $B$. timmsi sp. nov. and $B$. scanloni sp. nov. form unconnected haplotype networks.

\section{Taxonomic descriptions}

Class Ostracoda Latreille, 1806

Subclass Podocopa G.O. Sars, 1866

Order Podocopida G.O. Sars, 1866

Suborder Cypridocopina Baird, 1845

Superfamily Cypridoidea Baird, 1845

Family Cyprididae Baird, 1845

Subfamily Bennelongiinae Martens et al., 2012

Genus Bennelongia De Deckker \& McKenzie, 1981

\section{Diagnosis}

See Martens et al. (2012)

\section{Bennelongia barangaroo lineage}

\section{Remarks}

De Deckker (1981a) described B. barangaroo from Lake Buchanan (QLD - Type locality), but also reported the same species from other localities in QLD, NSW, SA, WA and New Zealand. However, as in De Deckker's (1981a) re-description of B. australis (Brady, 1886) (see Martens et al. 2012), at least two different species within this lineage were illustrated under the same name. The (type) specimens of B. barangaroo in De Deckker's (1981a) figure 7 from Lake Buchanan have a short, sub-quadrate, slightly ventrally pointed lapel on the antero-ventral side of the RV. The specimens from a pool $25 \mathrm{~km}$ $\mathrm{N}$ of Cue (WA) (De Deckker 1981a: fig. 9), however, appear to have an elongated lapel, much as is the case in B. calei sp. nov. (see below). We thus decided previously (Shearn et al. 2012) that the true $B$. barangaroo needs to be established based on new material from the type locality. Fortunately, De Deckker (1981a) illustrated the valves and soft parts of the holotype male, which facilitated identification, and allowed Shearn et al. (2012) to confidently describe B. dedeckkeri as a different species within the B. barangaroo lineage. Shearn et al. (2012) also described B. mckenziei as a second new species from QLD, characterised by a total absence of the lapel on the RV.

\section{Diagnosis of the B. barangaroo lineage}

All species of the $B$. barangaroo lineage (re-)described here share a number of features: all have relatively elongated and wide (in dorsal view) carapaces, mostly green in colour, relatively smooth (but hirsute) in adults. The RV has an internal eyelet at the posteroventral internal side, mostly situated directly internally of the lapel. This eyelet is best visible with transparent light; although in most species it is also visible on SEM micrographs (see various illustrations below). 


\author{
Bennelongia timmsi sp. nov. \\ Figs 4-11 \\ urn:Isid:zoobank.org:act:4F6A6E8F-5636-4290-85A4-B234D5DA4466
}

\title{
Diagnosis
}

Valves in inner view (Fig. 4A-B, D-E) relatively high, with greatest height situated well in front of the middle; ventral margin anteriorly with well-pronounced mandibular curve. LV (Fig. 4A, D) with anterior il not overlapping. RV (Fig. 4B, E) with antero-ventral lapel subtriangular, asymmetrically produced with a ventral point (Fig. 4K-M). Carapace in dorsal and ventral views (Fig. 4G-J) with greatest width in the middle, hirsute, anteriorly with a mild rostrum; in lateral views (Fig. 4C, F) showing a clear anterior $\mathrm{LV}>\mathrm{RV}$ overlap.

Hemipenes (holotype: Fig. 8F) mostly symmetrical, with ls protruding well beyond ventral tip of ms, ls with broad base, ventrally bluntly beak-shaped. Right prehensile palp (holotype: Fig. 8D) with distal segment elongated, with dorsal margin evenly rounded. Left prehensile palp (holotype: Fig. 8E) with distal segment elongated, reaching beyond ventro-apical margin of proximal segment with at least half of its length.

\section{Etymology}

This species is named after Prof. Brian V. Timms (Newcastle, Australia), in recognition of his vast contribution to the knowledge of Australian non-marine crustaceans in general, and of phyllopods from temporary pools in particular. Prof. Timms also collected the material of the present species from a series of pools on various rocky outcrops in WA.

Measurements (all measurements in $\mu \mathrm{m}$ - see Table 1 for measurements of all specimens illustrated with SEM)

Holotype $\widehat{\jmath}$ (WAMC52228): RV: $\mathrm{L}=1318, \mathrm{H}=783$. LV: $\mathrm{L}=1378, \mathrm{H}=817$.

Allotype $q$ (WAMC52229): RV: $\mathrm{L}=1510, \mathrm{H}=913 . \mathrm{LV}: \mathrm{L}=1600, \mathrm{H}=965$.

\section{Type locality}

Rock pools on Wave Rock, WA, ca. $2 \mathrm{~km}$ E of Hyden. Approximate coordinates: $32^{\circ} 27^{\prime} \mathrm{S} 118^{\circ} 54^{\prime} \mathrm{E}$ (WGS 84). Material handpicked from pools by B.V. Timms on 23 Jul. 2010 (sample BVT/10/05).

\section{Type material}

\section{Holotype}

$\widehat{\partial}$ (WAMC52228), with soft parts dissected in a sealed slide and valves stored dry in a micropalaeontological slide.

\section{Allotype}

q (WAMC52229), with soft parts dissected in a sealed slide, and valves stored dry in a micropalaeontological slide.

\section{Paratypes}

Numerous males and females from the type locality, either dissected and stored as the holotype, or as

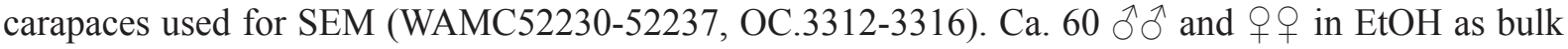
paratypes (WAMC52238).

\section{Other material investigated}

All material from WA, collected by B.V. Timms. 


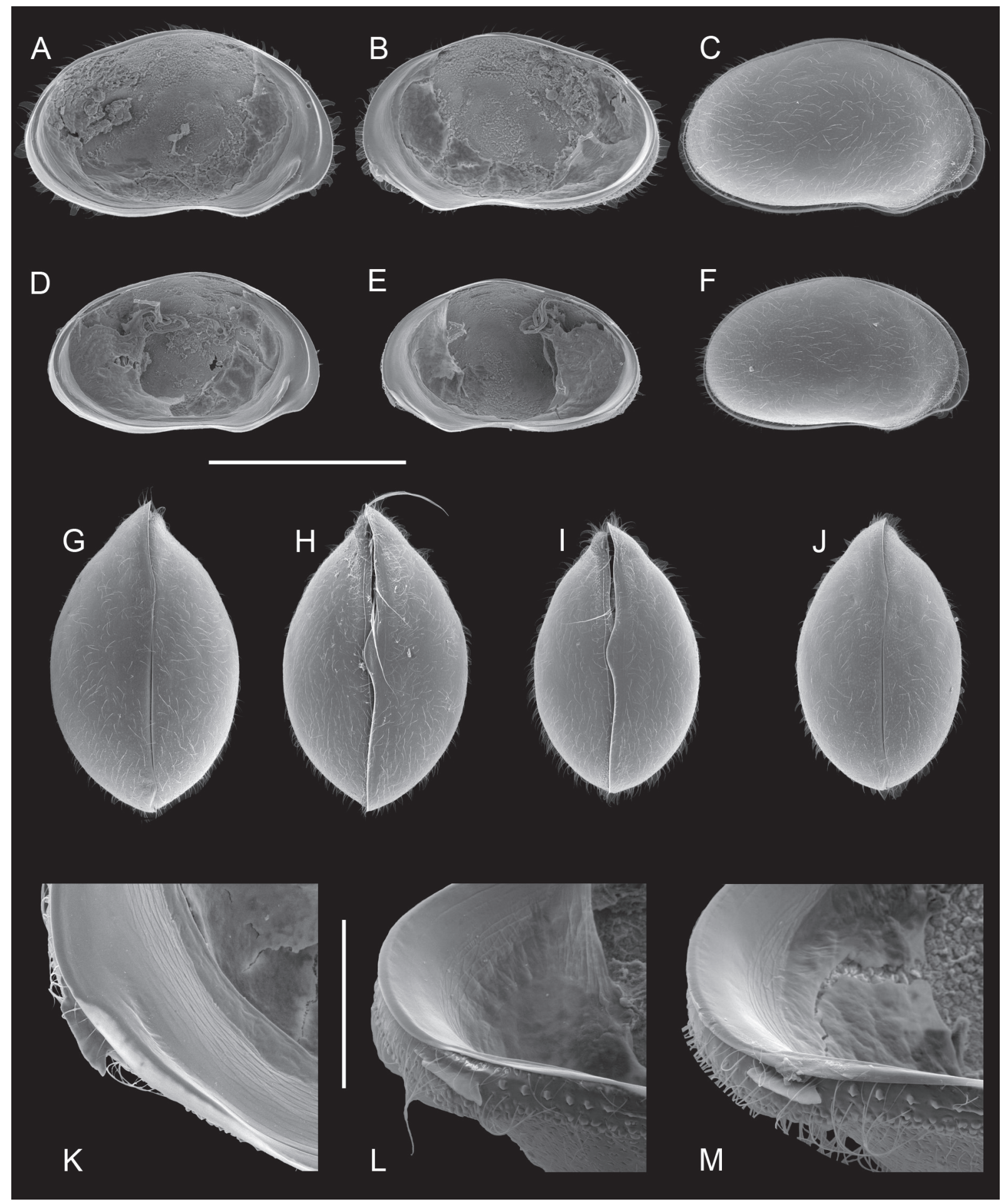

Fig. 4. Bennelongia timmsi sp. nov., all represent paratypes from Wave Rock (BVT/10/05 - type locality). A. + , LVi (OC.3313). B. + , RVi (idem). C. क , CpRL (WAMC52235). D. § holotype, LVi

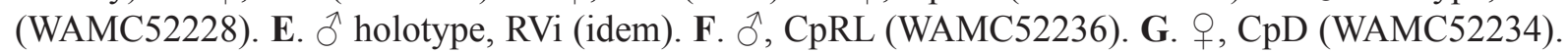
H. + , $\mathrm{CpV}$ (OC.3315). I. O, $\mathrm{CpV}$ (OC.3316). J. O, CpD (WAMC52237). K. O holotype, RVi, detail anterior (WAMC52228). L. ô holotype, RVi, detail anterior, tilted (WAMC52228). M. , , LVi, detail anterior, tilted (OC.3313). Scales: A-J $=1 \mathrm{~mm} ; \mathrm{K}-\mathrm{M}=200 \mu \mathrm{m}$. 


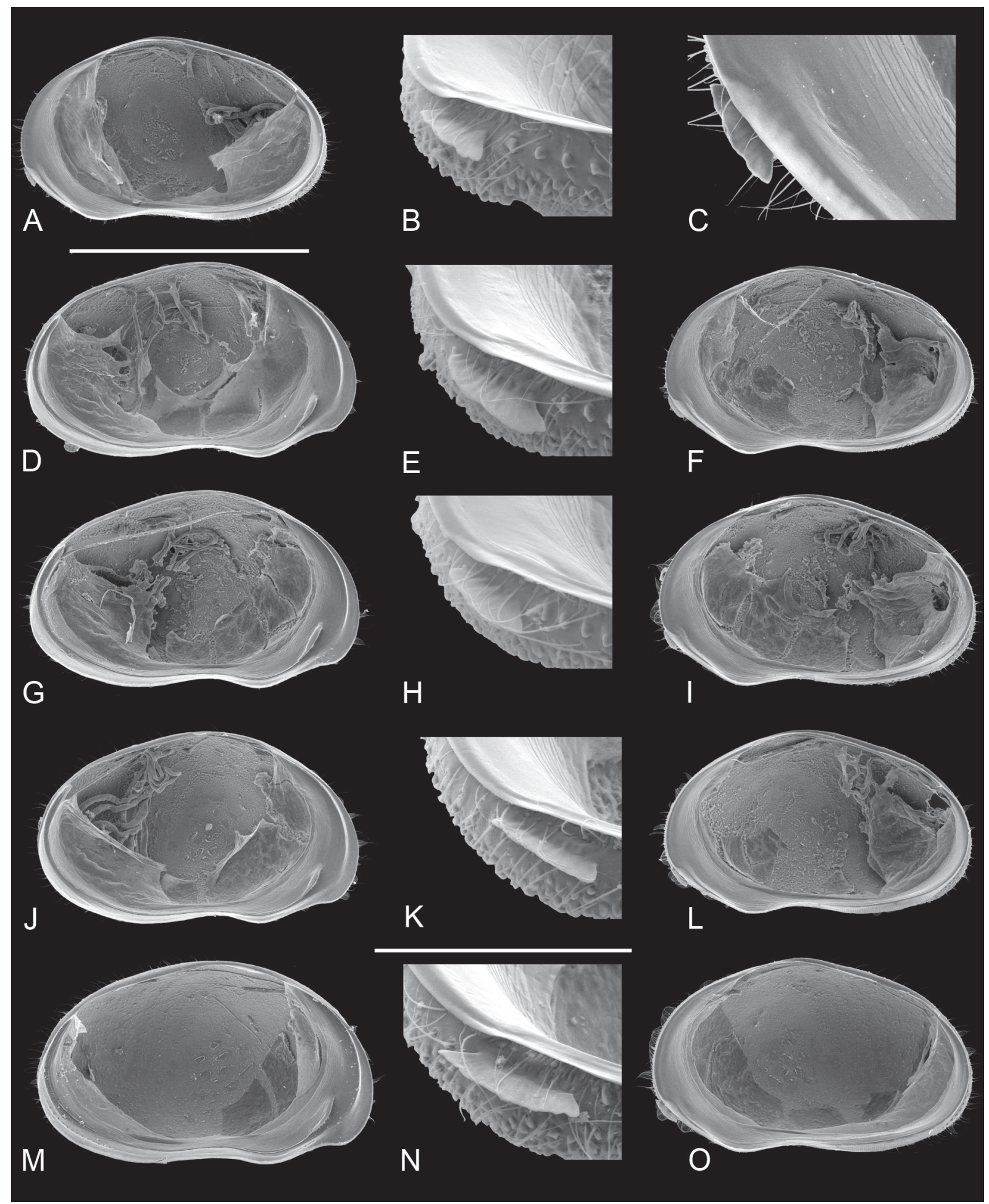

Fig. 5. Bennelongia timmsi sp. nov., all males with hemipenes and prehensiles palps illustrated (see Figs 8-10). A-L = paratypes from Wave Rock $(\mathrm{BVT} / 10 / 05), \mathrm{M}-\mathrm{O}=$ non-types from King Rocks (BVT/10/06). - A-C. WAMC52232: A. RVi. B. RVi, detail anterior, tilted. C. RVi, detail anterior. D-F. WAMC52231: D. LVi. E. RVi, detail anterior, tilted. F. RVi. - G-I. OC.3312: G. LVi. H. RVi, detail anterior, tilted. I. RVi. - J-L. WAMC52230: J. LVi. K. RVi, detail anterior, tilted. L. RVi. M-O. WAMC52252: M. LVi. N. RVi, detail anterior, tilted. O. RVi. Scales: A, D, F-G, I-J, L-M, O = 1 $\mathrm{mm}$; B-C, E, H, K, N = $200 \mu \mathrm{m}$. 


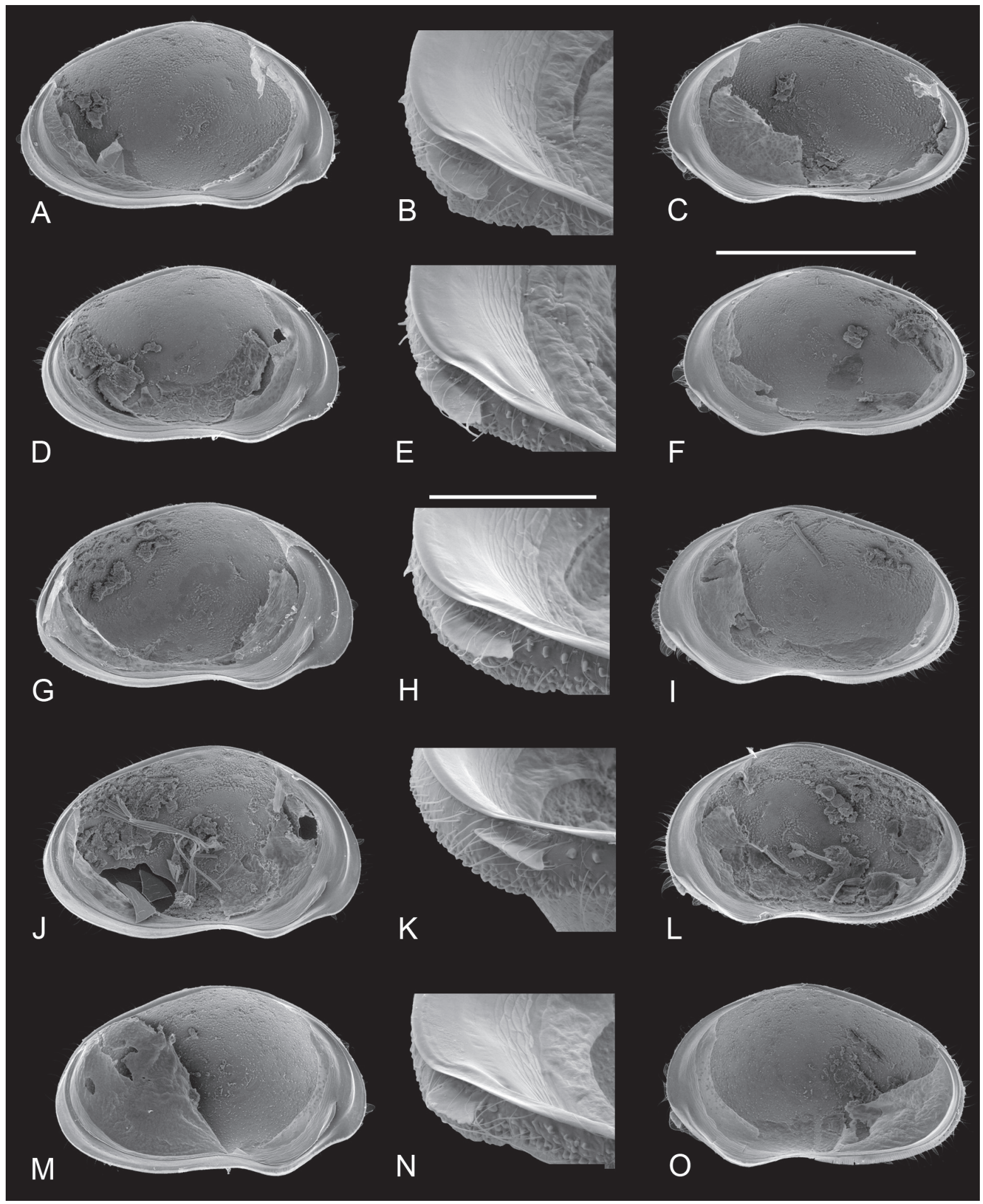

Fig. 6. Bennelongia timmsi sp. nov., all represent females with molecular data available. - A-C. Grahams Rock (BVT/10/02 - WAMC52243 - cryptic species A1): A. LVi. B. RVi, detail anterior, tilted. C. RVi. - D-F. Grahams Rock (BVT/10/02 - WAMC52241 - cryptic species A2): D. LVi. E. RVi, detail anterior, tilted. F. RVi. - G-I. King Rocks (BVT/10/06 - WAMC52251 - cryptic species A2): G. LVi. H. RVi, detail anterior, tilted. I. RVi. - J-L. Wave Rock (BVT/10/05 - allotype WAMC52229 - cryptic species A3): J. LVi. K. RVi, detail anterior, tilted. L. RVi. - M-O. Mt Madden Rocks (BVT/10/08 WAMC52254 - cryptic species A5): M. LVi. N. RVi, detail anterior, tilted. O. RVi. Scales: A, C-D, F-G, I-J, L-M, O = $1 \mathrm{~mm}$; B, E, H, K, N = $200 \mu \mathrm{m}$. 


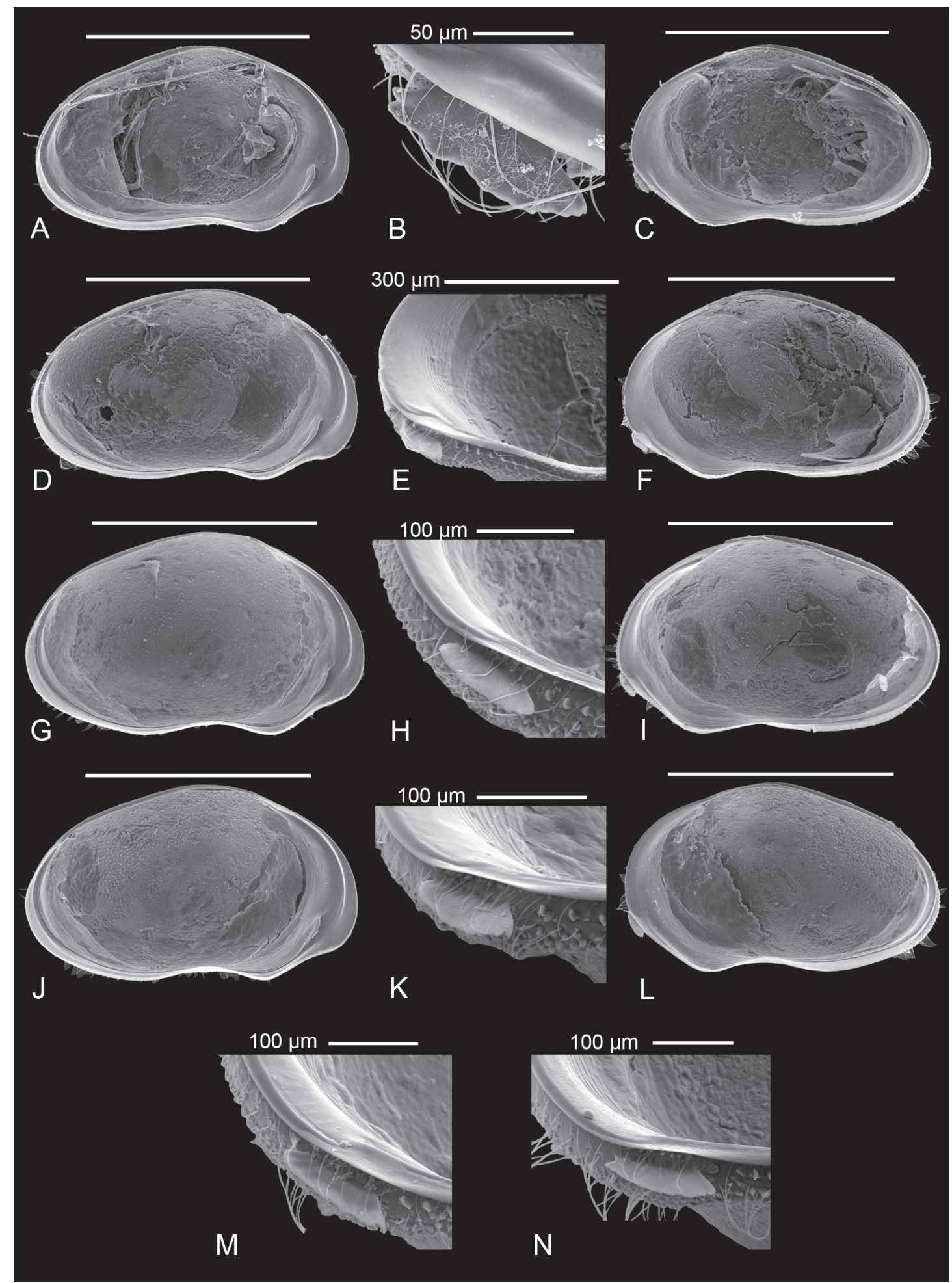

Fig. 7. Bennelongia timmsi sp. nov. - A-C. Mt Madden Rocks (BVT/10/08 - §, WAMC52255 cryptic species A5). A. LVi. B. RVi, detail anterior, tilted. C. RVi. - D-F. Anderson Rock (BVT/10/03 +, WAMC52245). D. LVi. E. RVi, detail anterior, tilted. F. RVi. — G-I. Paynes Find Rock (BVT/10/01 - +, WAMC52239). G. LVi. H. RVi, detail anterior, tilted. I. RVi. - J-L. Grahams Rock (BVT/10/02 - , OC.3317). J. LVi. K. RVi, detail anterior, tilted. L. RVi. - M. Burracopin Rock (BVT/10/04 - 9 , OC.3318), RVi, detail anterior, tilted. - N. King Rocks (BVT/10/06 - +, WAMC52250), RVi, detail anterior, tilted. Scales $=1 \mathrm{~mm}$ unless otherwise indicated. 


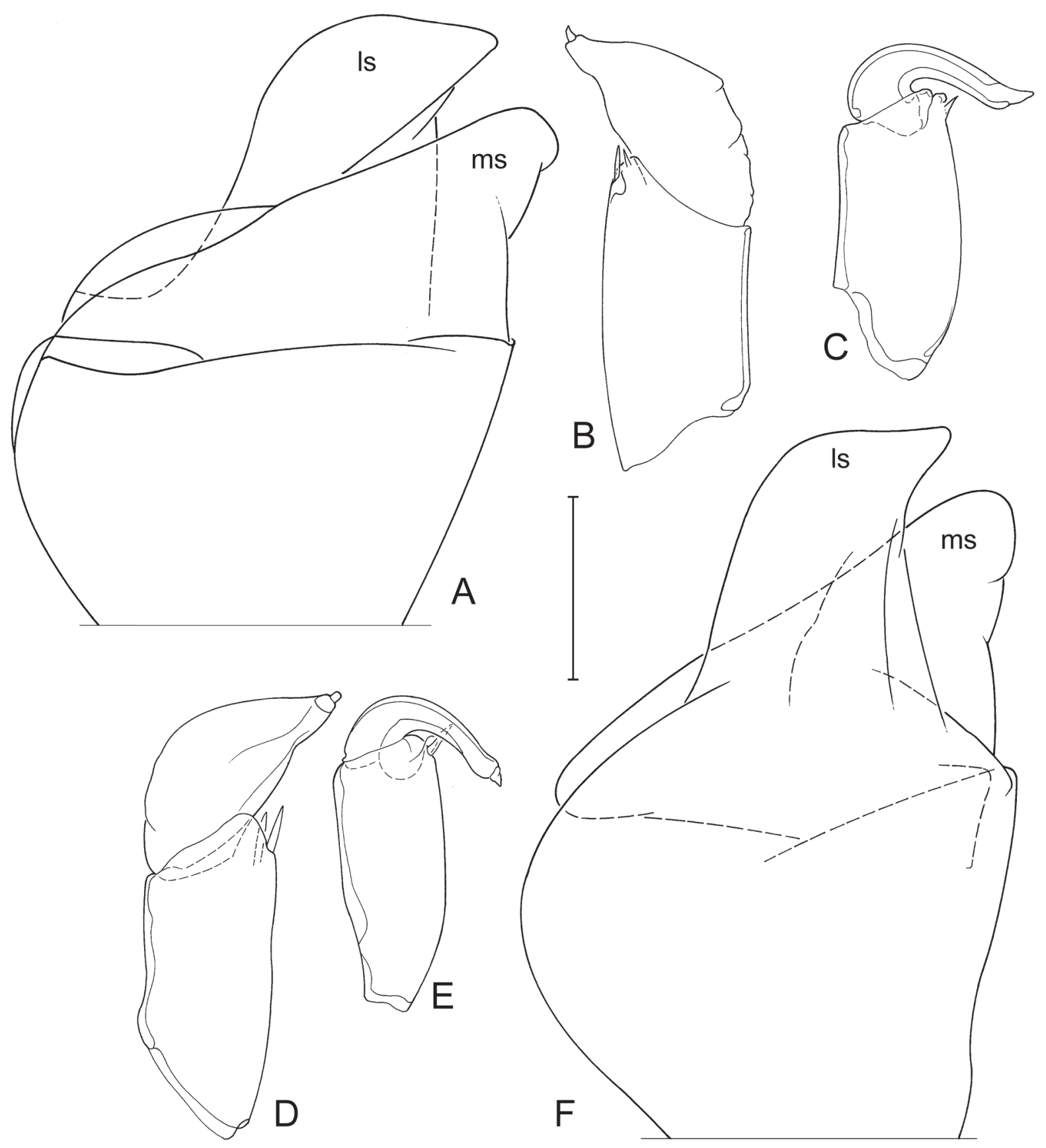

Fig. 8. Bennelongia timmsi sp. nov., male type specimens from type locality (Wave Rock, BVT/10/05). - A-C. Paratype M (OC.3312): A. Hemipenis (both hemipenes symmetrical in this specimen). B. Right prehensile palp. C. Left prehensile palp. - D-F. Holotype $\sigma^{\lambda}$ (WAMC52228): D. Right prehensile palp. E. Left prehensile palp. F. Hemipenis (both hemipenes symmetrical in this specimen). Scale: A-F $=92 \mu \mathrm{m}$. 

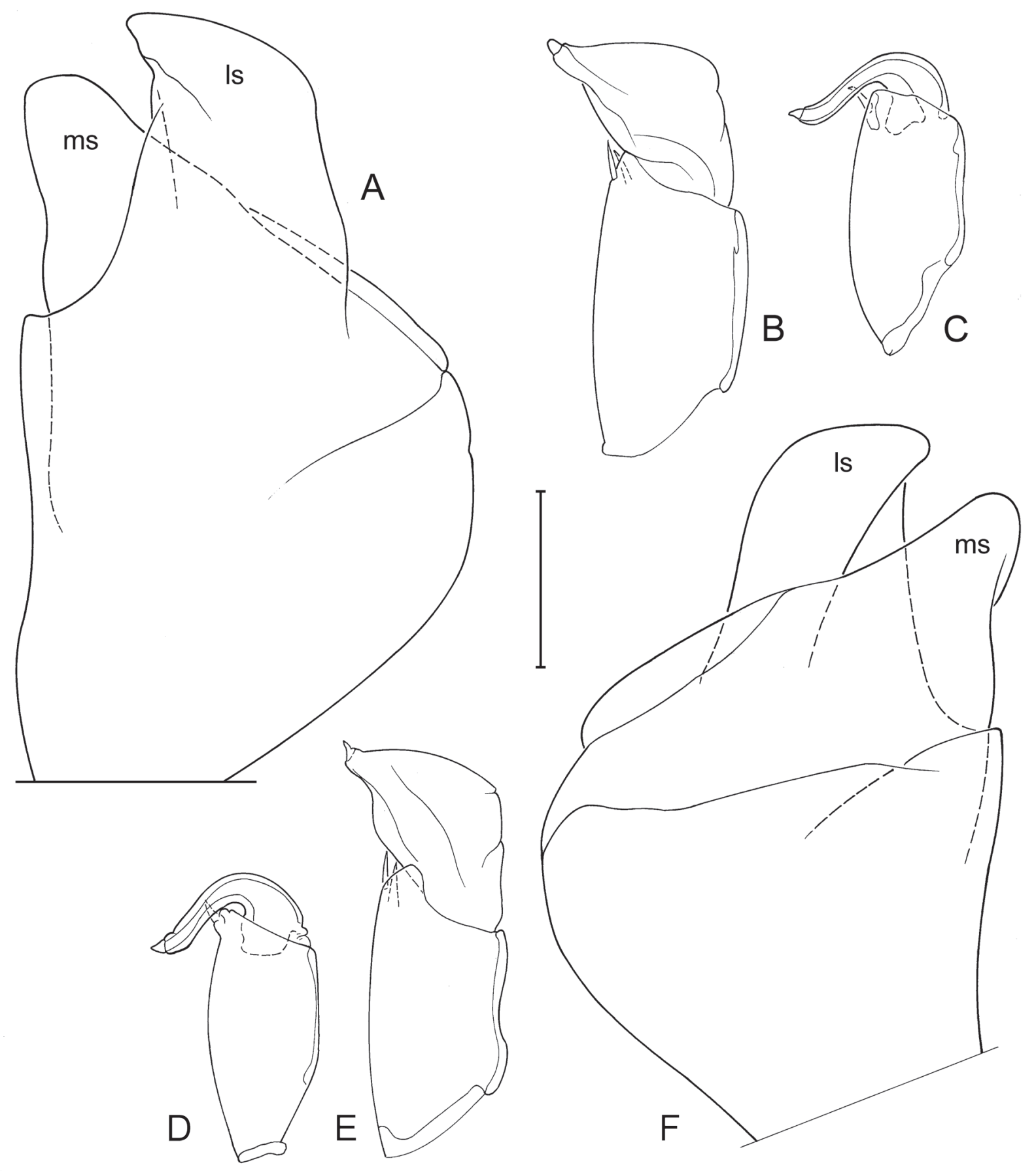

Fig. 9. Bennelongia timmsi sp. nov., paratypes from type locality (Wave Rock, BVT/10/05). - A-C. Paratype (WAMC52231): A. Hemipenis (both hemipenes symmetrical in this specimen). B. Right prehensile palp. C. Left prehensile palp. - D-F. Paratype $\widehat{\curlywedge}$ (WAMC52230): D. Left prehensile palp. E. Right prehensile palp. F. Hemipenis (both hemipenes symmetrical in this specimen). Scale: A-F=92 $\mu \mathrm{m}$. 


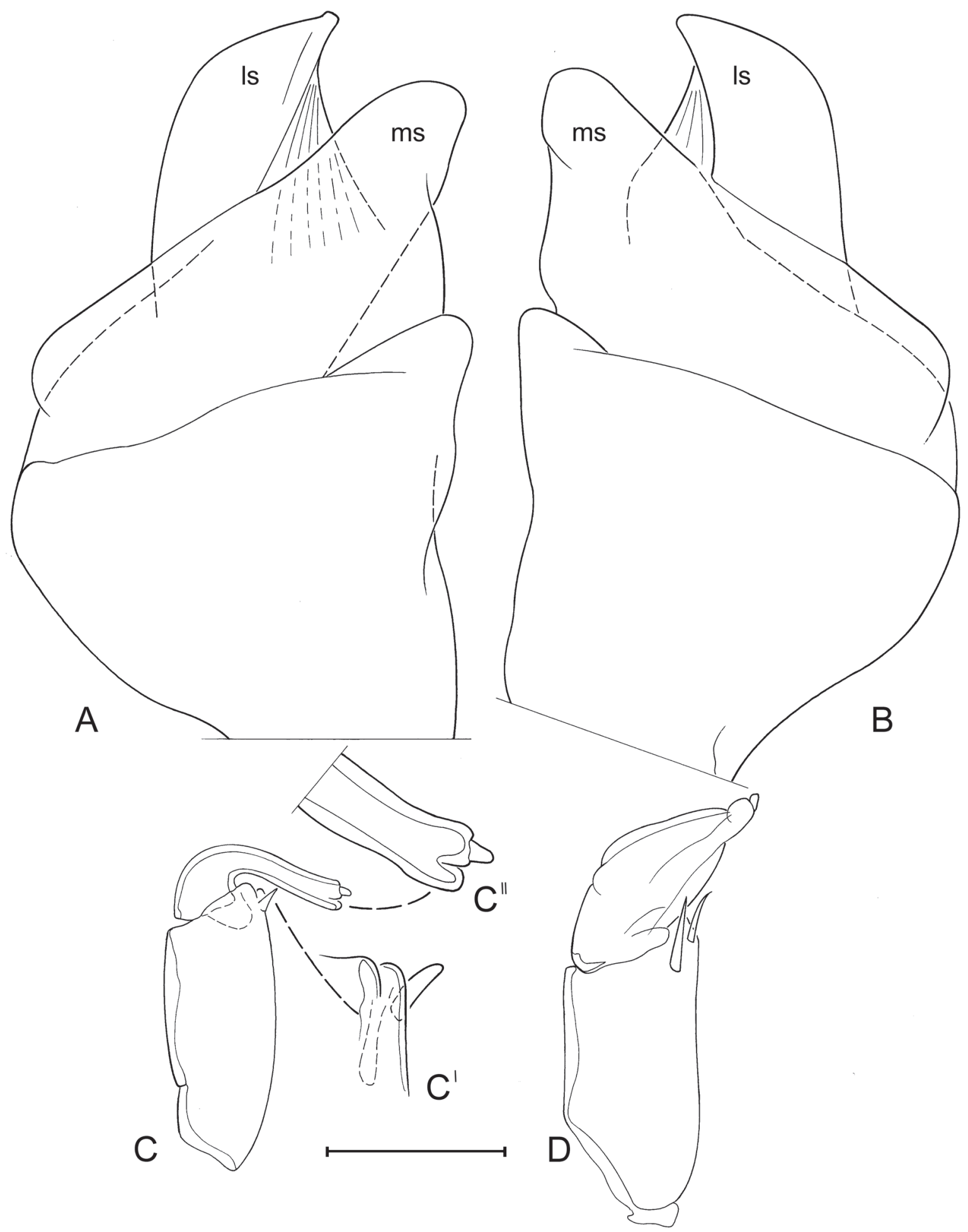

Fig. 10. Bennelongia timmsi sp. nov., male paratype (WAMC52232) from type locality (Wave Rock, BVT/10/05) Aberrant specimen. A. Hemipenis. B. Hemipenis. C. Left prehensile palp. C'. Idem, detail of ventroapical part of first segment, showing two lobes and a sensory organ. C". Idem, detail of distal part of second segment, showing aberrant, bilobed morphology. D. Right prehensile palp. Scale: A-D = $92 \mu \mathrm{m} ; \mathrm{C}^{\prime}, \mathrm{C}^{\prime \prime}=37 \mu \mathrm{m}$. 


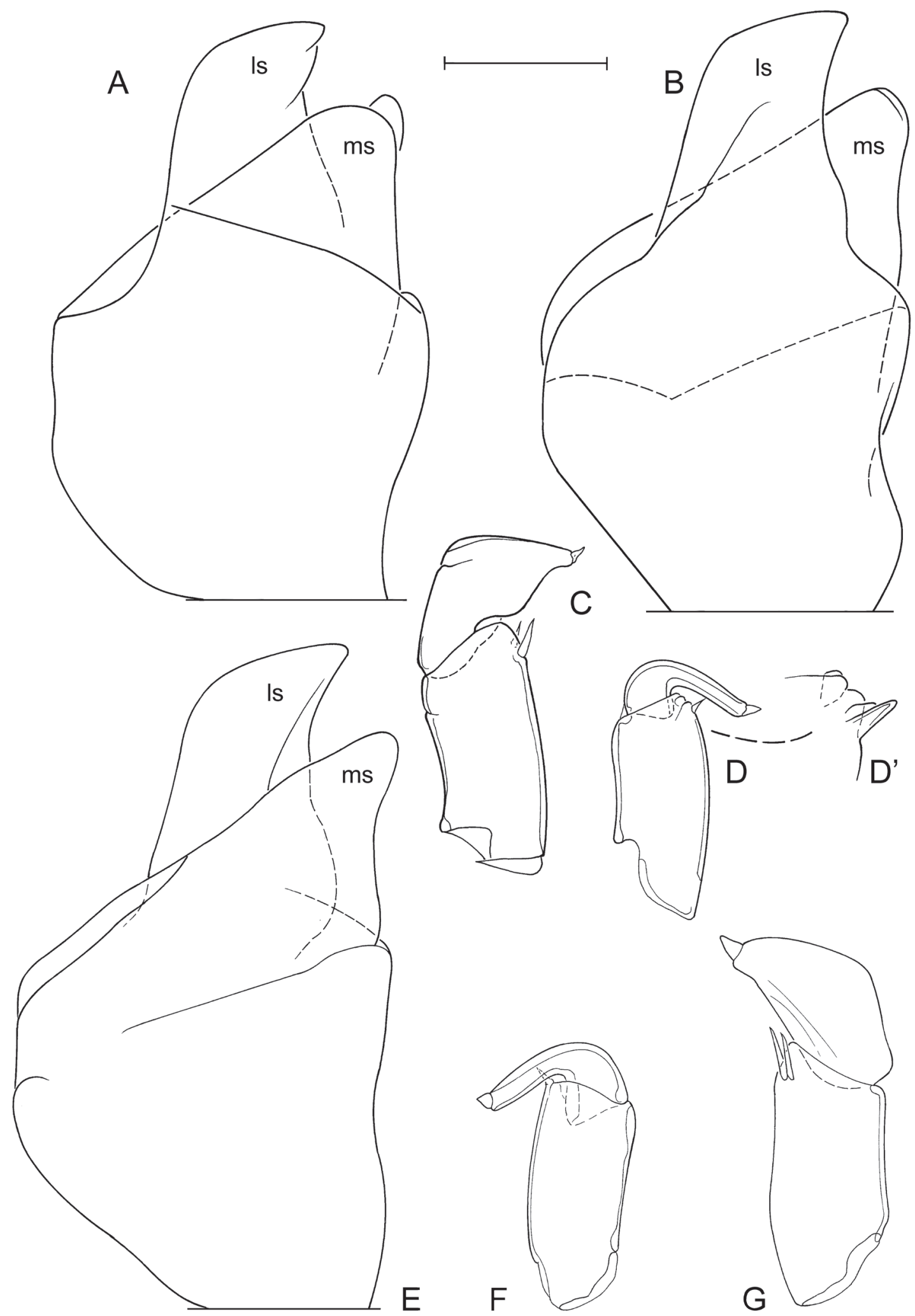

Fig. 11. Bennelongia timmsi sp. nov., non-type males. - A-D. Grahams Rock (WAMC52240, BVT/10/02): A. Hemipenis. B. Hemipenis. C. Right prehensile palp. D. Left prehensile palp. D'. Idem, detail of ventro-apical part of first segment. - E-F. King Rocks (WAMC52252, BVT/10/06): E. Hemipenis. F. Left prehensile palp. G. Right prehensile palp. Scale: A-G $=92 \mu \mathrm{m}$; D' $=37 \mu \mathrm{m}$. 


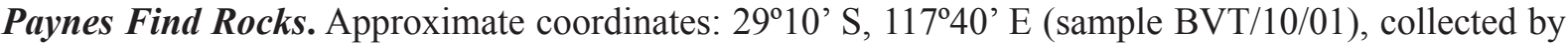
B.V. Timms on 23 Jul. 2010 (one $q$ - WAMC52239).

Grahams Rock. 32²8' S, 11903' E (sample BVT/10/02), collected by B.V. Timms on 23 Jul. 2010 (six ${ }^{\circ}{ }^{\lambda}$ and 웅, WAMC52240-52244; OC.3317).

Anderson Rock. 32 $12^{\circ}$ ' S, 118 51' E (sample BVT/10/03), collected by B.V. Timms on 23 Jul. 2010 (one o, WAMC52245).

Burracopin Rock. $31^{\circ} 24^{\prime}$ S, $118^{\circ} 27^{\prime}$ E (sample BVT/10/04), collected by B.V. Timms on 26 Jul. 2010 (six $\widehat{\partial} \widehat{\delta}$ and + + + , WAMC52246-52249; OC.3318-3319).

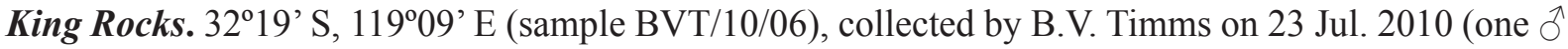
and two 0 우, WAMC52250-52252).

Yorkrakine Rocks. $31^{\circ} 25^{\prime}$ S, 117 30' E (sample BVT/10/07), collected by B.V. Timms on 27 Jul. 2010. Mt Madden Rock. 33 ${ }^{\circ} 14$ ' 22" S, 119 50' 33” E (sample BVT/10/08), collected by B.V. Timms on 01 Aug. 2010 (11 $\widehat{\delta}$ and $q$ + , WAMC52253-52262; OC.332-3321).

\section{Differential diagnosis}

Bennelongia timmsi sp. nov. can be distinguished from most species of the $B$. barangaroo lineage by the triangular and ventrally pointed lapel on the RV and the strongly sinuous ventral valve margins. The lapel of $B$. scanloni sp. nov. is also subtriangular and ventrally protruding, but it is rounded, thus looking drop-shaped in internal (non-tilted) view.

Bennelongia timmsi sp. nov. can moreover be distinguished from B. gnamma sp. nov. by the less high and less rounded valves and by the ls of the hemipenes, which protrudes well beyond the ms (subequal in B. gnamma sp. nov.).

\section{Additional notes on cryptic species}

As was described above, five genetic clusters are recognised in this species (A1-5, Fig. 2). According to the calculations of the 4 theta rule, three cryptic species were found in B. timmsi sp. nov. with molecular methods, but no morphological diagnostic features could be found. Cryptic species A1 occurred in BVT/10/02, 03 and 07. Cryptic species A3 was found in BVT/10/02, 04, 06 and 08. Cryptic species $\mathrm{A} 2+\mathrm{A} 4+\mathrm{A} 5$ occurred in BVT/10/03 and 05 and is used here to characterize B. timmsi sp. nov. with BVT/10/05 (Wave Rock) as type locality. Note that BVT/10/02 (Grahams Rock) and BVT/10/03 (Anderson Rock) hold at least two sympatric clades/cryptic species each. In order to establish beyond reasonable doubt that the specimens belonging to these clusters and cryptic species are indeed morphologically indistinguishable, long series of specimens are illustrated.

Sample BVT/10/05 from pools on Wave Rock appeared to contain only one genetic cluster and cryptic species and, for this reason, Wave Rock was chosen as type locality. We then proceeded with two different approaches: (1) to dissect a series of males from this sample to test whether male reproductive organs (hemipenes, prehensile palps) showed uniformity within one cluster/cryptic species; (2) we checked for potential differences in the morphology of the valves of specimens belonging to different populations and/or shown to belong to different clusters/cryptic species.

\section{Type specimens}

Valves and carapaces of males and females of the type population (in sample BVT/10/05) were illustrated (Fig. 4) and this morphology defines the species. We then dissected several males from the same sample and population and illustrated the soft part and valve morphology. Shape of valves and size and shape of the antero-ventral lapel on the RV were most similar and indeed almost indistinguishable (Fig. 5). In all male specimens the valves have the shape described in the diagnosis above. The lapels are all elongated subtriangular, with a more or less serrated distal margin. In tilted perspective some lapels appear to be 
shorter than others (e.g., the lapel in Fig. 5B appears shorter than in 5E), but this is almost entirely a matter of distorted perspective depending on how the valves were positioned when the photographs were taken (the same lapels appear almost equally long in non-tilted views - Fig. 5A and 5F, respectively).

However, there are significant differences in soft part morphology. Whereas the shapes of the hemipenisoutline and of the left prehensile palps are fairly uniform in the different specimens (Figs 8A, C, E-F; $9 \mathrm{~A}, \mathrm{C}-\mathrm{D}, \mathrm{F})$, the second segment of the right prehensile palps ranges from elongated sub-triangular with almost equally rounded distal margin (Fig. 8B, D), to sub-rectangular with a clear blunt corner in this margin (Fig. 9B, E). It is not clear to what extend these differences are a biological reality, or whether the differences are distortions of the limbs caused by different positions in the slides. The differences are sufficiently small to be accepted as part of intra-specific variability, yet future investigations should take this variability into account. The morphology in the holotype (WAMC52228 - right prehensile palp in Fig. 8D) determines the specific morphology.

One male (WAMC52232 - Fig. 10A-D) had an aberrant morphology, with the terminal segment of the right prehensile palp (Fig. 10D) being even more elongated and with especially the terminal segment of the left prehensile palp being distally bilobed (Fig. 10C, C"), a morphology never before encountered in Cyprididae. Nevertheless the valves of this male show no differences with other type specimens (Fig. $5 \mathrm{~A}-\mathrm{C})$.

\section{Morphology within different clades/cryptic species}

Valve morphology of specimens for which molecular clades are known (A1: Fig. 6A-C; A2: Fig. 6DI; A3: Fig. 6J-L; A5: Fig. 6M-O, 7A-C) and for specimens from different populations for which no molecular data were available (Fig. 7D-N) again show no constant differences that could be used as identifying characters. There is some variability in size, shape and degree of crenulation of the lapel, but insufficiently so to use such features to characterise different clades/cryptic species.

One male specimen from BVT/10/02 and thus belonging to either cryptic species A1 or A3, had a right prehensile palp with a terminal segment clearly showing a blunt angle on the distal margin (Fig. 11C), while a male from BVT/10/06, and thus most likely belonging to clade A2, had a more elongated segment there with a more rounded distal margin (Fig. $11 \mathrm{G}$ ) as in the holotype. In both of these specimens, the terminal segment of the left prehensile palp is slightly shorter than in the type specimens (Fig. 11D, F). Hemipenis outlines (Fig. 11A-B, E) are indistinguishable from those in the types.

\section{Ecology and distribution}

Bennelongia timmsi sp. nov. is a typical rock pool species and occurs in fresh water in gnammas on various rocky outcrops in the south/central part of western Australia. Although it appears to be limited to this restricted area, it seems to be quite common there.

Bennelongia gnamma sp. nov.

Figs 12, 13A-E

urn:Isid:zoobank.org:act:6931D617-1443-4776-891B-C02752E6C0BE

\section{Diagnosis}

Valves in internal view (Fig. 12A-B, E-F) high, with greatest height situated in front of the middle; ventral margin almost evenly curved except for middle third. LV (Fig. 12A, E) with anterior il slightly overlapping. RV (Fig. 12B, F) with antero-ventral lapel subtriangular, asymmetrically produced with a dorsal point (Fig. 12H-K). 


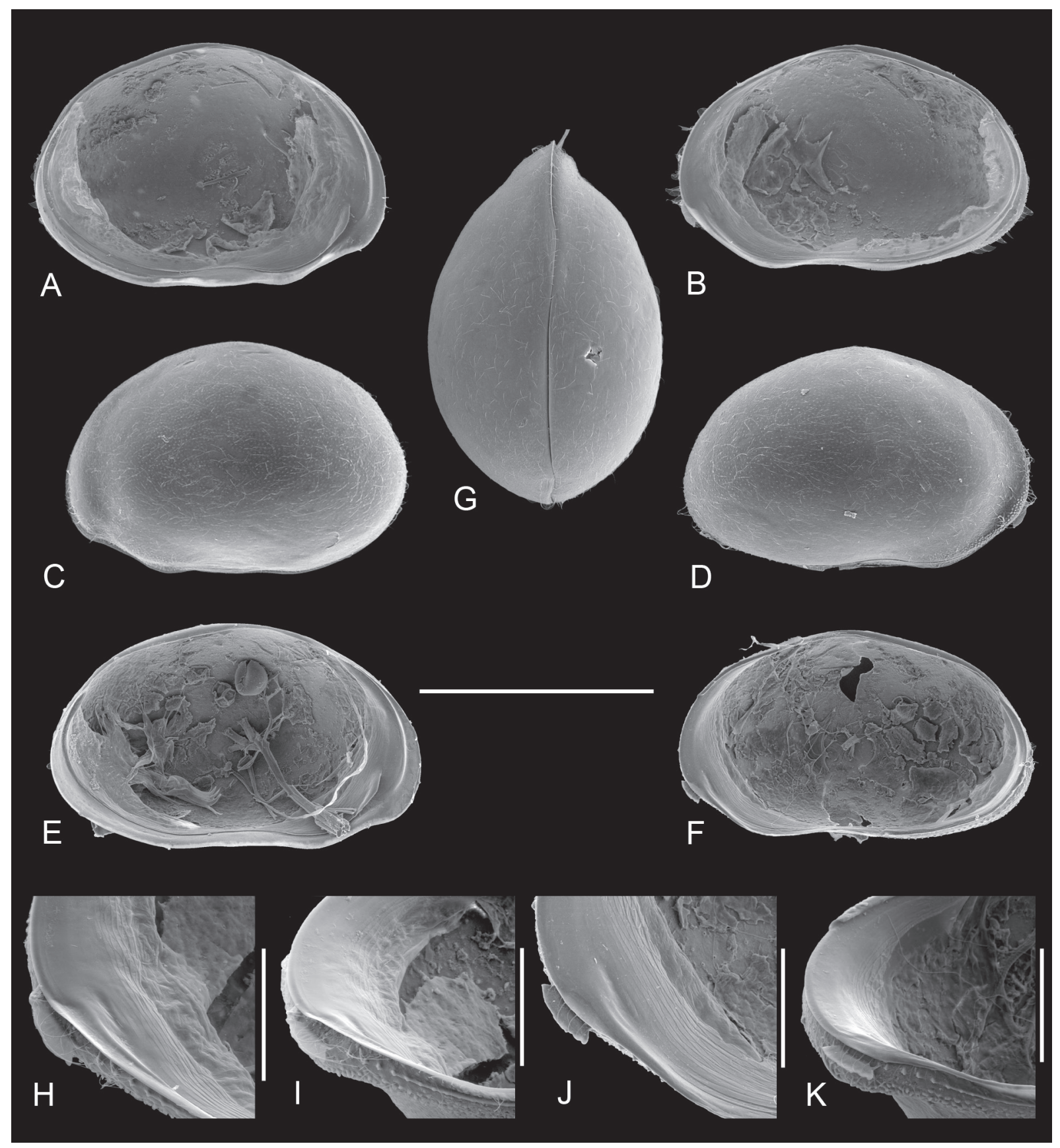

Fig. 12. Bennelongia gnamma sp. nov., type material from type locality (Cairn Rock, OSTR012A). A. + paratype, LVi (OC.3322). B. $q$ paratype, RVi (idem). C. $q$ paratype, LVe (idem). D. $q$ paratype, RVe (idem). E. $q$ holotype, LVi (WAMC52263). F. $q$ holotype, RVi (idem). G. $q$ paratype, CpD (WAMC52266). H. + paratype, RVi, detail anterior (OC.3322). I. क paratype, RVi, detail anterior, tilted (idem ). J. + holotype, RVi, detail anterior (WAMC52263). K. o holotype, RVi, detail anterior, tilted (idem). Scales: A-G $=1 \mathrm{~mm}$; H-K $=200 \mu \mathrm{m}$. 

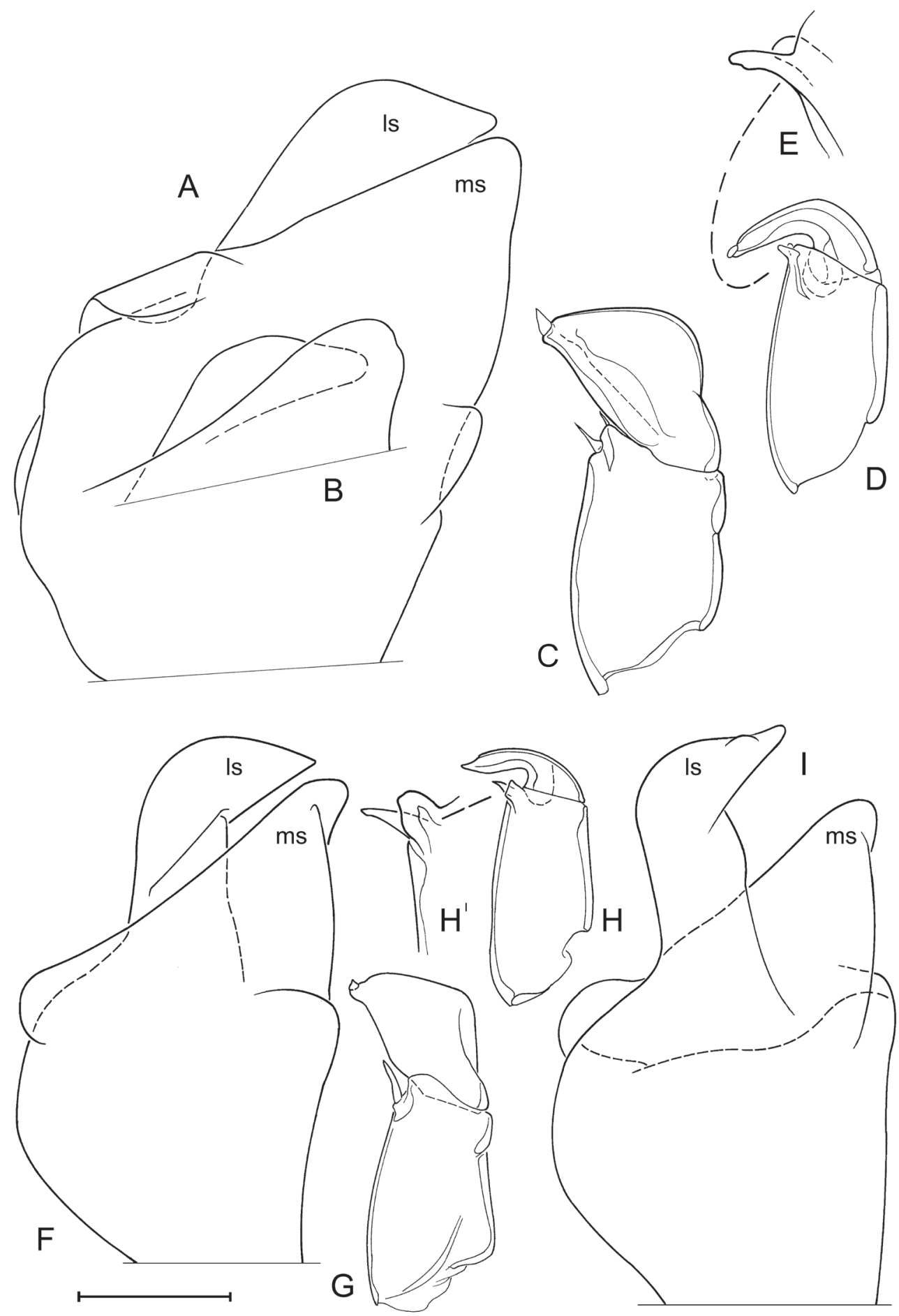

Fig. 13. Bennelongia gnamma sp. nov. (A-E, type specimens from type locality, Cairn Rock, OSTR012A) and Bennelongia hirsuta sp. nov. (F-I type specimens from type locality, Styles Rock, OSTR012D). Males. - A-E. B. gnamma sp. nov.: A. Hemipenis (both hemipenes symmetrical in this specimen, WAMC52264). B. Apical part of hemipenis (WAMC52265). C. Right prehensile palp (WAMC52264). D. Left prehensile palp (WAMC52264). E. Idem, detail of ventro-apical part of first segment (different specimen, WAMC52265). - F-I. B. hirsuta sp. nov. (WAMC52271): F. Hemipenis. G. Right prehensile palp. H. Left prehensile palp. H'. Idem, detail of ventro-apical part of first segment. I. Hemipenis. Scale: A-D, F-I $=92 \mu \mathrm{m} ; \mathrm{E}, \mathrm{H}^{\prime}=37 \mu \mathrm{m}$. 
Valves in external lateral view (Fig. 12C-D) high and rounded on all sides, even ventrally to some extent; hirsute and weakly pitted. Carapace in dorsal view (Fig. 12G) anteriorly with a mild rostrum.

Hemipenes (Fig. 13A) mostly symmetrical, with length of ls subequal to that of ms, in one specimen tip of ms even extending beyond that of 1s (Fig. 13B); ls with broad base, ventrally bluntly beak-shaped. Right prehensile palp (Fig. 13C) with distal segment broad, with anterior margin straight, distal margin bilobed. Left prehensile palp (Fig. 13D-E) with distal segment short, reaching beyond ventro-apical margin of proximal segment with less than half its length.

\section{Etymology}

The present species is named after the Australian term for small to middle-sized rock pools, namely gnammas, in which it occurs.

Measurements (all measurements in $\mu \mathrm{m}$ - see Table 1 for measurements of all specimens illustrated with SEM)

Holotype + (WAMC52264): RV: $\mathrm{L}=1550, \mathrm{H}=915 . \mathrm{LV}: \mathrm{L}=1620, \mathrm{H}=991$.

\section{Type locality}

Rock pools (gnammas) on Cairn Rock, WA, ca. $67 \mathrm{~km}$ SE of Merredin. Approximate coordinates: 3151'31" S, 118 50'39” E (WGS 84). All material (voucher sample OSTR012A; locality code SPS059) collected by J. McRae \& A. Pinder on 24 Sep. 1997 with a sweep net.

\section{Type material}

\section{Holotype}

o (WAMC52263), with soft parts dissected in a sealed slide and valves stored dry in a micropalaeontological slide.

\section{Allotype}

$\delta$ (WAMC52264), with soft parts dissected in a sealed slide, and valves stored dry in a micropalaeontological slide.

\section{Paratypes}

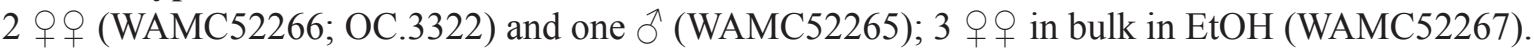

\section{Other material investigated}

? 1 (WAMC52268) from Yanneymooning Rocks (identification uncertain).

\section{Differential diagnosis}

Bennelonga gnamma sp. nov. can be distinguished from all other species in the lineage by the high and rounded shape of the valves and especially by the subequal ls and $\mathrm{ms}$ in the hemipenes.

\section{Ecology and distribution}

This species is thus far known with certainty only from its type locality, a set of rock pools on Cairn Rock. 


\author{
Bennelongia hirsuta sp. nov. \\ Figs 13F-I, 14 \\ urn:Isid:zoobank.org:act:CDE0D1E7-52E6-4F18-B6FF-B3AD48C6E4B4
}

\title{
Diagnosis
}

Valves elongated, with greatest height situated well in front of the middle, dorsal margin evenly sloping towards the posterior side; ventral margin anteriorly with pronounced mandibular curve. LV (Fig. 14A, D) with antero-ventral inner list large, well-overlapping the dorsal il. RV (Fig. 14B, E) with anteroventral lapel long, narrow and weakly crenulated (Fig. 14J-M).

Carapace in dorsal and ventral view (Fig. 14G-I) with greatest width in the middle, most hirsute, anteriorly with a clear rostrum; in lateral views (Fig. 14C, F) anteriorly with a clear LV $>$ RV overlap.

Hemipenes asymmetrical (Fig. 13F, I), ls with slender base, ventrally sharply beak-shaped and pointed, only slightly protruding beyond ventral tip of ms. Right prehensile palp (Fig. 13G) with distal segment stout and subquadrate, anterior margin straight, dorsal margin sinuous. Left prehensile palp (Fig. 13H, $\left.\mathrm{H}^{\prime}\right)$ with distal segment short and sickle-shaped, reaching beyond ventro-apical margin of proximal segment with less than a third of its length.

\section{Etymology}

Named after the hirsute ('hairy') nature of this species. All species of the B. barangaroo lineage are hirsute to some extent, but the present species is more so, with the entire carapace set with long and stiff setae.

Measurements (all measurements in $\mu \mathrm{m}$ - see Table 1 for measurements of all specimens illustrated with SEM)

Holotype $\widehat{O}$ (WAMC52269): RV: $\mathrm{L}=1260, \mathrm{H}=738 . \mathrm{LV}: \mathrm{L}=1320, \mathrm{H}=763$.

Allotype + (WAMC52270): RV: $\mathrm{L}=1400, \mathrm{H}=842 . \mathrm{LV}: \mathrm{L}=1470, \mathrm{H}=845$.

\section{Type locality}

Rock pools (gnammas) on Styles Rock, WA, ca. $80 \mathrm{~km} \mathrm{~N}$ of Esperance. Approximate coordinates:

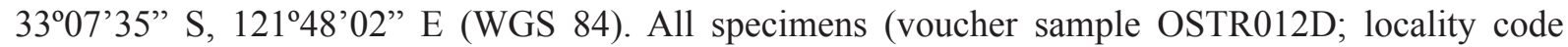
SPS139), collected on 07 Sep. 1998 by J. McRae \& A. Pinder with a sweep net.

\section{Type material}

\section{Holotype}

$\widehat{o}$ (WAMC52269), with soft parts dissected in a sealed slide and valves stored dry in a micropalaeontological slide.

\section{Allotype}

+ (WAMC52270), with soft parts dissected in a sealed slide, and valves stored dry in a micropalaeontological slide.

\section{Paratypes}

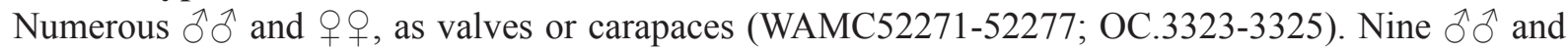
우 $ᄋ$ in bulk in $\mathrm{EtOH}$ (WAMC52278).

\section{Other material investigated}

? One + (WAMC52279) from Lilian Stokes Rocks (BVT/10/09) (identification uncertain). 


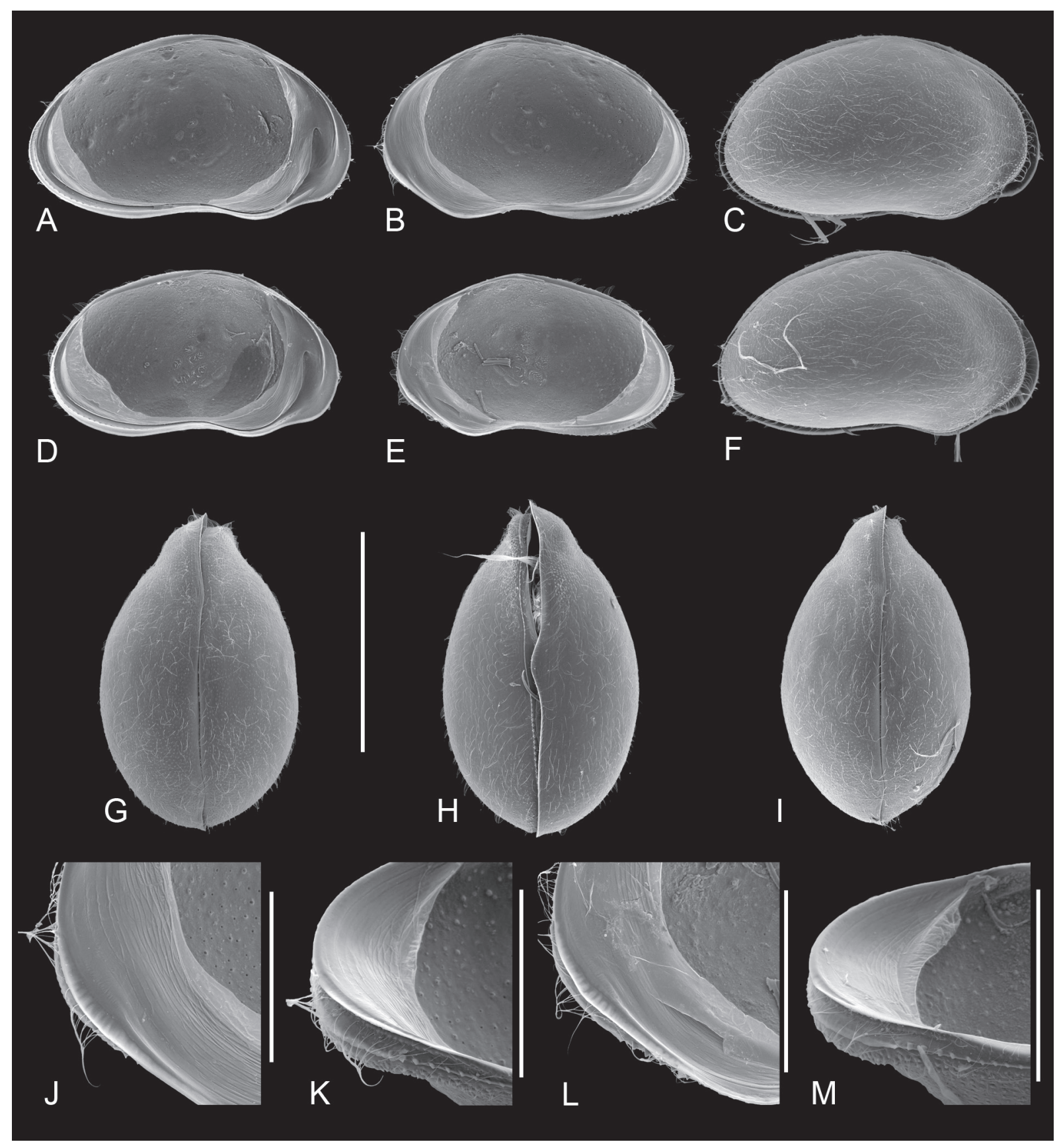

Fig. 14. Bennelongia hirsuta sp. nov., type specimens from type locality (Styles Rock, OSTR012D). A. $q$ allotype, LVi (WAMC52270). B. + allotype, RVi (idem). C. + paratype, CpRL (WAMC52277). D. $\delta^{\Uparrow}$

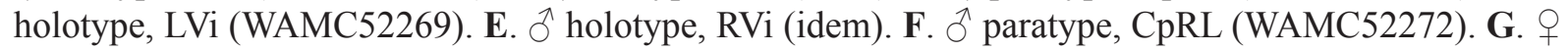
paratype, $\mathrm{CpD}$ (WAMC52275). H. 9 paratype, CpV (WAMC52276). I. ô paratype, CpD (WAMC52272).

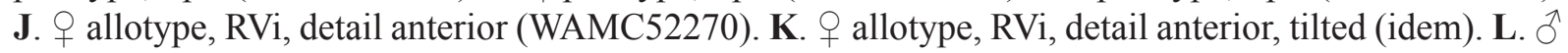

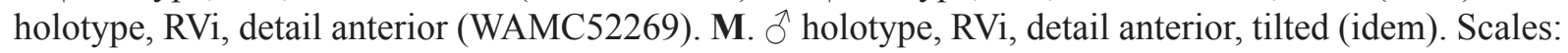
$\mathrm{A}-\mathrm{I}=1 \mathrm{~mm} ; \mathrm{J}-\mathrm{M}=300 \mu \mathrm{m}$. 


\section{Differential diagnosis}

Bennelongia hirsuta sp. nov. can be distinguished from all other species in the B. barangaroo lineage by the pointed shape of the ls in the hemipenis. Also the shapes of the distal segments of the prehensile palps are distinctive. In valve morphology, the species is easily recognisable by the long and narrow lapel on the RV (which is nevertheless very difficult to see with a normal binocular microscope) and the large antero-ventral il.

\section{Ecology and distribution}

The species is thus far only known with certainty from its type locality, a set of rock pools on Styles Rock.

Bennelongia ivanae sp. nov.

Fig. 15

urn:1sid:zoobank.org:act:2D60FC82-0938-475A-AF4E-E2DBE24D1D8D

\section{Diagnosis}

Valves (Fig. 15A-B) high and rounded, with greatest height situated well in front of the middle; dorsal margin with blunt angle towards the posterior side; ventral margin almost straight, without pronounced mandibular curve. LV (Fig. 15A) with antero-ventral il well-developed in lower third of the valve, dorsal il descending almost to ventral side, thus clearly overlapping with ventral il. RV (Fig. 15B) without antero-ventral lapel (Fig. 15H-J). Carapace in dorsal and ventral views (Fig. 15D-F) with greatest width in the middle, medium hirsute and slightly pitted, anteriorly with a hint of a rostrum; in lateral view (Fig. $15 \mathrm{C}, \mathrm{G})$ anteriorly with a clear LV $>$ RV overlap.

Male unknown.

\section{Etymology}

This species is named after Dr Ivana Karanovic (South Korea), in recognition of her contributions to the knowledge of the subterranean candonids of the Pilbara area.

Measurements (all measurements in $\mu \mathrm{m}$ - see Table 1 for measurements of all specimens illustrated with SEM)

Holotype + (WAMC52280): RV: $\mathrm{L}=1475, \mathrm{H}=915 . \mathrm{LV}: \mathrm{L}=1533, \mathrm{H}=948$.

\section{Type locality}

Rock pools (gnammas) on Holland Rocks, WA. Approximate coordinates: 33²1’35.66" S, $118^{\circ} 44^{\prime} 48.55^{\prime \prime}$ E (WGS 84) (sample DJC/02). All specimens collected and handpicked by D.J. Cale on 30 Aug. 2011.

\section{Type material}

\section{Holotype}

q valves stored dry (WAMC52280).

\section{Allotype}

As males are unknown, no allotype is designated.

\section{Paratypes}

Four $q+q$ with valves or carapaces stored dry (WAMC52281-52282; OC.3326-3327); $c a .25$ q $q$ stored dry as bulk in one micropalaeontological slide. 


\section{Other material investigated}

$?$ ? $q$ valves stored dry (WAMC52284) from Yanneymooning Rocks (OSTR013F).

\section{Differential diagnosis}

Bennelongia ivanae sp. nov. differs from all other WA species within the B. barangaroo lineage in the total absence of an antero-ventral lapel on the RV and in the fact that the antero-dorsal il in the LV runs almost entirely to the ventral margin. Bennelongia mckenziei Shearn et al., 2012 from Queensland also lacks the antero-ventral lapel on the RV completely, but it has a notably different valve and carapace
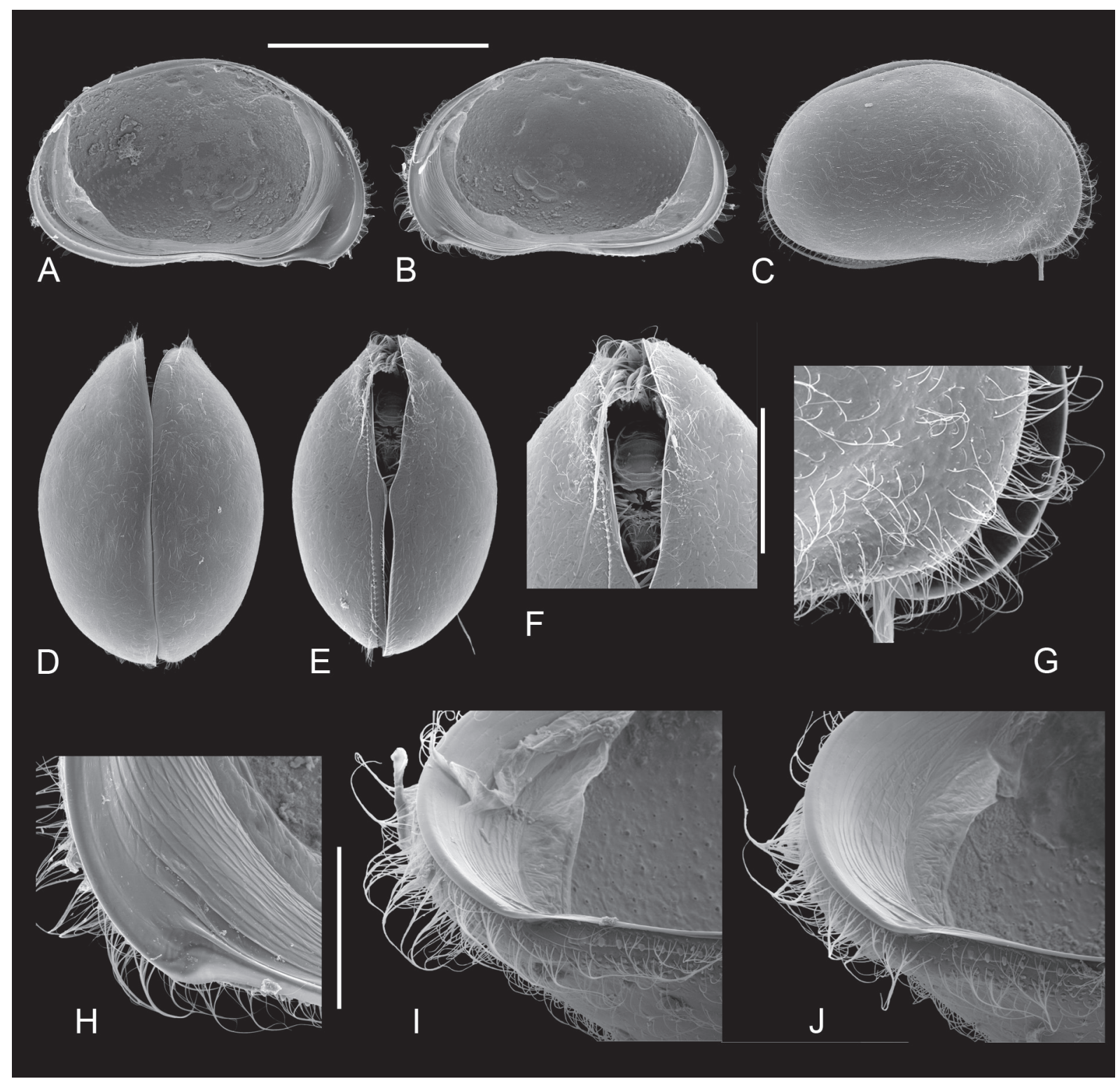

Fig. 15. Bennelongia ivanae sp. nov., type specimens from type locality (Holland Rocks, DJC/02). A. + holotype, LVi (WAMC52280). B. + holotype, RVi (idem). C. + paratype, CpRL (OC.3327). D. + paratype $\mathrm{CpD}$ (WAMC52281). E. + paratype, $\mathrm{CpV}$ (WAMC52282). F. + paratype, CpV, detail anterior (idem). G. + paratype, CpRL, detail anterior (OC.3327). H. + holotype, RVi, detail anterior (WAMC52280). I. + holotype, RVi, detail anterior, tilted (idem). J. + paratype, RVi, detail anterior, tilted (OC.3326). Scales: A-E $=1 \mathrm{~mm} ; \mathrm{F}=400 \mu \mathrm{m}$; G-J = $200 \mu \mathrm{m}$. 
shape, with a more pointed caudal margin and an evenly sloping dorsal margin in both valves, a shorter antero-dorsal il in the LV and an anterior LV $>\mathrm{RV}$ overlap in a carapace in right lateral view which is twice as large as in B. ivanae sp. nov.

\section{Remark}

Bennelongia ivanae sp. nov. and B. mckenziei have pronounced molecular differences, when the present sequences of $B$. ivanae sp. nov. are compared with those of Shearn et al. (2012). Because of the shorter lengths of the sequences provided by Shearn et al. (2012), the alignment of COI sequences from $B$. ivanae sp. nov. and B. mckenziei are not shown in the present paper.

\section{Ecology and distribution}

The species has thus far been found with certainty only from rockpools at Holland Rocks. Two tentatively identified females also originated from rock pools on another outcrop.

Bennelongia sp. nov. F2

\section{Material investigated}

Two $q$ ㅇ (KMWA.905, 906) in toto used for molecular screening, one $q$ with soft parts used for molecular screening and with broken RV stored dry in micropalaeontological cavity slide (WAMC52285 $=$ KMWA.904).

\section{Locality}

Lilian Stokes Rocks (eastern Wheatbelt - BVT/10/09), coordinates: 334'06” S, 12005'49” E. Collected on 25 Aug. 2010 by B.V. Timms.

\section{Remarks}

The specimens of the F2-group cluster close to those of B. ivanae sp. nov. in the phylogenetic tree (Fig. 2), but still constitute a separate genetic species (Table 2). A broken RV could be saved from only one of these specimens (KMWA.904), and it could be ascertained that the antero-ventral lapel on the RV is also fully absent (not shown). It is possible that cluster F2 will turn out to be a cryptic species within $B$. ivanae sp. nov., but this remains to be tested with new material. Note that the same sample (BVT/10/09) also contained one putative female of $B$. hirsuta sp. nov. (see above).

Bennelongia mcraeae sp. nov.

Figs 16-17

urn:1sid:zoobank.org:act:DFD1A720-E602-46CC-AABF-C6EDD63CD70E

\section{Diagnosis}

Valves (Fig. 16A-B, E-F) high and rounded, with greatest height situated on or close to the middle, dorsal margins almost evenly rounded; ventral margin weakly sinuous. LV (Fig. 16A, E) with anteroventral il large, reaching over half of the anterior margin; dorsal il descending along ca. 4/5 of anterior margin, both lists thus clearly overlapping. RV (Fig. 16B, F) with antero-ventral lapel fairly ventrally inserted, large and pronounced, with rounded, weakly crenulated margin (Fig. 16D, I-L). Carapace in lateral view (Fig. 16C- D) pitted, especially along anterior and posterior margins, and set with few setae.

Hemipenes (Fig. 17A, F) largely symmetrical, 1s with broad base, ventrally bluntly beak-shaped, only protruding significantly beyond ventral tip of ms. Right prehensile palp (Fig. 17C, E) with distal segment stout, but of somewhat variable shape, either strongly subquadrate or with rounded dorsal and anterior 

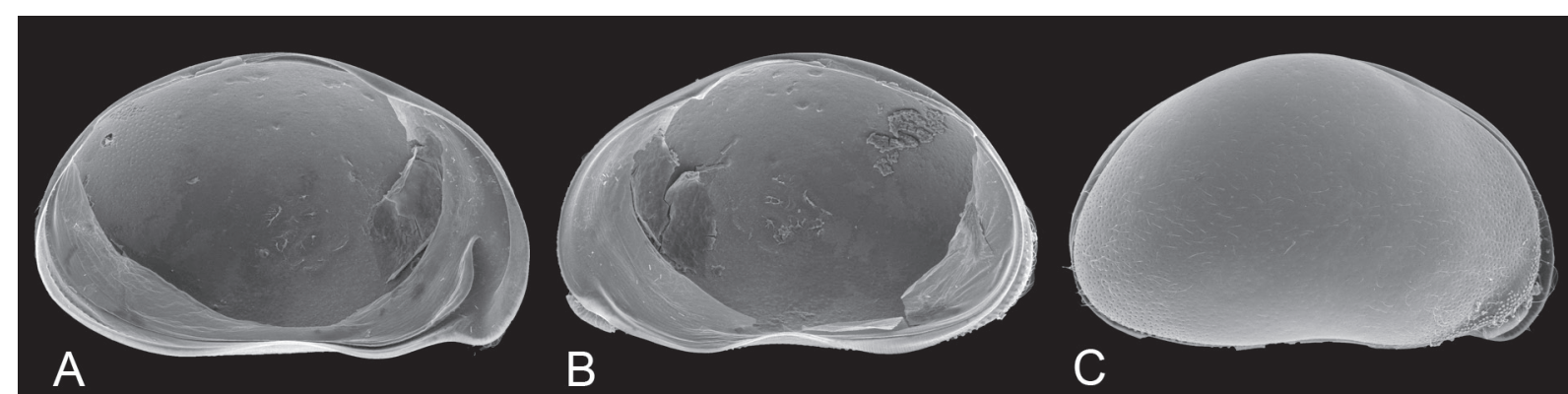

A

B

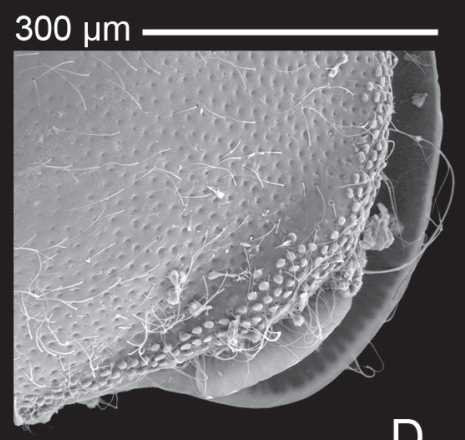

D

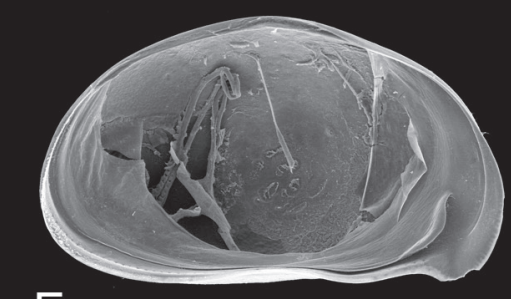

E
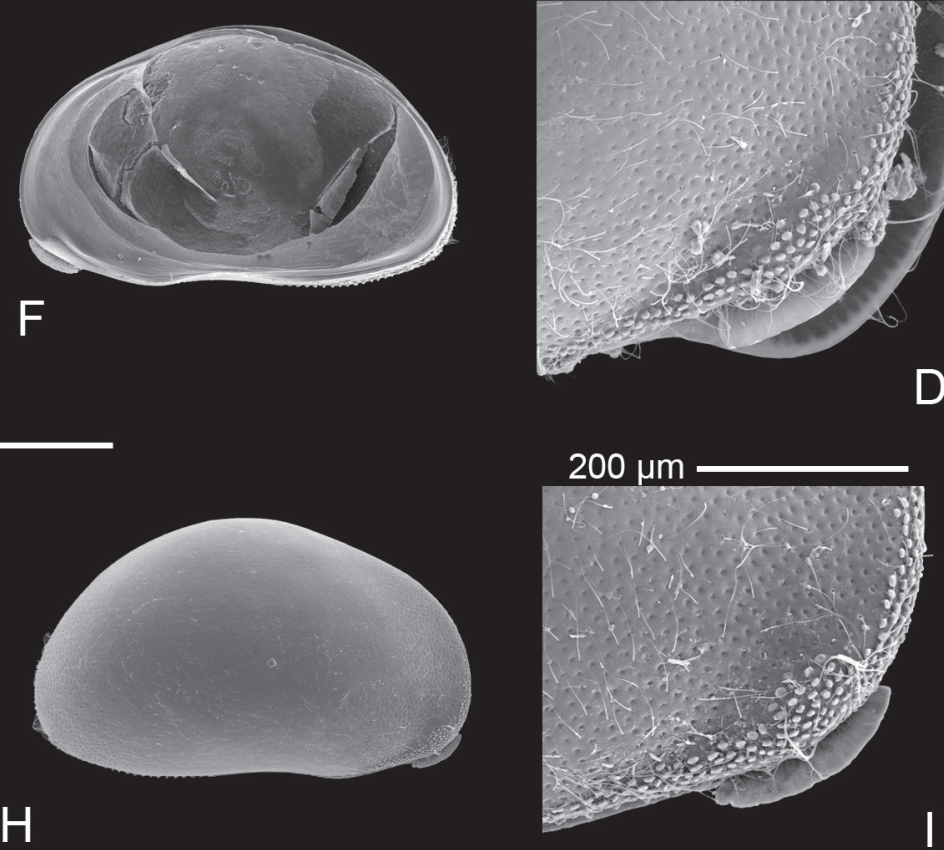

G

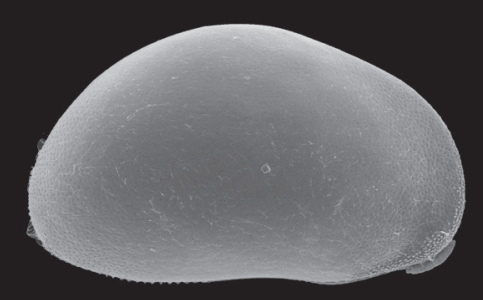

$\mathrm{H}$

I
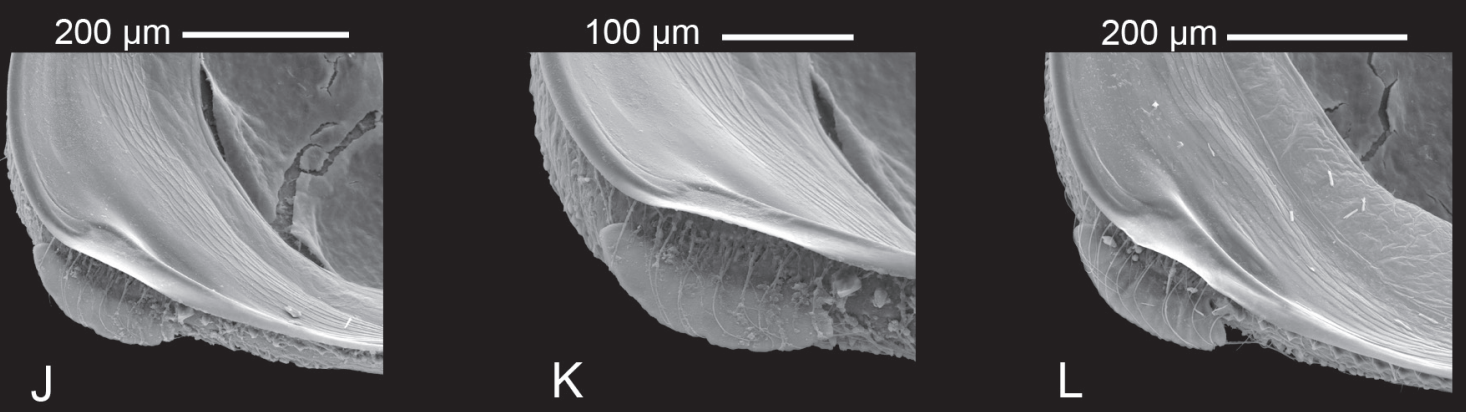

Fig. 16. Bennelongia mcraeae sp. nov., type specimens from type locality (Arro Lake, OSTR014). A. ㅇ allotype, LVi (WAMC52287). B. $q$ allotype, RVi (idem). C. + paratype, CpRL (WAMC52289). D. +9 paratype, CpRL, detail anterior (idem). E. $\overbrace{}^{\Uparrow}$ holotype, LVi (WAMC52286). F. $\overbrace{}^{\Uparrow}$ holotype, RVi (Idem). G. $\widehat{\delta}$ holotype, LVe (idem). H. $\widehat{\partial}$ holotype, RVe (idem). I. $\widehat{\jmath}$ holotype, RVe, detail anterior (idem). J.

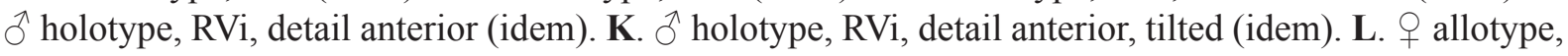
$\mathrm{RVi}$, detail anterior, tilted (WAMC52287). Scales $=1 \mathrm{~mm}$ unless otherwise indicated. 

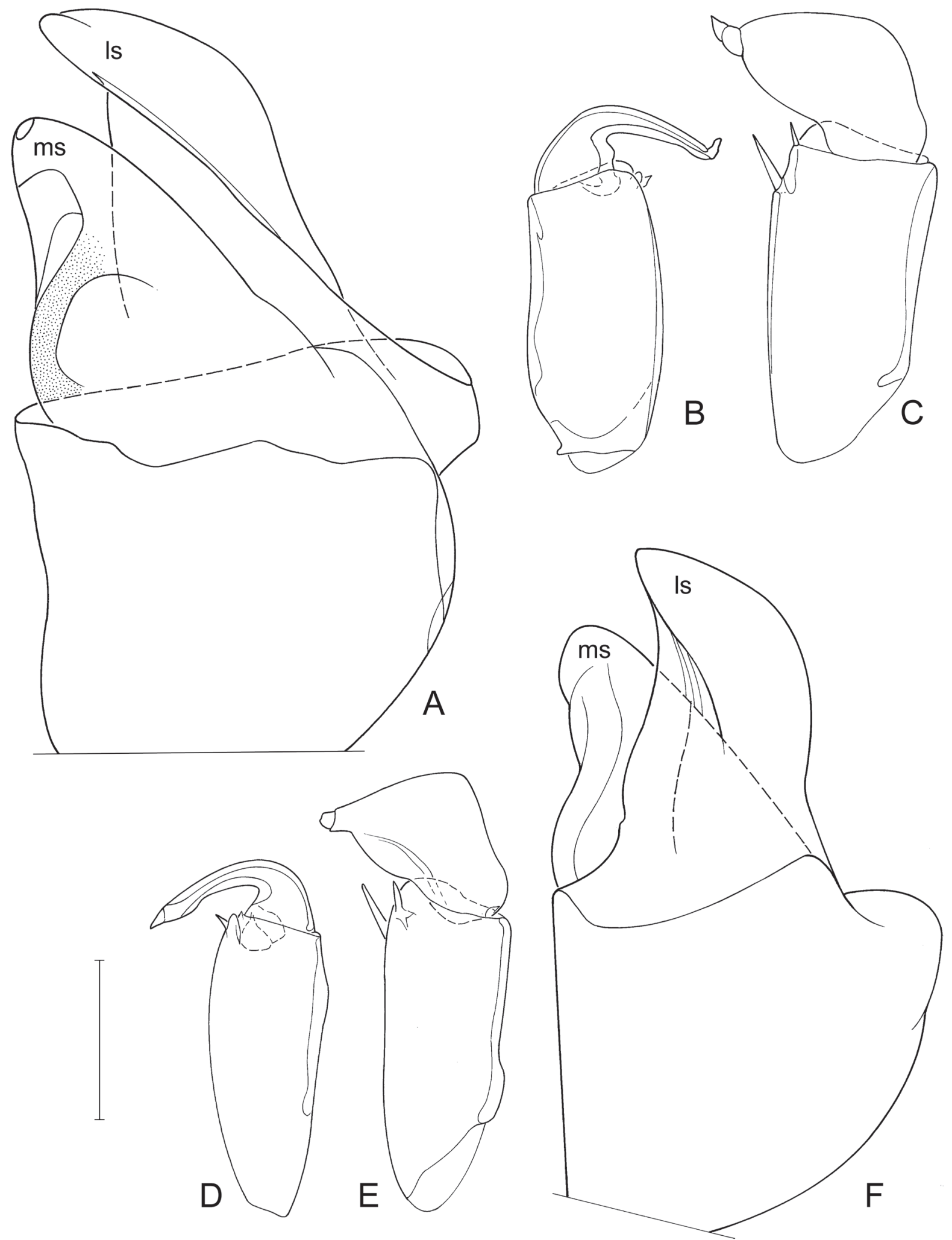

Fig. 17. Bennelongia mcraeae sp. nov., type males from type locality (Arro Lake, OSTR014B). - A-C. Holotype $\widehat{~}$ (WAMC52286). A. Hemipenis. B. Left prehensile palp. C. Right prehensile palp. - D-F. Paratype $\widehat{\sigma}$ (OC.3328). D. Left prehensile palp. E. Right prehensile palp. F. Hemipenis. Scale: A-F = $92 \mu \mathrm{m}$. 
margins. Left prehensile palp (Fig. 17B, D) with distal segment long and slender, reaching beyond ventro-apical margin of proximal segment with at least half of its length.

\section{Etymology}

The species is named in honour of Jane McRae (Perth, WA) in acknowledgement of her vast knowledge of the taxonomy and morphology of many invertebrate groups of Western Australia. She also collected the type material of the present species and has unrelentingly provided technical help towards the present revision of Bennelongia since 2006.

Measurements (all measurements in $\mu \mathrm{m}$ - see Table 1 for measurements of all specimens illustrated with SEM)

Holotype $\widehat{O}$ (WAMC52286): RV: $\mathrm{L}=1480, \mathrm{H}=878 . \mathrm{LV}: \mathrm{L}=1560, \mathrm{H}=896$.

Allotype + (WAMC52287): RV: $\mathrm{L}=1608, \mathrm{H}=1002 . \mathrm{LV}: \mathrm{L}=1663, \mathrm{H}=1033$.

\section{Type locality}

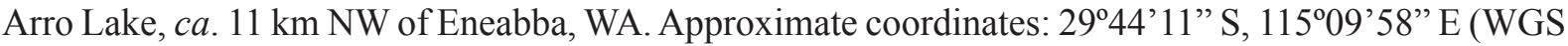
84). All specimens collected by J. McRae \& A. Pinder (voucher OST14B; locality code SPS182) on 23 Sep. 1999 with a sweep net. Arro Lake is an open lake with a Melaleuca/Casuarina fringe. Water chemistry at the time of collecting: Salinity $=0.15 \mathrm{~g} / \mathrm{l}, \mathrm{pH}=7.32$. Nutrient levels were fairly high: total $\mathrm{N}=1700 \mu \mathrm{g} / 1$; total $\mathrm{P}=220 \mu \mathrm{g} / \mathrm{l}$. The milky-white colour of the water equates to a high level of turbidity (2200 NTU).

\section{Type material}

\section{Holotype}

$\widehat{\jmath}$ (WAMC52286), with soft parts dissected in a sealed slide and valves stored dry in a micropalaeontological slide.

\section{Allotype}

ㅇ (WAMC52287), with soft parts dissected in a sealed slide, and valves stored dry in a micropalaeontological slide.

\section{Paratypes}

Two $\widehat{\jmath}$ (dissection: OC.3328; valves: WAMC52288) and one + carapace (WAMC52289). Three females in bulk in EtOH (WAMC52290).

\section{Differential diagnosis}

The species is characterised especially by the large and stout lapel on the RV but also by the large anteroventral il on the LV.

\section{Ecology and distribution}

Lake Arro ( $c a .300 \mathrm{~km} \mathrm{~N}$ of Perth) is a large flat-bottomed body of water with episodic inflow that holds water for about 4-24 months after inflow. The lake has a clay base and sediment-driven turbidity. This species is known only from the type locality. From the same sample as the one that yielded B. macraeae sp. nov., Timms (2002) described a new species of Anostraca, Branchinella complexidigitata Timms, 2002. 


\section{Bennelongia scanloni sp. nov. \\ Figs $18-20$ \\ urn:1sid:zoobank.org:act:B41BF127-BEEC-47CE-A687-FACD6CBCF028}

\section{Diagnosis}

Valves (Fig. 18A-B, D-E) high, with greatest height situated close to the middle, dorsal margin evenly sloping towards the posterior side; ventral margin sinuous. LV (Fig. 18A, D) with antero-ventral il of medium size, covering lower third of valve, antero-dorsal il descending to about halfway along anteroventral il. RV (Fig. 18B, E) with antero-ventral lapel tear-shaped in untilted lateral view; in tilted view, lapel subtriangular with rounded ventral point (Fig. 18K-N). Carapace in dorsal and ventral views (Fig. 18G-J) with greatest width in the middle, hirsute and pitted, anteriorly with a clear rostrum; in right lateral view (Fig. 18C, F) with large anterior LV $>$ RV overlap, anterior margins of RV and LV not parallel.

Hemipenes (holotype: Fig. 20F) asymmetrical, 1s with broad base, ventrally bluntly pointed (more so in one hemipenis than in the other), largely protruding beyond ventral tip of ms. Right prehensile palp (holotype: Fig. 20D) with distal segment stout and subquadrate, with sharp angle between anterior and dorsal margins, both of these margins almost straight. Left prehensile palp (holotype: Fig. 20E) with distal segment sickle-shaped and of intermediate length, reaching beyond ventro-apical margin of proximal segment with about half of its length.

\section{Etymology}

The species is named in honour of Mike Scanlon (Perth, WA) in acknowledgement of his unrelenting technical help since 2006 towards the present revision of Bennelongia.

Measurements (all measurements in $\mu \mathrm{m}$ - see Table 1 for measurements of all specimens illustrated with SEM)

Holotype $\widehat{~}$ (WAMC52291): RV: $\mathrm{L}=1223, \mathrm{H}=694 . \mathrm{LV}: \mathrm{L}=1294, \mathrm{H}=714$.

Allotype + (WAMC52292): RV: $\mathrm{L}=1263, \mathrm{H}=752 . \mathrm{LV}: \mathrm{L}=1356, \mathrm{H}=775$.

\section{Type locality}

One Tree Hill Creek, ca. $62 \mathrm{~km}$ SE of Dongara, WA. Approximate coordinates: $29^{\circ} 35^{\prime} 19.0^{\prime \prime} \mathrm{S}$, $15^{\circ} 26^{\prime} 31.0$ " E (WGS 84). All specimens (sample DJC/11; locality code SPS180) collected by D.J. Cale on 10 Sep. 2011 with a sweep net. Water chemistry at time of collecting: K25 $5.62 \mathrm{mS} / \mathrm{cm}$, pH 6.68, water temperature $20.8^{\circ} \mathrm{C}$.

\section{Type material}

\section{Holotype}

$\delta$ (WAMC52291), with soft parts dissected in a sealed slide and valves stored dry in a micropalaeontological slide.

\section{Allotype}

q (WAMC52292) valves stored dry in a micropalaeontological slide.

\section{Paratypes}

Numerous males and females either as dissection, or as valves or carapaces stored dry (WAMC5229352304; OC.3329-3331). Ca. $30 \widehat{\partial} \widehat{\partial}$ and 9 우 stored as bulk in EtOH (WAMC52305). 


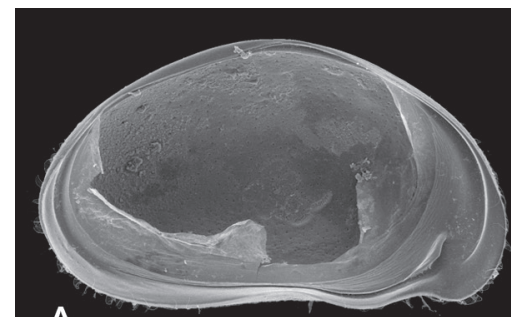

A
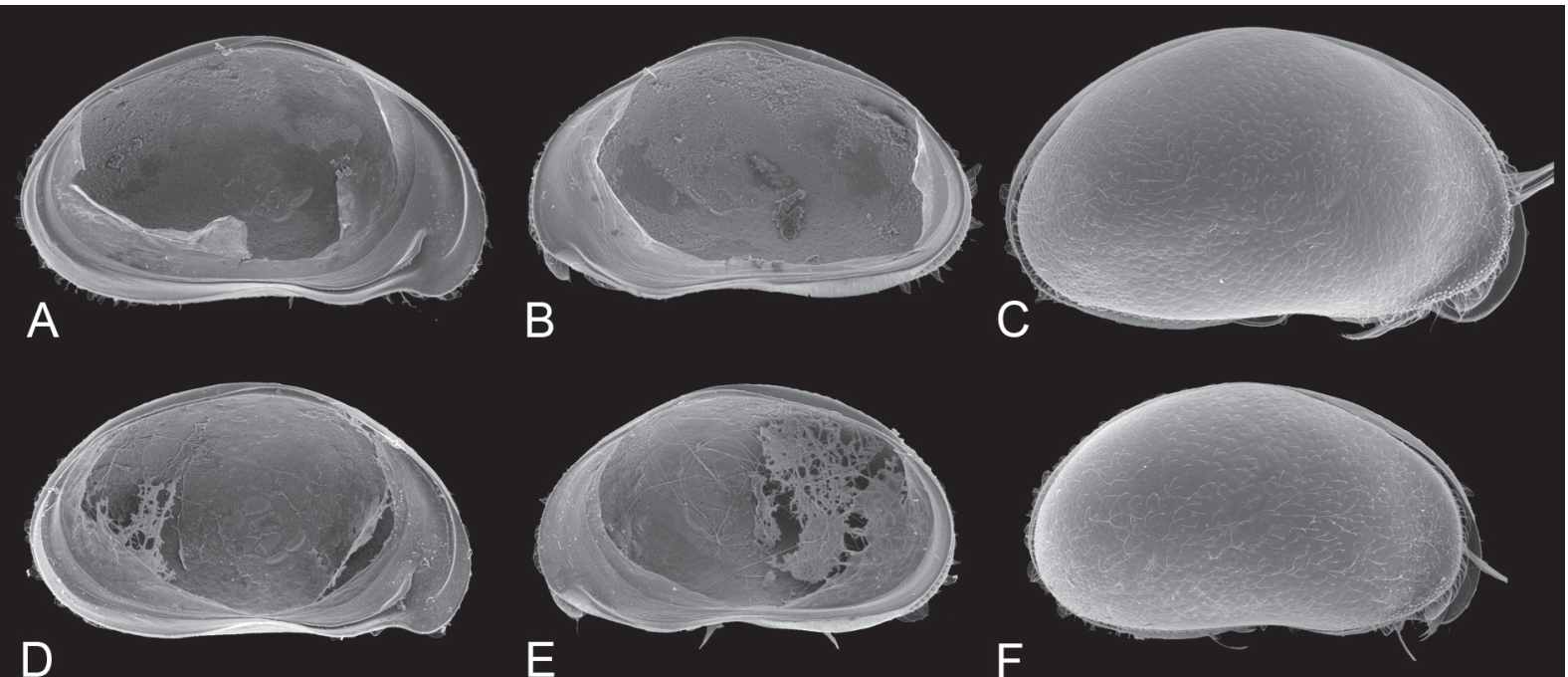

$\mathrm{D}$

$\mathrm{E}$

$\mathrm{F}$
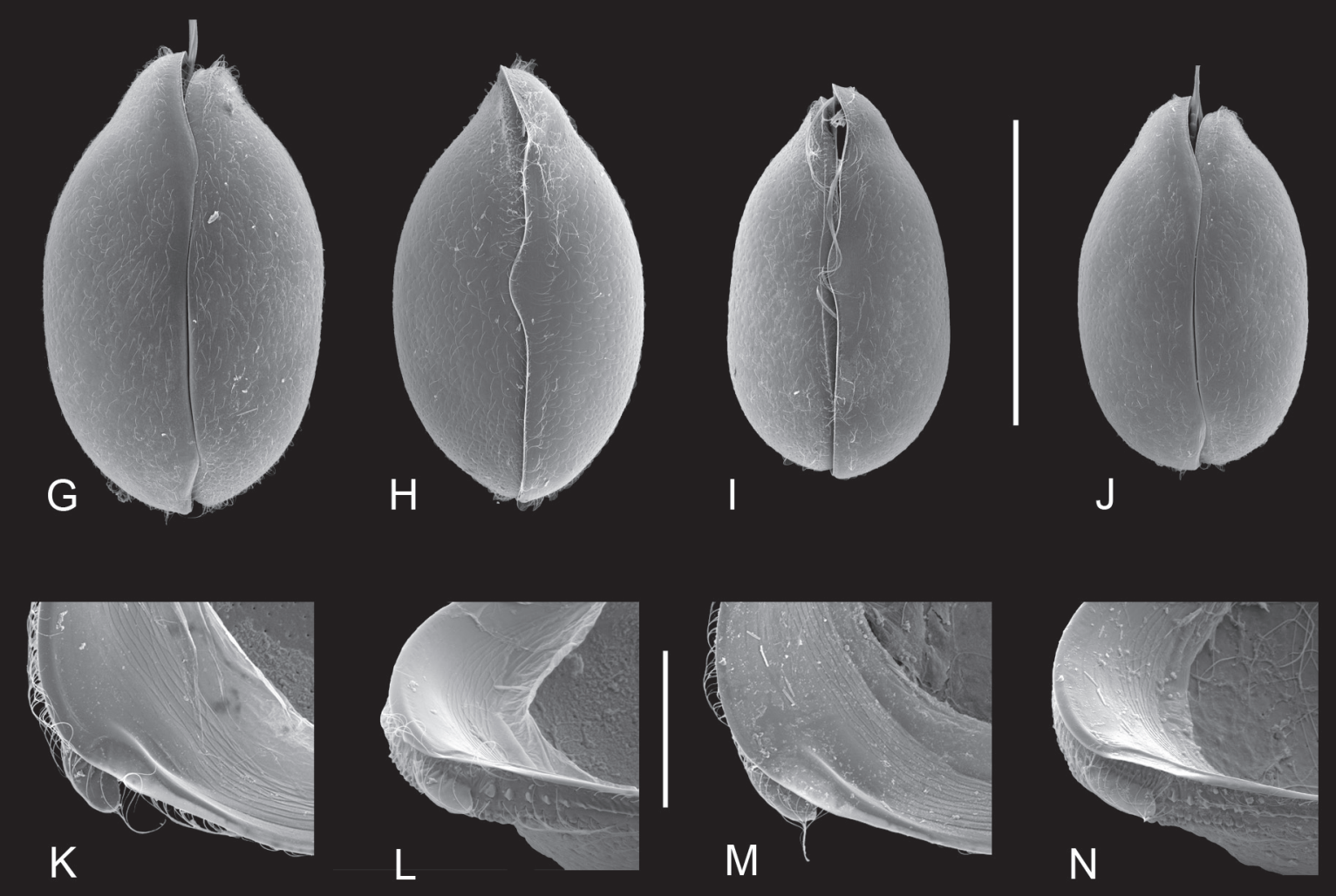

Fig. 18. Bennelongia scanloni sp. nov., type specimens from type locality (One Tree Hill Creek, DJC/11). A. $q$ allotype, LVi (WAMC52292). B. $q$ allotype, RVi (idem). C. $q$ paratype, CpRL (WAMC52299). D. ô paratype, LVi (OC.3329). E. ô paratype, RVi (idem). F. ô paratype, CpRL (WAMC52295). G. $ᄋ$ paratype, CpD (WAMC52297). H. क paratype, CpV (WAMC52298). I. ô paratype, CpV (WAMC52295). J. § paratype, CpD (WAMC52296). K. क allotype, RVi, detail anterior (WAMC52292). L. + allotype, $\mathrm{RVi}$, detail anterior, tilted (idem). M. ô paratype, RVi, detail anterior (OC.3329). N. § paratype, RVi, detail anterior, tilted (idem). Scales: A-J = $1 \mathrm{~mm} ; \mathrm{K}-\mathrm{N}=200 \mu \mathrm{m}$. 


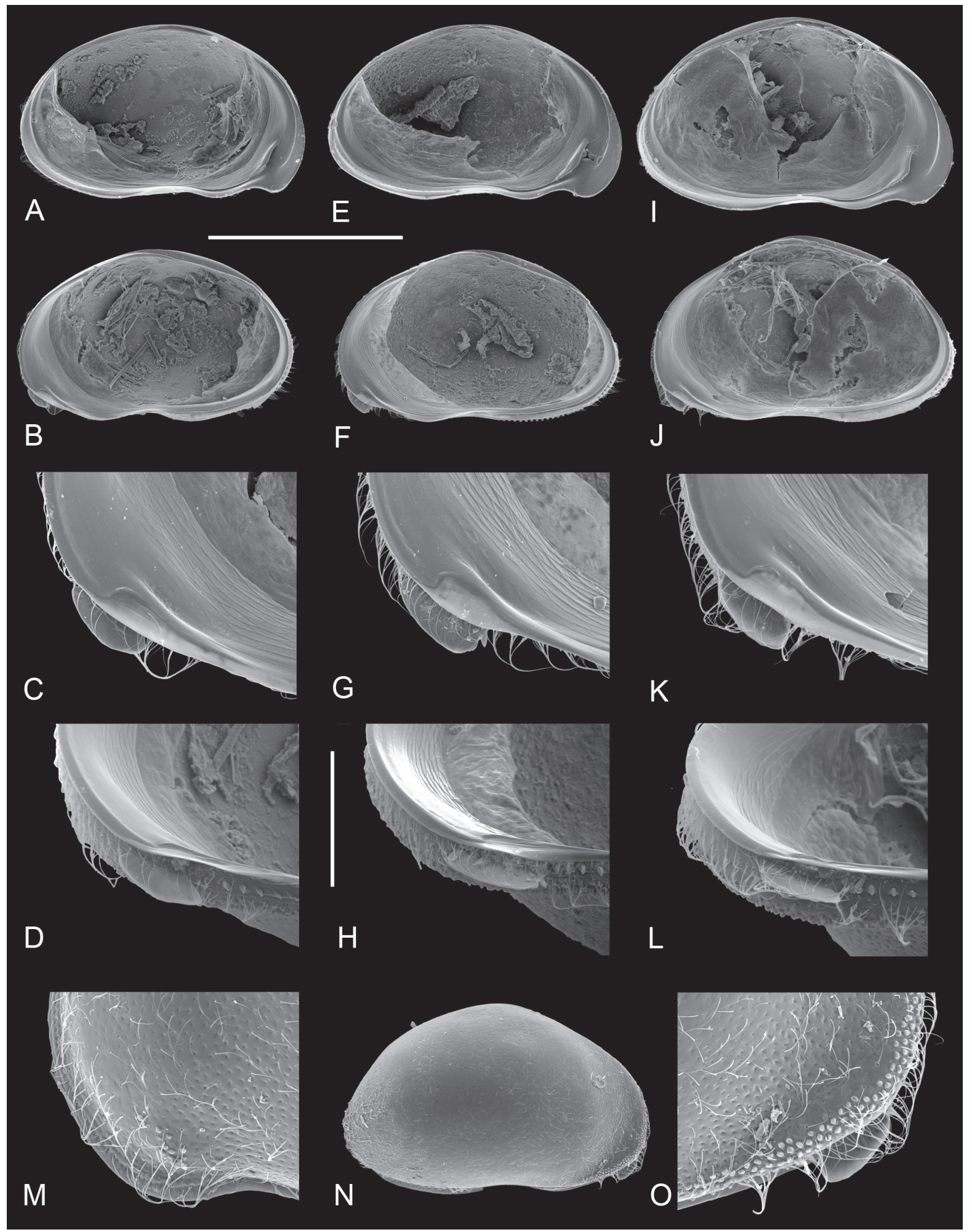

Fig. 19. Bennelongia scanloni sp. nov., non-type specimens. - A-D. Pool at Latham-Coorow Rd (BVT/10/10, +, WAMC52307)). A. LVi. B. RVi. C. RVi, detail anterior. D. RVi, detail anterior, tilted. - E-H. Tin Dog Creek (DJC/19, q, OC.3335). E. LVi. F. RVi. G. RVi, detail anterior. H. RVi, detail anterior, tilted. - I-O. Pool at Brookton Hwy (Warrine Park) (DJC/23, o, OC.3337). I. LVi. J. RVi. K. RVi, detail anterior. L. RVi, detail anterior, tilted. M. LVe, detail anterior. N. RVe. O. RVe, detail anterior. Scales: A-B, E-F, I-J, N = 1 mm; C-D, G-H, K-M, O = $200 \mu \mathrm{m}$. 


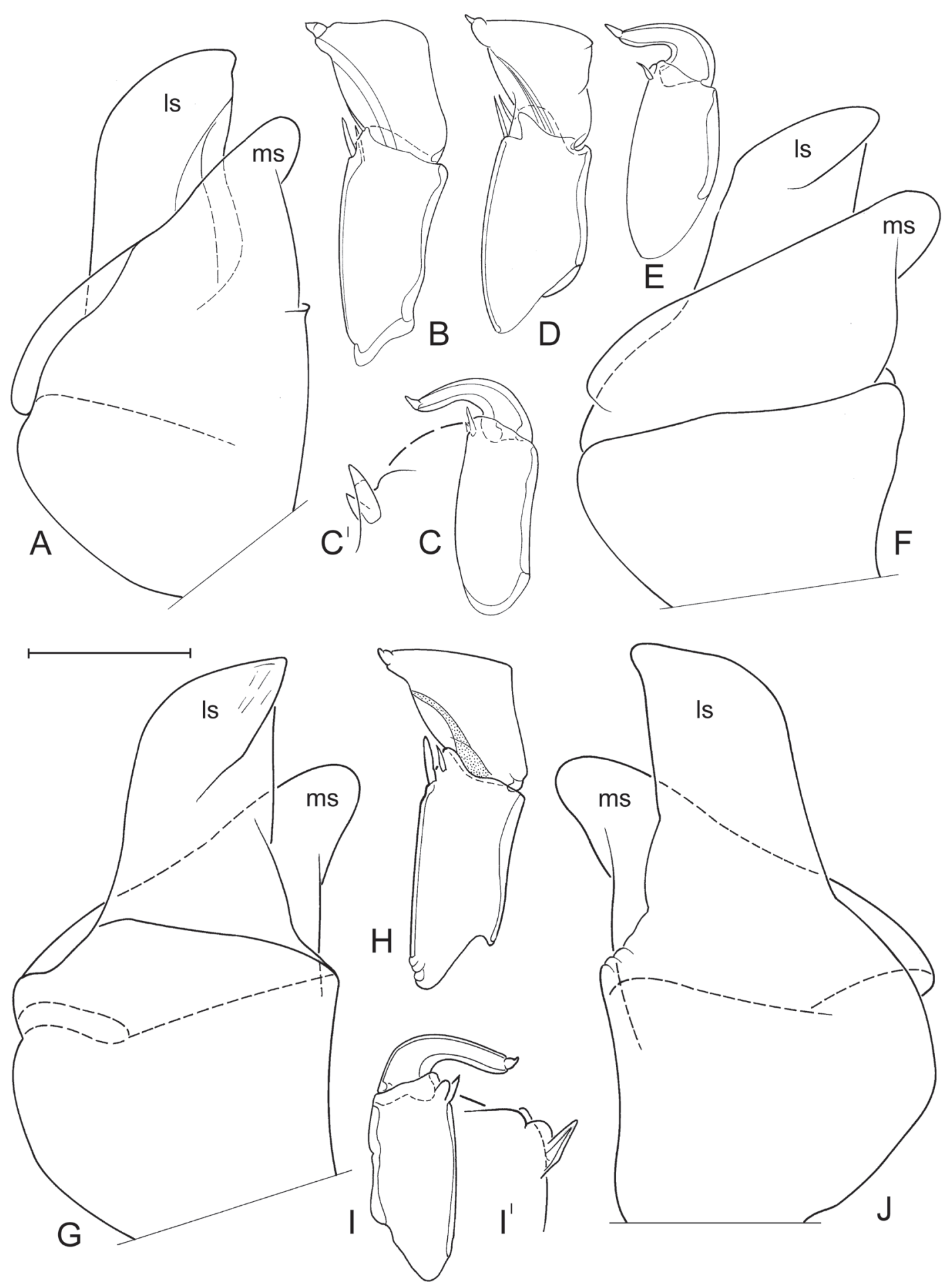

Fig. 20. Bennelongia scanloni sp. nov., males. - A-C'. Non-type (OC.3338, Three Springs Tumulus Stream - TST): A. Hemipenis (both hemipenes symmetrical in this specimen). B. Right prehensile palp. C. Left prehensile palp. C'. Idem, detail of ventro-apical part of first segment. - D-F. Holotype (WAMC52291, One Tree Hill Creek - DJC/11): D. Right prehensile palp. E. Left prehensile palp. F. Hemipenis (both hemipenes symmetrical in this specimen). - G-J. Non-type (WAMC52327, from OSTR013C): G. Hemipenis. H. Right prehensile palp. I. Left prehensile palp. I'. Idem, detail of ventroapical part of first segment. J. Hemipenis. Scale: A-J $=92 \mu \mathrm{m}$; C', I' $=37 \mu \mathrm{m}$. 
MARTENS K., HALSE S. \& SCHÖN I., The Bennelongia barangaroo lineage in Western Australia

\section{Other material investigated}

One Tree Hill Creek. 29³5'19.0” S, 115'26'31.0” E, collected by S. Halse and A. Pinder on 11 Aug. 1999 (OSTR013C), see Fig. 20G-J (WAMC52327). Water chemistry at time of collecting: K25 $3.12 \mathrm{mS} /$ $\mathrm{cm}, \mathrm{pH} 7.65$, water temperature $19.4^{\circ} \mathrm{C}$.

Pools at Latham-Coorow Rd. 29 $51^{\prime}$ 'S, 116 ${ }^{\circ} 16^{\prime}$ E (sample BVT/10/10), collected by B.V. Timms on 10 Sep. 2010 (WAMC52306-52308; OC.3332-3333).

Dam at Solomons Well. Approximate coordinates: 31 ${ }^{\circ} 11^{\prime} 58.8^{\prime \prime}$ S, 116 $21^{\circ}$ '47.7” E (sample DJC/04), collected by D.J.Cale on 09 Sep. 2011 (4 + $ᄋ$ : WAMC52332-52334; OC.3343). Water chemistry at time of collecting: $\mathrm{K} 250.12 \mathrm{mS} / \mathrm{cm}, \mathrm{pH} 7.85$, water temperature $14.4^{\circ} \mathrm{C}$.

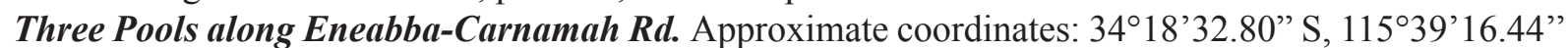
E (sample DJC/09), collected by D.J. Cale on 10 Sep. 2011 (2 o o : WAM52310; OC.3334). Water chemistry at time of collecting: $\mathrm{K} 258.7 \mathrm{mS} / \mathrm{cm}, \mathrm{pH} 7.2$, water temperature $20.3^{\circ} \mathrm{C}$.

Second pool along Carnamah-Eneabba Road on south side (Eneabba Springs). Approximate coordinates: $29^{\circ} 48^{\prime} 23.62^{\prime \prime}$ S, $115^{\circ} 25^{\prime} 6.11^{\prime \prime}$ E (sample DJC/10), collected by D.J. Cale on 10 Sep. 2011. Water chemistry at time of collecting: $\mathrm{K} 253.19 \mathrm{mS} / \mathrm{cm}, \mathrm{pH} 6.6$, water temperature $20.9^{\circ} \mathrm{C}$.

Petruder Dam. Approximate coordinates: $30^{\circ} 25^{\prime} 20.87^{\prime}$ ' S, $116^{\circ} 57^{\prime} 39.43^{\prime \prime}$ E (sample DJC/15), collected by D.J. Cale on 11 Sep. 2011 (5 q : : WAMC52329-52331, OC.3341-3342). Water chemistry at time of collecting: K25 $0.16 \mathrm{mS} / \mathrm{cm}, \mathrm{pH} 7.85$, water temperature $22.0^{\circ} \mathrm{C}$.

Tin Dog Creek. Approximate coordinates: $31^{\circ} 11^{\prime} 53.5^{\prime \prime} \mathrm{S}, 117^{\circ} 01^{\prime} 41.4^{\prime \prime}$ E (sample DJC/19), collected by D.J. Cale on 23 Sep. 2011 (우: WAMC52311-52315, OC.3335-3336. Juveniles: WAMC5231652318). Water chemistry at time of collecting: $\mathrm{K} 251.56 \mathrm{mS} / \mathrm{cm}, \mathrm{pH} 7.06$, water temperature $24.2^{\circ} \mathrm{C}$.

Pools near Brookton Hwy (in Warrine Park). Approximate coordinates: 32 23'50.4” S, 116 48'00.4" E (sample DJC/23), collected by D.J. Cale on 01 Oct. 2011 (4 q 9 : WAMC52319-52321, OC.3337). Water chemistry at time of collecting: $\mathrm{K} 250.44 \mathrm{mS} / \mathrm{cm}, \mathrm{pH} 8.1$, water temperature $21.6^{\circ} \mathrm{C}$.

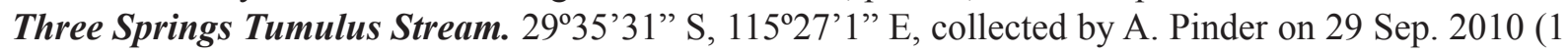
male: OC.3338; 2 q + : WAMC52322-52323).

East Lake Bryde. 33⒉ ' S, 118 49' E (sample BRYDE7), collected by D.J. Cale on 22 Mar. 2006 (4 우: WAMC52324-52326; OC.3339). Water chemistry at time of collecting: $\mathrm{K} 250.17 \mathrm{mS} / \mathrm{cm}, \mathrm{pH} 6.91$, water temperature $25.8^{\circ} \mathrm{C}$.

Lake Cronin. Episodically filled waterbody with extensive shrub and Melaleuca fringe, collected by S. Halse and A. Pinder on 25 Sep. 1997. Approximate coordinates: $32^{\circ} 23^{\prime} 02^{\prime \prime}$ S, $119^{\circ} 45^{\prime} 51^{\prime \prime}$ E. Water chemistry at time of collecting: K25 $0.23 \mathrm{mS} / \mathrm{cm}, \mathrm{pH} 9.48$, water temperature $18.0^{\circ} \mathrm{C}$. Material investigated: one dissected 9 ( $\mathrm{nr}$ OS.544), with soft parts in a sealed slide and valves stored dry in micropalaeontological cavity slide (illustrated: Fig. 24E-H).

Reserve Esperance 26140 near Munglinup. Seasonally filled lake with trees across most of flooded area, collected 27 Oct. 1986 by S. Halse. Approximate coordinates: 33²6’24" S, 120³1'48” E. Water chemistry: salinity $0.27 \mathrm{mg} / \mathrm{L}$ TDS, pH 6.93. Material investigated: one dissected $q$ (nr OS.604), with soft parts in a sealed slide and valves stored dry in a micropalaeontological cavity slide (illustrated: Fig. 25I-L).

Remarks on the latter two localities: the lapels of both specimens are slightly larger than in most specimens of B. scanloni sp. nov. and as (1) no males are at hand to check for the morphology of the hemipenes and the prehensile palps in these populations and (2) no molecular data are available, the identifications of these two specimens are tentative.

\section{Additional notes on cryptic species}

Specimens from the type locality (One Tree Hill Creek, sample DJC/11) all belong to cryptic species $\mathrm{E} 1$, which is thus the true B. scanloni sp. nov. s.s. Also specimens from sample DJC/23 (pools near Brookton Hwy in Warrine Park) belong to this lineage (Fig. 19I-O). Two specimens from cryptic species 
B2 are also illustrated here, one female from pools beside Latham-Coorow Rd (western Wheatbelt) (BVT/10/10) (Fig. 19A-D) and one female from Tin Dog Creek (DJC/19) (Fig. 19E-H). There are no clear differences between the two cryptic species in valve morphology.

The soft parts of the male from Three Springs Tumulus Stream (TST) (Fig. 20A-C) are slightly different from those of the holotype (Fig. 20D-F). Yet, within the molecular phylogeny the TST specimens cluster closely with the cryptic species E1, which is the same as for the type specimens. The hemipenis outline and the prehensile palps of the male from OSTR013C (Fig. 20G-J) are almost identical to those of the holotype, though no molecular data on this population are available, and it is thus also not clear to which of the two cryptic species within $B$ scanloni sp. nov. this specimen belongs.

Thus far, the two molecular species cannot be distinguished morphologically. Interestingly, these two cryptic lineages occur sympatrically in no less than 4 localities (DJC/09, DJC/11, DJC/19 and BVT/10/10).

\section{Differential diagnosis}

The drop-shaped lapel on the RV and the sharp angle on the distal margin of the terminal segment of the right prehensile palp distinguish $B$. scanloni sp. nov. from all other species within the B. barangaroo lineage.

\section{Ecology and distribution}

This is arguably the most common species in this lineage in the south-western part of WA. It typically occurs in pools, dams and lakes with soft sediments.

Bennelongia calei sp. nov.

Figs 21-22

urn:Isid:zoobank.org:act:DBD2498B-9E05-4E2A-9597-67844A85653E

Bennelongia barangaroo - De Deckker 1981a: 104, fig. 9 (partim).

Diagnosis (based on type specimens)

Valves (Fig. 21A-B, E-F) elongated, with greatest height situated close to the middle, dorsal margin evenly sloping towards the posterior side; ventral margin almost straight. LV (Fig. 21A, E) with anteroventral il large and reaching beyond middle of valve, antero-dorsal il descending to about halfway along antero-ventral il. RV (Fig. 21B, F) with antero-ventral lapel large, elongated and wide, with crenulated edge (Fig. 21C-D, G-H). Carapace in dorsal and ventral views (Fig. 21K-L) with greatest width in the middle, most slender of all species described here, external surface rather smooth to weakly pitted, set with only few short setae; anteriorly with a clearly delimited rostrum. Carapace in right lateral view (Fig. 21I-J) with greatest height in the middle, dorsal margin evenly sloping to bluntly rounded posterior margin; anteriorly with the widest $\mathrm{LV}>\mathrm{RV}$ overlap of all species described here.

Males unknown.

\section{Etymology}

This species is named after D.J. Cale (Woodvale, WA) in honour of his longstanding contribution to the knowledge of freshwater invertebrates in WA, including at Fraser Lake which is the type locality of the present species (Cale et al. 2004), and also in recognition of the fact that he has collected so many of the samples used for the present revision of the Bennelongia barangaroo lineage. 
MARTENS K., HALSE S. \& SCHÖN I., The Bennelongia barangaroo lineage in Western Australia

Measurements (all measurements in $\mu \mathrm{m}$ - see Table 1 for measurements of all specimens illustrated with SEM)

Holotype क (WAMC52335): RV: $\mathrm{L}=1480, \mathrm{H}=857 . \mathrm{LV}: \mathrm{L}=1555, \mathrm{H}=865$.

\section{Type locality}

Fraser Lake, ca. $8 \mathrm{~km}$ SE of Dowerin, WA. Approximate coordinates: 31 $1^{\circ} 15^{\prime} 18.0^{\prime \prime} \mathrm{S}, 117^{\circ} 4^{\prime} 22.0^{\prime \prime} \mathrm{E}$ (WGS 84). All material (sample code DJC/18) collected on 23 Sep. 2011 by D.J. Cale with a sweep net. Water chemistry at time of collecting: $\mathrm{K} 251.76 \mathrm{mS} / \mathrm{cm}, \mathrm{pH} 8.1$, water temperature $23.9^{\circ} \mathrm{C}$.

\section{Type material}

\section{Holotype}

o (WAMC52335) valves stored dry in a micropalaeontological slide.

\section{Allotype}

As males are unknown, no allotype is designated.

\section{Paratypes}

Eight 우, either as dried valves or carapaces (WAMC52337-52341; OC.3344-3345). Ca. 45 females stored as bulk in EtOH (WAMC52342).

\section{Other material investigated}

Fraser Lake (type locality). Four $ᄋ+9$ valves and carapaces stored dry (WAMC52353-52356) from the same locality, but collected on another date (sample nr SPM017B, collected by D.J. Cale, 24 Nov. 2000), were also used during the present assessment of this species but are not considered as type material here. Second pool along Carnamah-Eneabba Road on south side (Eneabba Springs). Approximate coordinates: $29^{\circ} 48^{\prime} 23.62^{\prime \prime} \mathrm{S}, 15^{\circ} 25^{\prime} 6.11^{\prime \prime} \mathrm{E}$ (sample DJC/10), collected by D.J. Cale on 10 Sep. 2011 (one + WAMC52349). Water chemistry at time of collecting: K25 $3.19 \mathrm{mS} / \mathrm{cm}, \mathrm{pH}$ 6.6, water temperature $20.9^{\circ} \mathrm{C}$.

Jerramungup West. Approximate coordinates: 3359'16.03” S, 118 56'28.15” E (sample DJC/36), collected by D.J. Cale on 21 Oct. 2011 (five $ㅇ ㅜ$ valves and carapaces stored dry WAMC52350-52352; OC.3348-3349). Water chemistry at time of collecting: $\mathrm{K} 250.73 \mathrm{mS} / \mathrm{cm}, \mathrm{pH} 8.74$, water temperature $25.8^{\circ} \mathrm{C}$

Oak Flat W pit gnamma via Goomalling. Approximate coordinates $31^{\circ} 08^{\prime} 21^{\prime \prime} \mathrm{S}, 116^{\circ} 52^{\prime} 46^{\prime \prime} \mathrm{E}$ (sample BVT/11/04), collected by B.V. Timms on 16 Aug. 2011 (four 우 9 valves and carapaces stored dry WAMC52343-52344; OC.3346-3347).

Horse Collar gnamma, on Magee Rd via Kulin. Approximate coordinates: 3248'04" S, 118²3'34" E (sample BVT/11/05), collected by B.V. Timms on 4 Sep. 2011 (four $q$ ㅇ $~ v a l v e s$ and carapaces stored dry WAMC52345-52348).

\section{Additional illustrations}

Several other populations of this species were found and for four of these (listed above), valves of female specimens are also illustrated here (Fig. 22). All of these specimens comply with the above diagnosis, and where specimens were available for molecular analyses, they also fell into the B. calei sp. nov. - cluster. No cryptic species were identified in this species.

\section{Differential diagnosis}

Bennelongia calei sp. nov. can easily be distinguished from all other species in the B. barangaroo lineage by the elongated and stout antero-ventral lapel on the RV, which is slightly rounded and has a 


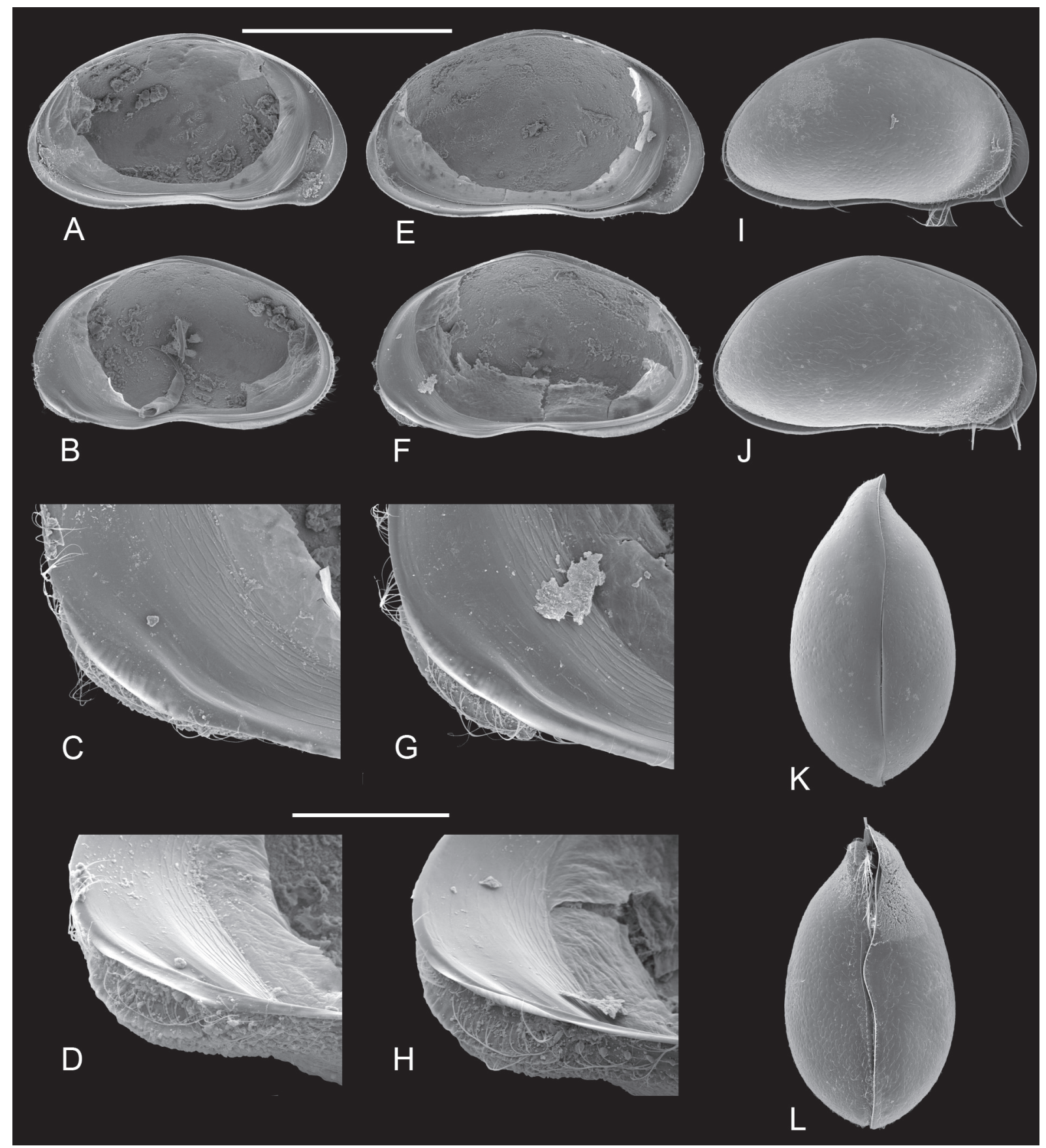

Fig. 21. Bennelongia calei sp. nov. - A-H, J-L. Fraser Lake (type locality, females, DJC/18): A. Paratype, LVi (OC.3344). B. Paratype, RVi (idem). C. Paratype, RVI, detail anterior (idem). D. Paratype, RVI, detail anterior, tilted (idem). E. Holotype, LVi (WAMC52335). F. Holotype, RVI (idem). G. Holotype, RVi, detail anterior (idem). H. Holotype, RVi, detail anterior, tilted (idem). J. Paratype, CpRL (OC.3345). K. Paratype, CpD (WAMC52338). L. Paratype, CpV (WAMC52337). - I. Oak Flat W pit gnamma, via Goomalling (BVT/11/04, non-type female, WAMC52343). CpRL. Scales: A-B, E-F, I-L = $1 \mathrm{~mm}$; C-D, G-H = $200 \mu \mathrm{m}$. 

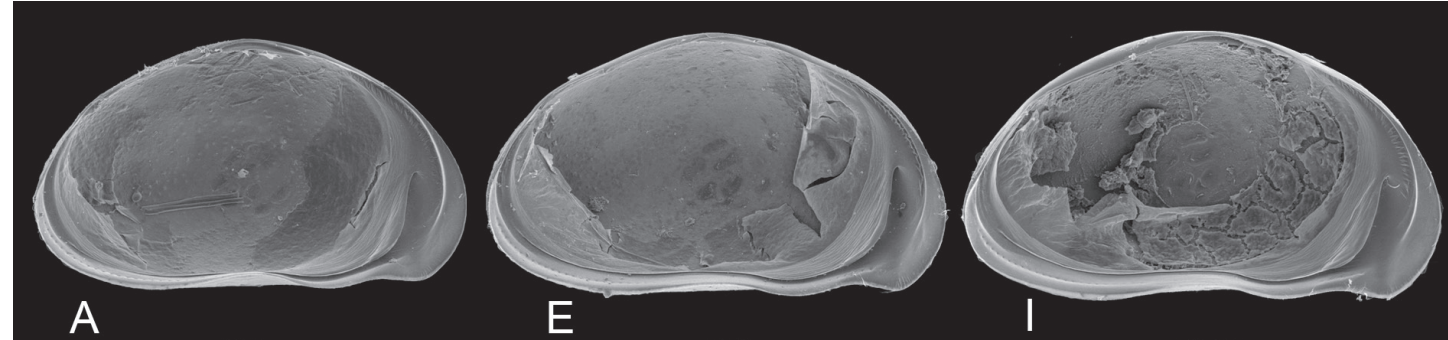

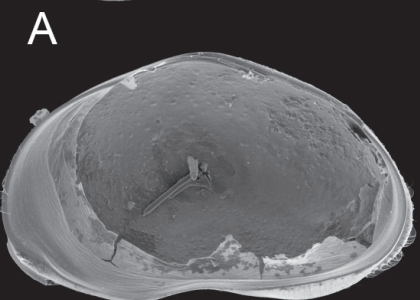

B
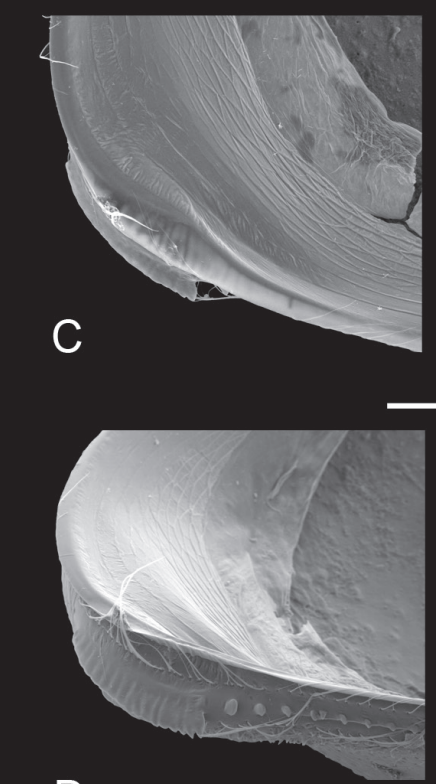

D

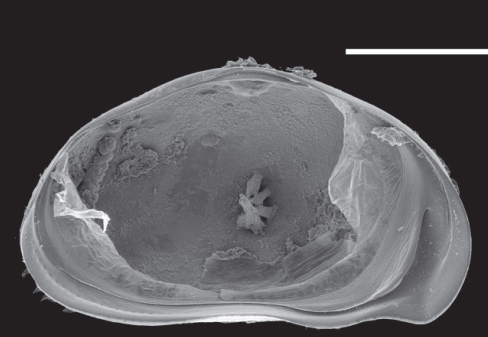

$\mathrm{M}$

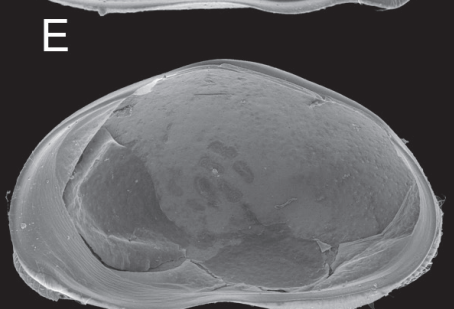

$\mathrm{F}$

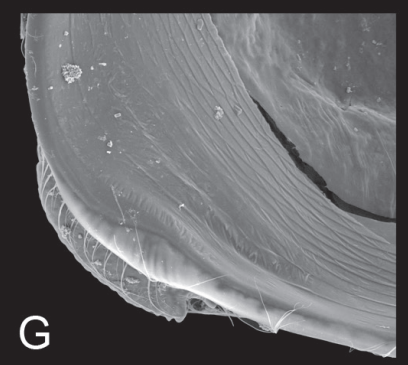

G

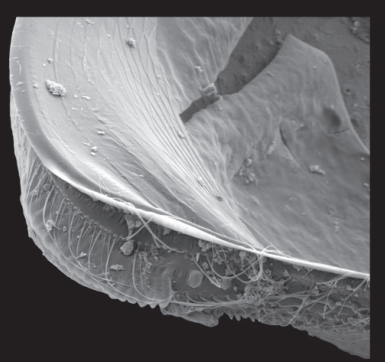

$\mathrm{H}$

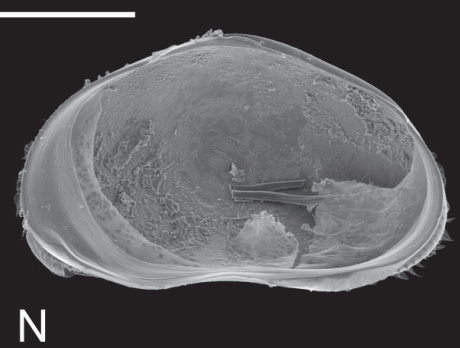

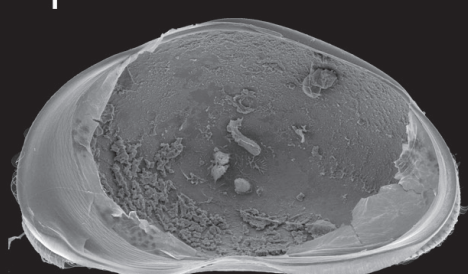

$\mathrm{J}$
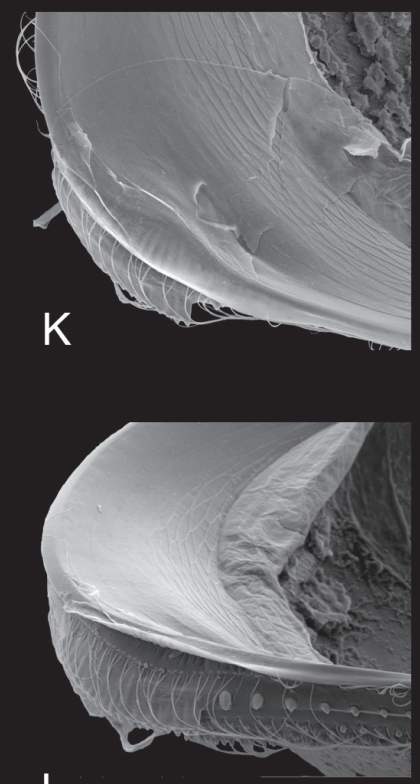

$\mathrm{L}$

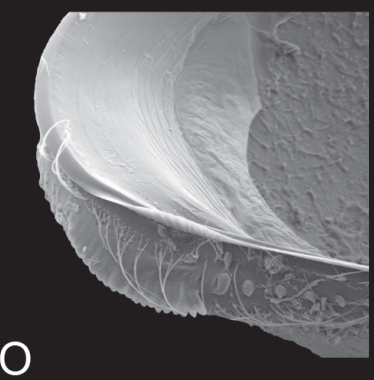

Fig. 22. Bennelongia calei sp. nov., non-type specimens. - A-D. Oak Flat W pit gnamma, via Goomalling

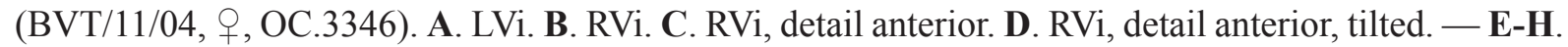
Horse Collar gnamma, on Magee Rd via Kulin (BVT/11/05, +, WAMC52345). E. LVi. F. RVi. G. RVi, detail anterior. H. RVi, detail anterior, tilted. - I-L. Second pool along Carnamah-Eneabba Road on south side, Eneabba Springs (DJC/10, क, WAMC52349). I. LVi. J. RVi. K. RVi, detail anterior. L. RVi, detail anterior, tilted. - M-O. Pool at Jerramungup West (DJC/36, + , OC.3348). M. LVi. N. RVi. O. RVi, detail anterior, tilted. Scales: A-B, E-F, I-J, M-N $=1 \mathrm{~mm}$; C-D, G-H, K-L, O $=200 \mu \mathrm{m}$. 
crenulated distal margin, the large anterior LV $>$ RV overlap, the stout antero-ventral il on the LV and the clear anterior rostrum on the carapace in dorsal or ventral view.

\section{Ecology and distribution}

The species is most common in pools and lakes in the southwest of WA. However, B. calei sp. nov. was also recovered from a totally different kind of habitat, namely pit gnammas near Goomalling (BVT/11/04) and Kulin (BVT/11/05). Whereas the other species in this lineage apparently occur either in rock pools or in soft bottomed pools and lakes, $B$. calei sp. nov. can apparently survive in both (very different) types of habitats. Bennelongia calei sp. nov. is, together with $B$. timmsi sp. nov. and $B$. scanloni sp. nov., one of the more common species in its area.

Bennelongia dedeckkeri Shearn et al., 2012

Figs 23, 24I-L

Bennelongia dedeckkeri sp. nov. - Shearn et al., 2012: 10-14, figs 4-5.

\section{Material investigated}

Dam at Kylena Well (Pilbara). Approximate coordinates: 22 06'00” S, 119³9’00" E (sample KIES10). Collected on 23 Apr. 2006 by the authors.

Unnamed saline billabong $\mathbf{N}$ of Coolcalaya Rd (Murchinson, Gascoyne). Approximate coordinates: $27^{\circ} 48^{\prime} 28^{\prime \prime}$ S, 114² $48^{\prime} 18^{\prime \prime}$ E (sample SIKE2). Collected on 5 Jul. 2011 by the authors. Water chemistry at time of collecting: $\mathrm{K} 258.8 \mathrm{mS} / \mathrm{cm}, \mathrm{pH} 8.8$, water temperature $11.0^{\circ} \mathrm{C}$.

McNeil Claypan, Carnarvon (Murchinson, Gascoyne). Approximate coordinates: 245'06" S, $113^{\circ} 42^{\prime} 56^{\prime \prime}$ E (sample SIKE9). Collected on 6 Jul. 2011 by the authors. Water chemistry at time of collecting: K25 $0.19 \mathrm{mS} / \mathrm{cm}, \mathrm{pH} 9.4$, water temperature $10.8^{\circ} \mathrm{C}$.

Roadside ditch 1, North-West Coastal Hwy, Minilya Station (Murchinson, Gascoyne). Approximate coordinates: $23^{\circ} 54^{\prime} 25^{\prime}$ S, $114^{\circ} 01^{\prime} 45^{\prime \prime}$ E (sample SIKE18). Collected on 7 Jul. 2011 by the authors. Water chemistry at time of collecting: K25 $0.66 \mathrm{mS} / \mathrm{cm}, \mathrm{pH} 7.4$, water temperature $17.3^{\circ} \mathrm{C}$.

Roadside ditch 2, North-West Coastal Hwy, (Murchinson, Gascoyne). Approximate coordinates: $23^{\circ} 54^{\prime} 25^{\prime}$ " S, $114^{\circ} 01^{\prime} 47^{\prime \prime}$ E (sample SIKE19). Collected on 7 Jul. 2011 by the authors. Water chemistry at time of collecting: $\mathrm{K} 250.69 \mathrm{mS} / \mathrm{cm}, \mathrm{pH} 7.3$, water temperature $17.3^{\circ} \mathrm{C}$.

Lake Gregory, south of Halls Creek. Approximate coordinates: $20^{\circ} 12^{\prime}$ S, $127^{\circ} 27^{\prime}$ E. Collected by S. Halse on 29 May 1991 in fresh water (see Halse et al. 1998). One dissected $q$ (nr OS.260), with soft parts in a sealed slide and valves stored dry in micropalaeontological cavity slide (illustrated in Fig. 24I-L).

\section{Brief redescription}

Smallest of the species described here, with females being only slightly longer than $1 \mathrm{~mm}$.

Valves (Fig. 23A-B, E-F) high, with greatest height situated well in front of the middle, dorsal margin evenly sloping towards the posterior side; ventral margin nearly straight. LV (Fig. 23A, E) with anteroventral il of medium size in lower half of valve, antero-dorsal il descending to about halfway along the antero-ventral il. RV (Fig. 23B, F) with antero-ventral lapel almost rectangular, but slightly skewed and bluntly pointed towards the ventral side (Fig. 23C-D, G-H).

Carapace in dorsal and ventral views (Fig. 23K-L) with greatest width in the middle, hirsute and heavily pitted, anteriorly without a rostrum.

Males not yet found in WA. 
Measurements (all measurements in $\mu \mathrm{m}$ - see Table 1 for measurements of all specimens illustrated with SEM)

ㅇ (Pilbara, KIES10, WAMC52357): RV: $\mathrm{L}=1110, \mathrm{H}=676 . \mathrm{LV}: \mathrm{L}=1190, \mathrm{H}=701$.

o (Murchinson/Gascoyne, SIKE9, OC.3351): RV: $\mathrm{L}=1125, \mathrm{H}=672 . \mathrm{LV}: \mathrm{L}=1188, \mathrm{H}=715$.

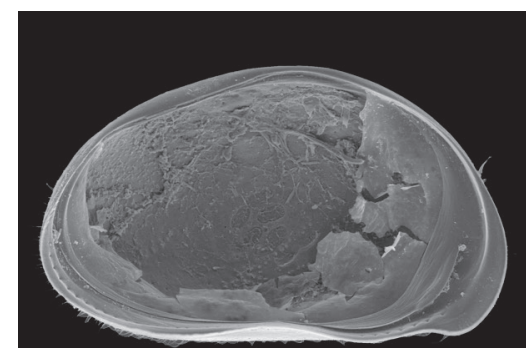

A

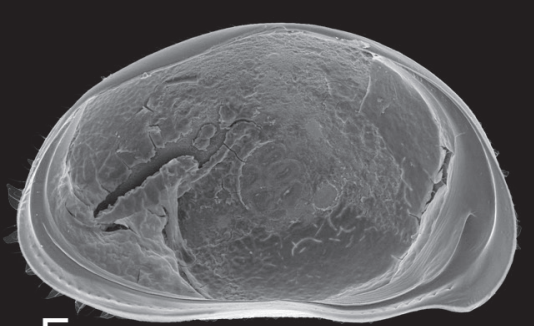

E
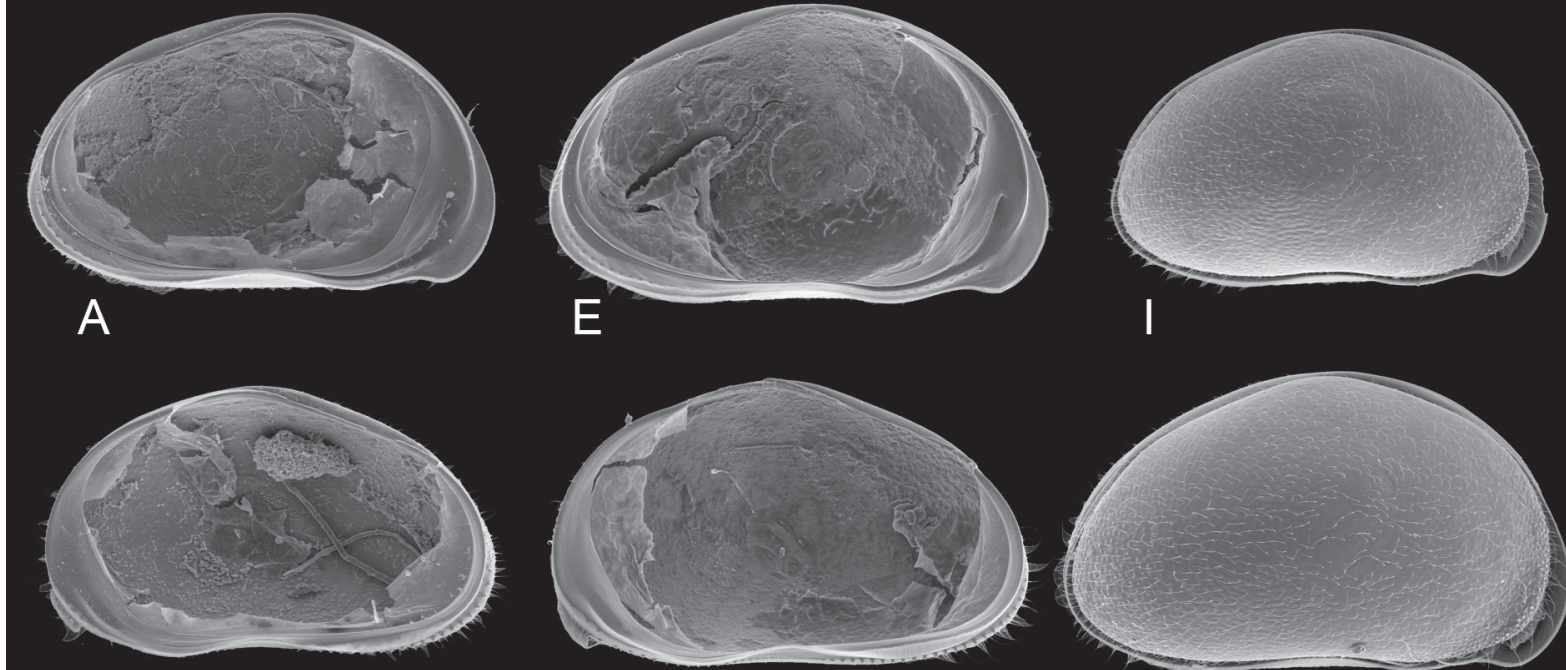

F

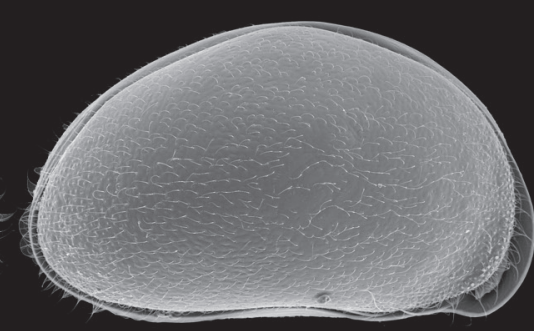

B

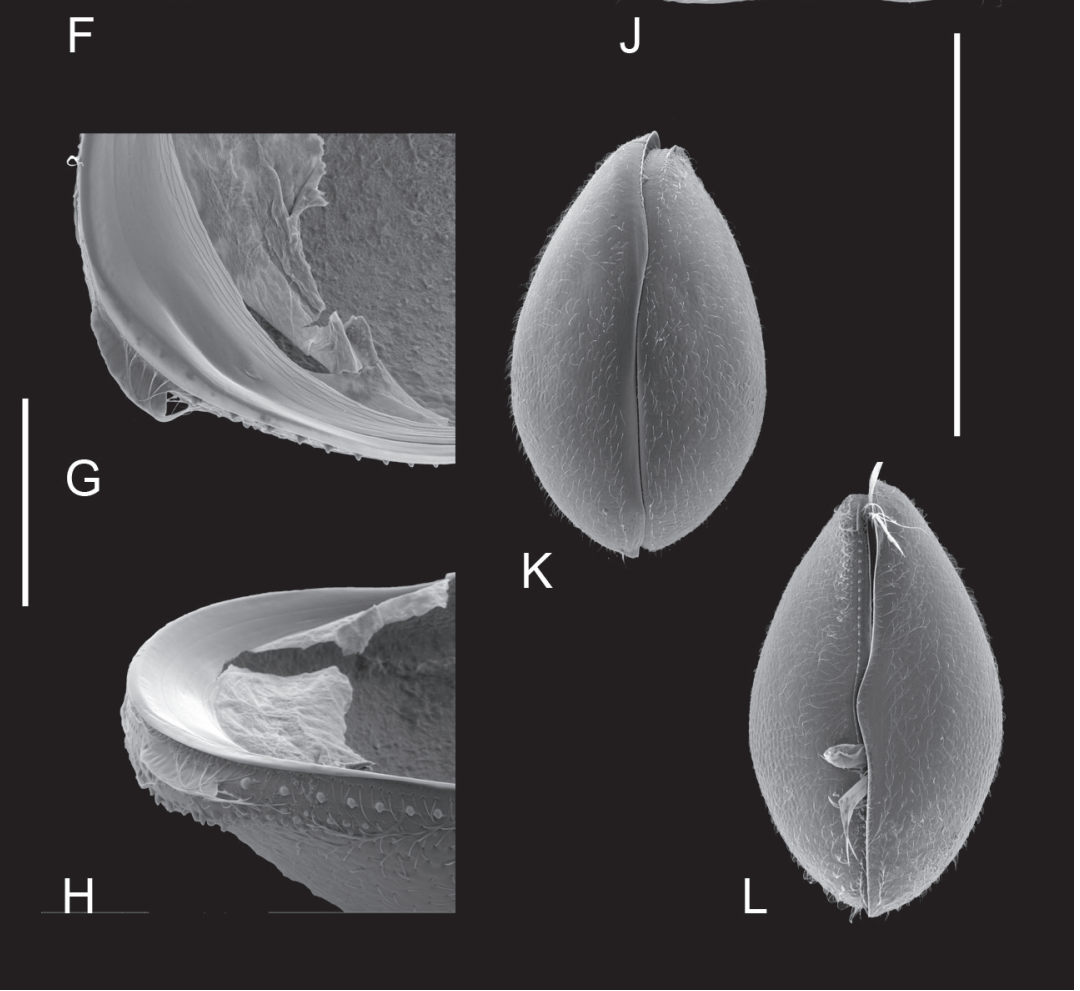

Fig. 23. Bennelongia dedeckkeri, non-type females. - A-D, I, K-L. Dam at Kijlena Well, Pilbara (KIES/10). A. LVi (WAMC52357). B. RVi (idem). C. RVi, detail anterior (idem). D. RVi, detail anterior, slightly tilted (idem). I. CpRL (OC.3350). K. CpD (WAMC52360). L. CpV (WAMC52359). - E-H, J. McNeil Claypan, Murchinson/Gascoyne (SIKE9). E. LVi (OC.3351). F. RVi (idem). G. RVi, detail anterior (idem). H. RVi, detail anterior, tilted (idem). J. CpRL (OC.3352). Scales: A-B, E-F, I-L $=1 \mathrm{~mm}$; C-D, G-H $=200 \mu \mathrm{m}$. 


\section{Ecology and distribution}

Bennelongia dedeckkeri Shearn et al, 2012 was first described from Queensland, from a sexual population. In WA it is not uncommon, but thus far only asexual populations have been found. To date $B$. dedeckkeri is the only species of the B. barangaroo group found in both the eastern and the western parts of Australia.

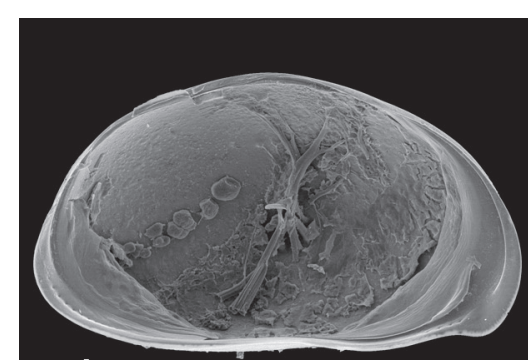

A

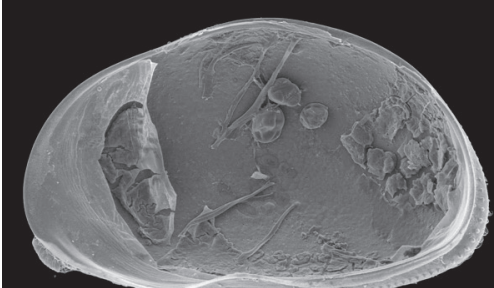

B

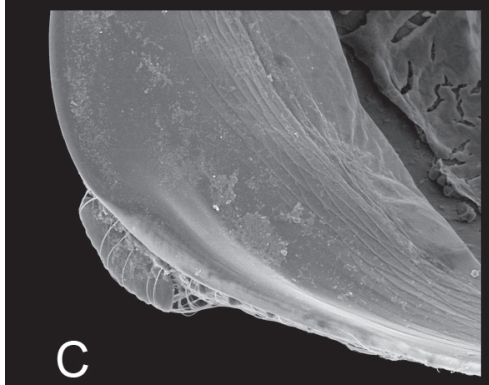

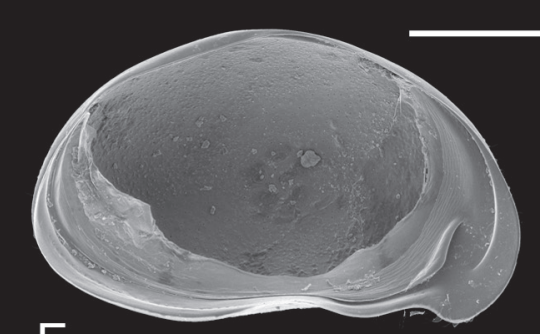

E

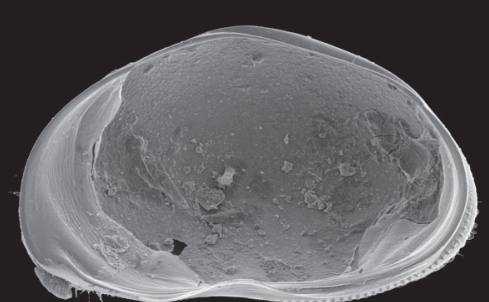

F

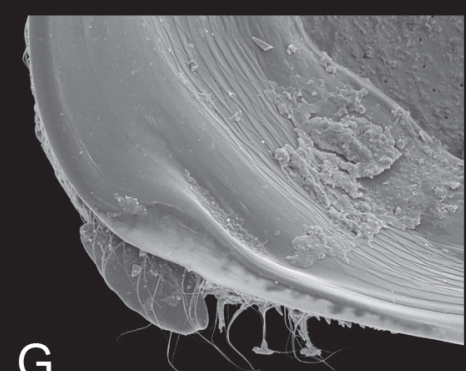

G

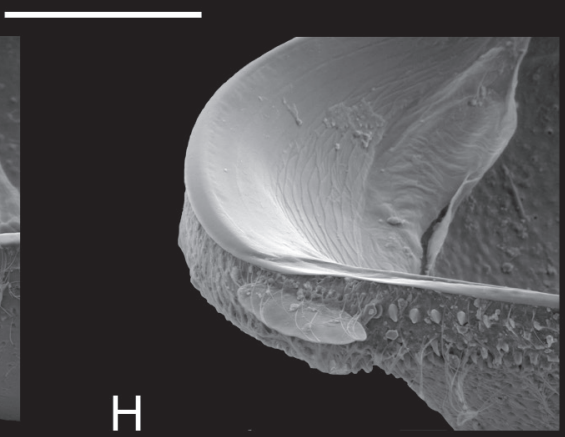

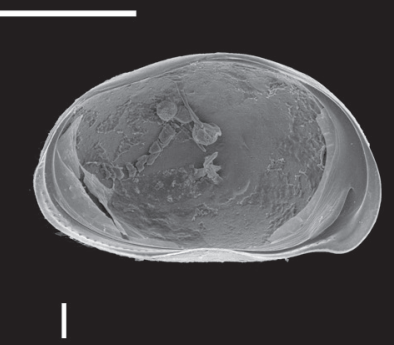

I

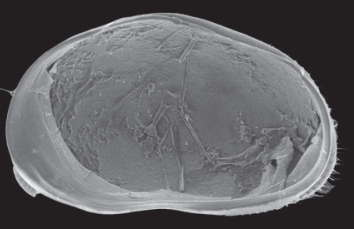

$\mathrm{J}$

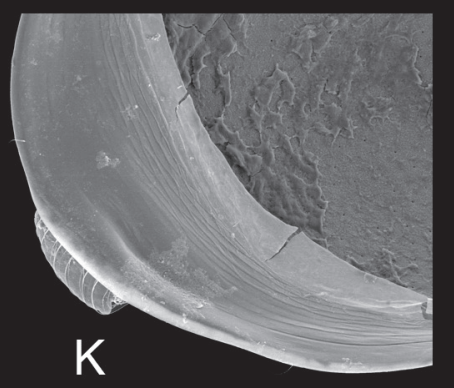

$L$

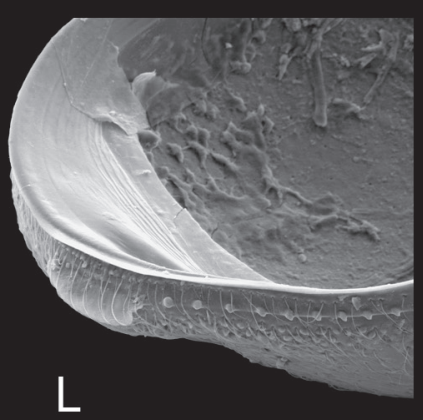

Fig. 24. Bennelongia spp. (no museum nrs), females. - A-D. Bennelongia sp. X1. Crane Pan (OS.255). A. LVi. B. RVi. C. RVi, detail anterior. D. RVi, detail anterior, tilted. - E-H. ? Bennelongia scanloni sp. nov., Lake Cronin (OS.544). E. LVi. F. RVi. G. RVi, detail anterior. H. RVi, detail anterior, tilted. - I-L. Bennelongia dedeckkeri, Lake Gregory (OS.260). I. LVi. J. RVi. K. RVi, detail anterior. L. RVi, detail anterior, tilted. Scales: A-B, E-F, I-J = $1 \mathrm{~mm}$; C-D, G-H, K-L $=200 \mu \mathrm{m}$. 
Bennelongia sp. indet.

There are several single specimens, mostly female, from various (older) collections that could not be identified with certainty. Some of these are here illustrated to allow for future reference, in case new collections will become available. It is entirely possible that some of these specimens represent hybrid clades within the B. barangaroo-group. As none of these specimens have as yet been identified with certainty, no museum numbers have been allocated to them.

\section{Bennelongia sp. X1}

Fig. 24A-D

\section{Material investigated}

One dissected $q$ ( $\mathrm{nr}$ OS.255), with soft parts in a sealed slide and valves stored dry in a micropalaeontological cavity slide.

\section{Locality}

Canegrass covered claypan beside North-West Coastal Highway on Wooramel Station (CB35a), 2540'52” S, 114¹3'14” E, collected by S. Halse and A. Clarke on 24 Aug. 1994.

\section{Morphology and affinities}

The specimen is characterised by (1) a large antero-ventral lapel on the RV, subquadrate and only weakly crenulated, i.e., a shape unlike that of any of the other species (re-) described in the present paper, (2) a relatively small antero-ventral il on the LV and, (3) the bluntly pointed posterior margin of the LV, with a pronounced flange. The latter character is unique within the $B$. barangaroo lineage. If this character is stable and also occurs in other specimens, it could be indicative at a specific level.

\section{Bennelongia sp. X2}

Fig. 25A-D

\section{Material investigated}

One dissected + (nr KMWA.917), with soft parts in a sealed slide and valves stored dry in a micropalaeontological cavity slide.

\section{Locality}

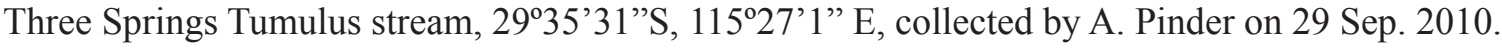

\section{Morphology and affinities}

The specimen has some affinity with $B$. scanloni sp. nov., but the antero-ventral il on the LV is smaller and the antero-ventral lapel of the RV is of a different shape, being larger and almost rectangular.

\section{Remarks}

Several other specimens from the same sample belong to cryptic species E1 of $B$. scanloni sp. nov., as shown by morphological and molecular evidence (see above). 
Bennelongia sp. nov. E2

Fig. 25E-H

\section{Material investigated}

One dissected 9 (nr KMWA.806), with soft parts used for molecular analysis and valves stored dry in a micropalaeontological cavity slide.

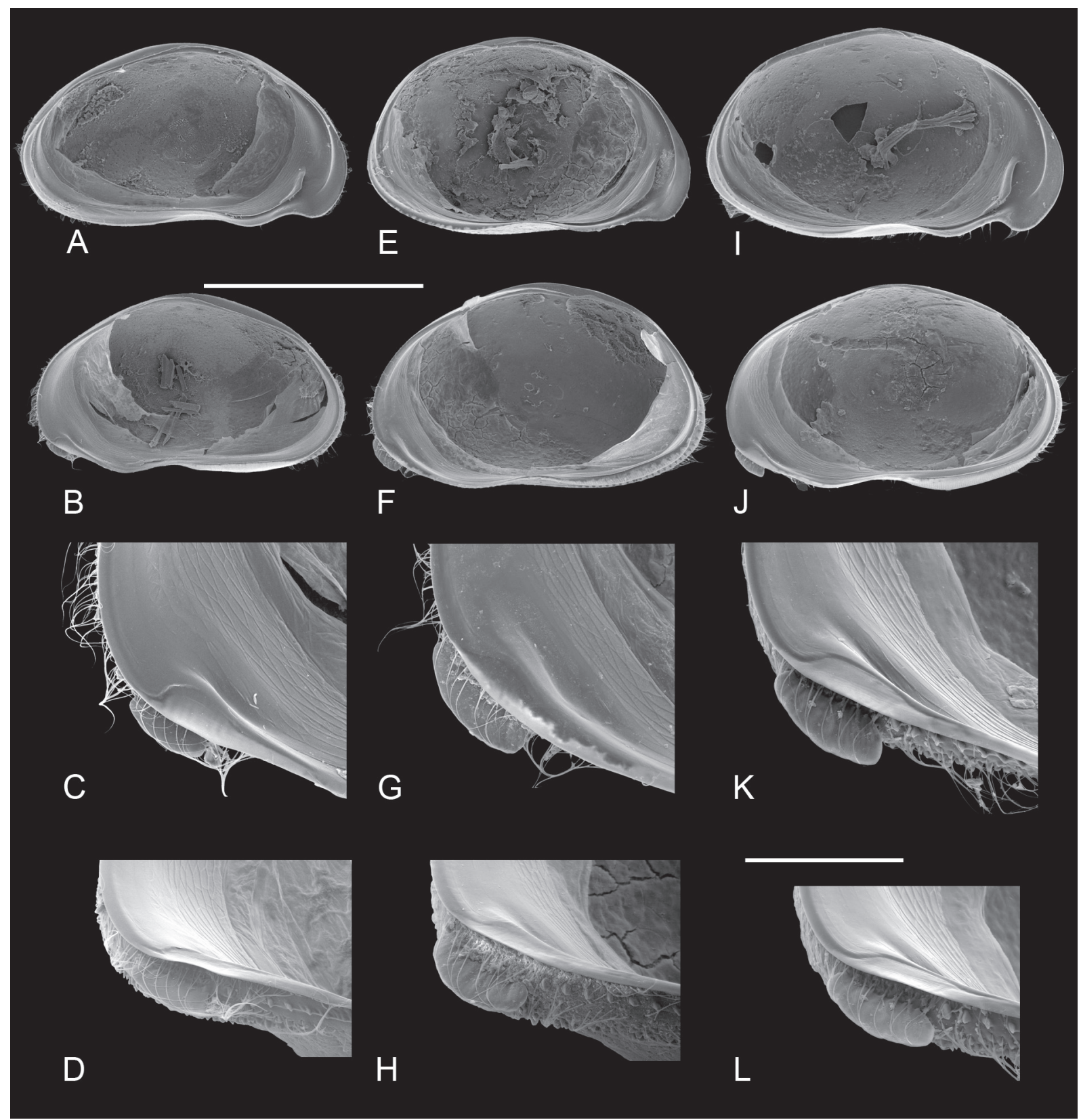

Fig. 25. Bennelongia spp. (no museum nrs). - A-D. Bennelongia sp. X2, Three Springs Tumulus Stream (, KMWA.917). A. LVi. B. RVi. C. RVi, detail anterior. D. RVi, detail anterior, tilted. - E-H. Bennelongia sp. nov. E2, BYK (+, KMWA.806) = Species E2 in Figs 2-3. E. LVi. F. RVi. G. RVi, detail anterior. H. RVi, detail anterior, tilted. - I-L. ? Bennelongia scanloni sp. nov., pool near Esperance (ㅇ, OS.004). I. LVi. J. RVi. K. RVi, detail anterior. L. RVi, detail anterior, tilted. Scales: A-B, E-F, I-J = 1 $\mathrm{mm}$; C-D, G-H, K-L $=200 \mu \mathrm{m}$. 


\section{Locality}

Yakabindie Claypan, approximately $27^{\circ} 34^{\prime} \mathrm{S}, 120^{\circ} 31^{\prime}$ E (sample LN3006), collected by Outback Ecology on 24 Mar. 2011.

\section{Morphology and affinities}

Although general valve appearance again shows some affinities to $B$. scanloni sp. nov., the different shape of the antero-ventral il on the LV (less pronounced and evenly rounded) and the large and subquadrate anteroventral lapel on the RV distinguishes this specimen from all other species (re-)described here. Molecular analysis of the soft parts of the same specimen shows that it clusters outside of the $B$. scanloni sp. nov. group (including cryptic species B2 and E1), and constitutes a different genetic species. Lack of additional material and males prevents us from formally describing this new species here.

\section{General Discussion}

De Deckker (1981a) reported two species of Bennelongia from WA: B. australis (Brady, 1886) and B. barangaroo De Deckker, 1981. Meanwhile, Martens et al. (2012) described nine new species from three separate lineages within this genus from WA (the $B$. australis lineage, the $B$. cygnus lineage and the $B$. pinpi lineage) and showed that $B$. australis is actually a species group with at least seven nominal species and potentially more. Shearn et al. (2012) contributed to the knowledge of the genus Bennelongia in eastern Australia by confirming the validity of B. pinpi De Deckker, 1981 and B. harpago De Deckker $\&$ McKenzie, 1981, using genetics to identify the occurrence of a cryptic lineage within a species of the $B$. australis lineage (B. cuensis Martens et al., 2012), and by describing two new species in the $B$. barangaroo lineage (B. dedeckkeri Shearn et al., 2012 and B. mckenziei Shearn et al., 2012) and one new species within the $B$. nimala lineage (B. regina Shearn et al., 2012). The previous work of De Deckker (1981a,b, 1982) and De Deckker \& McKenzie (1981) as well as these two new papers (Martens et al, 2012; Shearn et al., 2012) show that the genus Bennelongia has extensive radiations in both eastern and western Australia. The present paper formally describes seven new species within the B. barangaroo lineage, redescribes $B$. dedeckkeri and indicates the putative presence of several other species within the B. barangaroo lineage, all from WA. With the seven new species described here, the genus Bennelongia now comprises 25 nominal species (Table 3 ) but several more await formal description.

\section{Morphological features}

Once again, the size and shape, and in some cases the sheer presence or absence, of the antero-ventral lapel on the RV has proven to be indispensable to characterize species of Bennelongia. The plasticity of this feature within the B. barangaroo lineage is amazing and ranges from being fully absent through being small and triangular, to large and drop-like and to a large, elongated and heavily serrated structure. Whereas Martens et al. (2012) hypothesized that the function of the lapel is to lock the sulcus in the LV when valves need to be closed tightly (e.g., when attacked by predators, or in cases where habitat is rapidly desiccating), one could ask why such a wide range of morphologies of the lapel is necessary for highly similar functions in the different lineages and species. When homologous structures have widely different morphologies in closely related species, sexual selection is often invoked as causality. Several authors have indeed already mentioned the potential of sexual selection in ostracod radiations (for example Tsukagoshi 1988; Martens 2000). However, such structures must usually (1) display sexual dimorphism and be most common in males, (2) be accessible during pre-copulation by putative partners, i.e., females, to determine whether or not to accept the male as a partner for reproduction, and (3) occur only in the adult stage, i.e., after the final moult. Only the latter of these three conditions is fulfilled in Bennelongia because there is no apparent sexual dimorphism in lapel-shape and lapels are in general not easily available for inspection by females during the pre-copulatory stage. It is therefore unlikely that lapel morphology has evolved through sexual selection. 
Table 3. Species presently described in Bennelongia and their distribution (species in bold are newly described here). Only certain distributions, based on type localities and documented range extensions, are given here. ${ }^{*}$ indicates the type species.

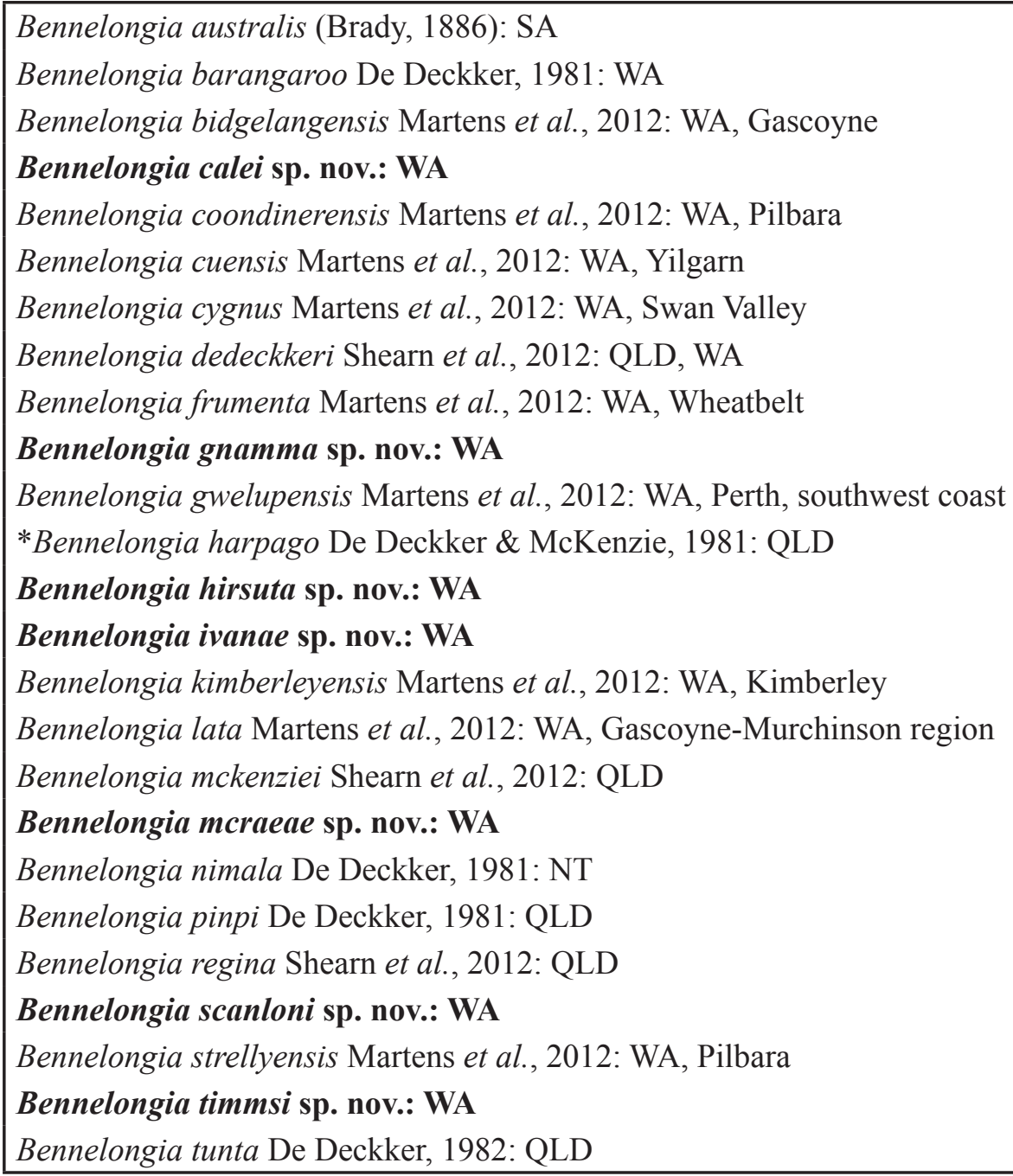

This leaves the possibility that lapels have evolved by chance (not a very parsimonious solution) or that natural selection is acting on the evolution of this morphological feature and that selection pressures are quite stringent. The morphological differences between species living in similar environments (pools, lakes) can be either substantial, as is the case for B. macraeae sp. nov., B. scanloni sp. nov. and B. calei sp. nov., or almost non-existent as in the case of the rock pool dwelling species, which are either cryptic species without morphological differences or have very small differences (B. timmsi sp. nov. with 3 cryptic species and B. gnamma sp. nov.).

\section{Bennelongia timmsi sp. nov. as a biological reality?}

Recent research has shown that cryptic species are not uncommon in non-marine ostracods, as Shearn et al. (2012) found a genetically distinct eastern Australian lineage within the otherwise western Australian B. cuensis Martens et al., 2012, and confirmed that both clades within this species are morphologically indistinguishable. Schön et al. (2012) found several cryptic species within putative ancient asexual darwinulid ostracods, while Bode et al. (2010) revealed no less than 40 cryptic species within the Palaearctic ostracod species Eucypris virens (Jurine, 1820). 
In the $B$. barangaroo lineage, both $B$. timmsi sp. nov. and $B$. scanloni sp. nov. comprise cryptic species as identified by molecular phylogenies based on the mitochondrial COI gene (Fig. 2) and the 4 theta rule (Table 2). Bennelongia scanloni sp. nov. comprises three clusters and three unconnected genetic networks (Fig. 3) of which two are identified as separate genetic species by the 4 theta rule, and the two clusters together form a monophyletic clade within the tree. The smaller TST-clade is phylogenetically slightly separate from the E1 clade, forms an additional network, but does not constitute a separate genetic species. The situation in $B$. scanloni sp. nov. is thus a classic case of a monophyletic species consisting of diverged, but morphologically unrecognisable, clades.

In $B$. timmsi sp. nov., however, the situation is less straightforward. Five clades are recognised in the phylogenetic tree (Fig. 2 - A1-5) and there are six unconnected networks (Fig. 3), of which three are considered valid genetic species (A1, A3, A2+4+5). However, unlike the situation in B. scanloni sp. nov., B. timmsi sp. nov. does not appear as a monophyletic taxon in the phylogenetic analysis, because A1 and A3 cluster together in a different clade than $\mathrm{A} 2+4+5$. Extensive morphological comparisons (Figs 4-11) could not reveal any specific differences in valve or soft part morphology between the clusters (though see below), excluding the possibility that even clades $\mathrm{A} 1+\mathrm{A} 3$ on the one hand and clades $\mathrm{A} 2+4+5$ on the other could be described as different monophyletic species. We considered it of little use to describe two different species when they cannot be identified, except with molecular techniques.

Adding complexity to the phylogenetic uncertainty described above, the morphology of the anteroventral lapel on the RV shows some variability in length and position on the valve among specimens within the $B$. timmsi sp. nov. clades, but this limited variability could not be linked to the phylogenetic position of the specimens. While the shape of the terminal segment of the right prehensile palp was variable within what is assumed to be the same cryptic species (in clade A5), it differed little between cryptic species. Whether or not the differences observed in clade A5 specimens are real or artefacts remains to be seen. The morphologies of the hemipenis-outlines and of the left prehensile palp were fairly constant across all five clades and three cryptic species.

The situation in B timmsi sp. nov. is the first case within the revision of the genus Bennelongia where morphological and molecular data are incongruent. At this stage, we have chosen to follow the results of the morphological analyses, as it seems that, within the tree, the nodes indicated by an * (Fig. 2) are weakly or not statistically supported, and if those nodes are collapsed into a polytomy, the virtual polyphyletic position of B. timmsi sp. nov. would disappear. As soon as we have described and screened all new species of Bennelongia from our collections, the molecular phylogeny of the genus as a whole will be reconstructed and it is hoped that this more complete analysis will shed light on the presumed polyphyletic status of B. timmsi sp. nov. In the meantime, Bennelongia timmsi sp. nov. is proposed here as a valid biological species.

\section{Distribution and Ecology}

The continental-scale distribution of Bennelongia as a whole and phylogeography of selected species will be dealt with elsewhere. At this stage, however, it is useful to point out that almost all species in this genus have fairly restricted distributions: this appears to be so for the species within the B. australis and $B$. cygnus lineages (Martens et al. 2012), as well as in the B. pinpi and the B. barangaroo lineages (Martens et al. loc. cit; Shearn et al. 2012). One notable exception appears to be $B$. dedeckkeri, which has meanwhile been reported from Queensland in eastern Australia (Shearn et al. 2012) and from both northern and central Western Australia (present paper - Fig. 1). Molecular screening has shown that specimens from WA and from QLD even have (near to) identical COI-sequences, showing that this species must have a very efficient means of dispersal compared to its congeners. So far, only parthenogenetic populations have been found in WA, which would be one way to explain a potentially recent expansion from the east (where sexual populations do appear to exist) to the west. Indeed, parthenogens are assumed to be more 
efficient dispersers, as one egg is potentially enough to establish a viable population, whereas sexual groups must have both genders colonising the same habitat, and these moreover must find each other in a spatially and temporally diluted environment (Horne \& Martens 1999). All screened specimens from WA had identical COI sequences (Fig. 3), and the apparent absence of genetic diversity between localities more than $1000 \mathrm{~km}$ apart supports this hypothesis of parthenogens.

Bennelongia dedeckkeri was recovered from both ephemeral lakes and pans (e.g., the McNeil Claypan in Carnarvon), as well as semi-permanent lakes (e.g., the remote Lake Gregory, in the Tanami Desert, but see Halse et al. 1998), and thus seems to be able to thrive in different types of environments. The species might very well have a General Purpose Genotype (Van Doninck et al. 2003). The remainder of the species of the $B$. barangaroo lineage are distributed in more particular habitat types: $B$. timmsi sp. nov., $B$. gnamma sp. nov., $B$. hirsuta sp. nov. and $B$. ivanae sp. nov. occur only in rock pools, and this diversity within a single lineage confirms the rock pools on the granite outcrops in southwestern Australia as foci of diversification of aquatic animals (Pinder et al. 2000). Bennelongia mcraeae sp. nov. and B. scanloni sp. nov. were sampled in seasonal or episodic soft-sediment lakes and pools only. Bennelongia calei sp. nov. occurs mainly in the latter types of habitats, but was also found in at least two localities in pit-gnammas, which are deep and narrow rock pools. Populations from both types of habitats were also shown to belong to one and the same genetic species (Fig. 2), and this species thus has a puzzling autecology.

\section{Acknowledgements}

The authors gratefully acknowledge the financial support by an ABRS-grant (nr RF211-33: 'Biodiversity and taxonomy of Ostracoda (Crustacea) from temporary water bodies of inland Western Australia') and by an Edith Cowan University Industry Collaboration grant. KM \& IS acknowledge the Western Australian Department of Parks and Wildlife (2006) and Bennelongia Pty Ltd (2008, 2009, 2010) for financial support during several scientific stays in Perth, as well as the financial contribution of the FWO Vlaanderen (Fund for Scientific Research, Flanders) in the form of travel grants in 2010 (V4.172.10N \& V4.173.10N) and the projects 1.5.172.09 (Krediet aan Navorsers) and G.0118.03N (projectonderzoek). KM and IS also thank the people of Bennelongia Pty Ltd (Perth, WA) for continuous logistic support (lab space, use of microscopes, etc.) and for unfailing companionship in the lab and their son Emrys for invaluable help with sorting living specimens in the field as well as in the lab. David J. Cale, A. Clarke and Adrian Pinder (DEC, Woodvale, Australia), Brian V. Timms (Newcastle, Australia), Outback Ecology (Perth, Australia) and Jane McRae (Bennelongia Pty Ltd, Perth, Australia) are acknowledged for collecting most of the material described in the present paper.

Julien Cilis and Claudine Behen (RBINS, Brussels, Belgium) provided technical assistance with the SEM micrographs and with the line drawings, respectively. Kristiaan Hoedemakers (RBINS, Brussels, Belgium) produced the SEM plates and supervised the entire production of the manuscript. Mike Scanlon (Bennelongia Pty Ltd, Perth, WA) produced the map. IS thanks Annette Koenders (Edith Cowan University, Perth) and Mike Johnson (University of Western Australia, Perth) for providing laboratory space for the molecular part of this research. Dr Finn Viehberg (Köln, Germany) and Dr David J. Horne (London, U.K.) are thanked for their valuable comments as referees.

\section{References}

Altschul S.F., Gish W., Miller W., Myers E.W. \& Lipman D.J. 1990. Basic local alignment search tool. Journal of Molecular Biology 215: 403-410. http://dx.doi.org/10.1016/S0022-2836(05)80360-2

Birky Jr. C.W. 2011. Species detection and identification in sexual organisms using population genetic theory and DNA sequences. PLoS ONE 8: e52544. http://dx.doi.org/10.1371/journal.pone.0052544 
Birky Jr. C.W. \& Barraclough T.G. 2009. Asexual speciation. In: Schön I., Martens K. \& van Dijk P. (eds) Lost Sex. Springer Scientific Publishers, Dordrecht: 201-216.

Birky Jr. C.W., Adams J., Gemmel M. \& Perry J. 2010. Using Population Genetic Theory and DNA sequences for species detection and identification in asexual organisms. PLoS ONE 5: e10609. http:// dx.doi.org/10.1371/journal.pone.0010609

Birky Jr. C.W., Ricci C., Melone G. \& Fontaneto D. 2011. Integrating DNA and morphological taxonomy to describe diversity in poorly studied microscopic animals: new species of the genus Abrochtha Bryce, 1910 (Rotifera: Bdelloidea: Philodinavidae). Zoological Journal of the Linnean Society 161: 723-734. http://dx.doi.org/10.1111/j.1096-3642.2010.00674.x

Bode S.N.S., Lamatsch D.K., Martins M.J.F., Schmit O., Vandekerkhove J., Mezquita F., Namiotko T., Rossetti G., Schön I., Butlin R.K. \& Martens K. 2010. Exceptional cryptic diversity and multiple origins of parthenogenesis in a freshwater ostracod. Molecular Phylogeny and Evolution 54: 542-552. http:// dx.doi.org/10.1016/j.ympev.2009.08.022

Broodbakker N.W. \& Danielopol D.L. 1982. The chaetotaxy of Cypridacea (Crustacea, Ostracoda) limbs: proposals for a descriptive model. Bijdragen tot de Dierkunde 52: 103-120.

Cale D.J., Halse S.A. \& Walker C.D. 2004. Wetland monitoring in the wheatbelt of south-west Western Australia: site descriptions, waterbird, aquatic invertebrate and groundwater data. Conservation Science Western Australia 5: 20-135.

Clement M., Posada D. \& Crandall K. 2000. TCS: a computer program to estimate gene genealogies. Molecular Ecology 9: 1657-1660. http://dx.doi.org/10.1046/j.1365-294x.2000.01020.x

Darriba D., Taboada G.L., Doallo R. \& Posada D. 2012. jModelTest 2: more models, new heuristics and parallel computing. Nature Methods 9: 772. http://dx.doi.org/10.1038/nmeth.2109

De Deckker P. 1981a. Taxonomy and ecology notes of some ostracods from Australian inland waters. Transactions of the Royal Society of South Australia 105 (3): 91-138.

De Deckker P. 1981b. Ostracoda from Australian inland waters - notes on taxonomy and ecology. Transactions of the Royal Society of Victoria 93 (1): 43-85.

De Deckker P. 1982. On Bennelongia tunta De Deckker sp. nov. A Stereo-Atlas of Ostracod Shells 9 (21): 117-124.

De Deckker P. \& McKenzie K.G. 1981. Bennelongia, a new cyprididid ostracod genus from Australasia. Transactions of the Royal Society of South Australia 105 (2): 53-58.

De Deckker P. \& Martens K. 2013. Extraordinary morphological changes in valve morphology during the ontogeny of several species of the Australian ostracod genus Bennelongia (Crustacea, Ostracoda). European Journal of Taxonomy 36: 1-37. http://dx.doi.org/10.5852/ejt.2013.36

Folmer O., Black M., Hoeh W., Lutz R. \& Vrijenhoek R. 1994. DNA primers for amplification of mitochondrial cytochrome c oxidase subunit I from diverse metazoan invertebrates. Molecular Marine Biology and Biotechnology 3: 294-299.

Fontaneto D., Herniou E.A., Boschetti C., Caprioli M., Melone G., Ricci C. \& Barraclough T.G. 2007. Independently evolving species in asexual bdelloid rotifers. PLoS ONE 5: e87. http://dx.doi.org/10.1371/ journal.pbio.0050087

Fontaneto D., Kaya M., Herniou E.A. \& Barraclough T.G. 2009. Extreme levels of hidden diversity in microscopic animals (Rotifera) revealed by DNA taxonomy. Molecular Phylogenetics and Evolution 53: 182-189. http://dx.doi.org/10.1016/j.ympev.2009.04.011 
Guindon S. \& Gascuel O. 2003. PhyML - a simple, fast, and accurate algorithm to estimate large phylogenies by maximum likelihood. Systematic Biology 52: 696-704. http://dx.doi. org/10.1080/10635150390235520

Hall T. 2007. BioEdit: Biological sequence alignment editor for Win95/98/NT/2K/XP [Online]. Website last modified on June 27, 2007 (accessed on September 13, 2011). Available at http://www.mbio.ncsu. edu/BioEdit/bioedit.html

Halse S.A. 2002. Diversity of Ostracoda (Crustacea) in inland waters of Western Australia. Verhandlungen der Internationalen Vereinigung für theoretische und angewandte Limnologie 28: 914-918.

Halse S.A., Shiel R.J. \& Williams W.D. 1998. Aquatic invertebrates of Lake Gregory, north-western Australia, in relation to salinity and ionic composition. Hydrobiologia 381: 15-29. http://dx.doi. org/10.1023/A:1003263105122

Horne D.J. \& Martens K. 1999. Geographical parthenogenesis in European non-marine ostracods: postglacial invasion or Holocene stability? Hydrobiologia 391: 1-7. http://dx.doi.org/10.1023/ A:1003508210166

Horne D.J., Cohen A. \& Martens K. 2002. Taxonomy, Morphology and Biology of Quaternary and Living Ostracoda. In: Holmes J.A. \& Chivas A.R. (eds) The Ostracoda: Application in Quaternary Research. Geophysical Monograph 131. American Geophysical Union, Washington, DC: 5-36.

Larkin M.A., Blackshields G., Brown N.P., Chenna R., McGettigan P.A., McWilliam H., Valentin F., Wallace I.M., Wilm A., Lopez R., Thompson J.D., Gibson T.J. \& Higgins D.G. 2007. Clustal W and Clustal X version 2.0. Bioinformatics 23: 2947-2948. http://dx.doi.org/10.1093/bioinformatics/btm404

Martens K. 1987. Homology and functional morphology of the sexual dimorphism in the antenna of Sclerocypris Sars, 1924 (Crustacea, Ostracoda, Megalocypridinae). Bijdragen tot de Dierkunde 57: 183190.

Martens K. 2000. Factors affecting the divergence of mate recognition systems in the Limnocytherinae (Crustacea, Ostracoda ). In: Horne D.J. \& Martens K. (eds) Proceedings of the XIII International Symposium on Ostracoda. Hydrobiologia 419: 83-101. http://dx.doi.org/10.1023/A:1003954513004

Martens K., Halse S. \& Schön I. 2012. Nine new species of Bennelongia De Deckker \& McKenzie, 1981 (Crustacea, Ostracoda) from Western Australia, with the description of a new subfamily. European Journal of Taxonomy 8: 1-56. http://dx.doi.org/10.5852/ejt.2012.8

Pinder A.M., Halse S.A., Shiel R.J. \& McRae J.M. 2000. Granite outcrop pools in south-western Australia: foci of diversification and refugia for aquatic invertebrates. Journal of the Royal Society of Western Australia 83: 149-161.

Ronquist F., Teslenko M., van der Mark P., Ayres D., Darling A., Höhna S., Larget B., Liu L., Suchard M.A. \& Huelsenbeck J.P. 2012. MrBayes 3.2: Efficient Bayesian phylogenetic inference and model choice across a large model space. Systematic Biology 61: 539-542. http://dx.doi.org/10.1093/sysbio/ sys029

Schön I., Pinto R.L., Halse S.A., Smith A.J., Martens K. \& Birky Jr. C.W. 2012. Cryptic species in putative ancient asexual darwinulids (Crustacea, Ostracoda). PLOS ONE 7: e39844. http://dx.doi. org/10.1371/journal.pone.0039844

Shearn R., Koenders A., Halse S., Schön I. \& Martens K. 2012. A review of Bennelongia De Deckker \& Mckenzie, 1981 (Crustacea, Ostracoda) species from eastern Australia, with the description of three new species. European Journal of Taxonomy 25: 1-35. http://dx.doi.org/10.5852/ejt.2012.25 
Tamura K., Peterson D., Peterson N., Stecher G., Nei M. \& Kumar S. 2011. MEGA5: Molecular Evolutionary Genetics Analysis using Likelihood, Distance, and Parsimony methods. Molecular Biology and Evolution 28: 2731-2739. http://dx.doi.org/10.1093/molbev/msr121

Timms B.V. 2002. The fairy shrimp Branchinella Sayce (Crustacea: Anostraca: Thamnocephalidae) in Western Australia, including a description of four new species. Hydrobiologia 486: 71-89. http://dx. doi. org/10.1023/A:1021330230369

Tsukagoshi A. 1988. Reproductive character displacement in the ostracod genus Cythere. Journal of Crustacean Biology 8: 563-575. http://dx.doi.org/10.2307/1548693

Van Doninck K., Schön I., Maes F., De Bruyn L. \& Martens K. 2003. Ecological strategies in the ancient asexual animal group Darwinulidae (Crustacea, Ostracoda). Freshwater Biology 48: 1285-1294. http:// dx.doi.org/10.1046/j.1365-2427.2003.01078.x

Manuscript received: 29 July 2013

Manuscript accepted: 9 October 2013

Published on: 25 November 2013

Topic editor: Rudy Jocqué

Desk editor: Kristiaan Hoedemakers

Printed versions of all papers are also deposited in the libraries of the institutes that are members of the EJT consortium: Muséum National d'Histoire Naturelle, Paris, France; National Botanic Garden of Belgium, Meise, Belgium; Royal Museum for Central Africa, Tervuren, Belgium; Natural History Museum, London, United Kingdom; Royal Belgian Institute of Natural Sciences, Brussels, Belgium; Natural History Museum of Denmark, Copenhagen, Denmark. 